\title{
A necklace of dense cores in the high-mass star forming region G35.20-0.74 N: ALMA observations ${ }^{\star}, \star \star$
}

\author{
Á. Sánchez-Monge ${ }^{1,2}$, M. T. Beltrán ${ }^{2}$, R. Cesaroni ${ }^{2}$, S. Etoka ${ }^{3,4}$, D. Galli ${ }^{2}$, M. S. N. Kumar ${ }^{5}$, L. Moscadelli ${ }^{2}$, \\ T. Stanke ${ }^{6}$, F. F. S. van der Tak ${ }^{7,8}$, S. Vig ${ }^{9}$, C. M. Walmsley ${ }^{2,10}$, K.-S. Wang ${ }^{11}$, H. Zinnecker ${ }^{12}$, D. Elia ${ }^{13}$, \\ S. Molinari ${ }^{13}$, and E. Schisano ${ }^{14}$ \\ ${ }^{1}$ I. Physikalisches Institut, Universität zu Köln, Zülpicher Str. 77, 50937 Köln, Germany \\ e-mail: sanchez@ph1.uni-koeln.de \\ 2 Osservatorio Astrofisico di Arcetri, INAF, Largo Enrico Fermi 5, 50125 Firenze, Italy \\ 3 Hamburger Sternwarte, Gojenbergsweg 112, 21029 Hamburg, Germany \\ 4 Jodrell Bank Centre for Astrophysics, School of Physics and Astronomy, University of Manchester, Manchester M13 9PL, UK \\ 5 Centro de Astrofísica da Universidade do Porto, rua das Estrelas, 4150-762 Porto, Portugal \\ ${ }^{6}$ ESO, Karl-Schwarzschilk-Strasse 2, 85748 Garching bei München, Germany \\ 7 SRON Netherlands Institute for Space Research, PO Box 800, 9700 AV Groningen, The Netherlands \\ 8 Kapteyn Astronomical Institute, University of Groningen, 9700 AV Groningen, The Netherlands \\ 9 Dpt. of Earth and Space Science, Indian Institute of Space Science and Technology, Thiruvananthapuram, 695547 Kerala, India \\ 10 Dublin Institute for Advanced Studies (DIAS), 31 Fitzwilliam Place, 2 Dublin, Ireland \\ 11 Leiden Observatory, Leiden University, PO Box 9513, 2300 RA Leiden, The Netherlands \\ 12 SOFIA Science Center, NASA Ames Research Center, Mailstop 232-12, Moffett Field, CA 94035, USA \\ 13 Istituto di Astrofisica e Planetologia Spaziali (IAPS-INAF), via Fosso del Cavaliere 100, 00133 Roma, Italy \\ 14 Infrared Processing and Analysis Center, Institute of Technology, Pasadena, CA 91125, USA
}

Received 18 April 2014 / Accepted 9 June 2014

\section{ABSTRACT}

Context. The formation process of high-mass stars (with masses $>8 M_{\odot}$ ) is still poorly understood, and represents a challenge from both the theoretical and observational points of view. The advent of the Atacama Large Millimeter Array (ALMA) is expected to provide observational evidence to better constrain the theoretical scenarios.

Aims. The present study aims at characterizing the high-mass star forming region G35.20-0.74 N, which is found associated with at least one massive outflow and contains multiple dense cores, one of them recently found associated with a Keplerian rotating disk. Methods. We used the radio-interferometer ALMA to observe the G35.20-0.74 N region in the submillimeter continuum and line emission at $350 \mathrm{GHz}$. The observed frequency range covers tracers of dense gas (e.g., $\mathrm{H}^{13} \mathrm{CO}^{+}, \mathrm{C}^{17} \mathrm{O}$ ), molecular outflows (e.g., SiO), and hot cores (e.g., $\mathrm{CH}_{3} \mathrm{CN}, \mathrm{CH}_{3} \mathrm{OH}$ ). These observations were complemented with infrared and centimeter data.

Results. The ALMA $870 \mu$ m continuum emission map reveals an elongated dust structure $(\sim 0.15 \mathrm{pc}$ long and $\sim 0.013 \mathrm{pc}$ wide; full width at half maximum) perpendicular to the large-scale molecular outflow detected in the region, and fragmented into a number of cores with masses $\sim 1-10 M_{\odot}$ and sizes $\sim 1600$ AU (spatial resolution $\sim 960 \mathrm{AU}$ ). The cores appear regularly spaced with a separation of $\sim 0.023 \mathrm{pc}$. The emission of dense gas tracers such as $\mathrm{H}^{13} \mathrm{CO}^{+}$or $\mathrm{C}^{17} \mathrm{O}$ is extended and coincident with the dust elongated structure. The three strongest dust cores show emission of complex organic molecules characteristic of hot cores, with temperatures around $200 \mathrm{~K}$, and relative abundances $0.2-2 \times 10^{-8}$ for $\mathrm{CH}_{3} \mathrm{CN}$ and $0.6-5 \times 10^{-6}$ for $\mathrm{CH}_{3} \mathrm{OH}$. The two cores with highest mass (cores A and B) show coherent velocity fields, with gradients almost aligned with the dust elongated structure. Those velocity gradients are consistent with Keplerian disks rotating about central masses of 4-18 $M_{\odot}$. Perpendicular to the velocity gradients we have identified a large-scale precessing jet/outflow associated with core B, and hints of an east-west jet/outflow associated with core A.

Conclusions. The elongated dust structure in G35.20-0.74 N is fragmented into a number of dense cores that may form high-mass stars. Based on the velocity field of the dense gas, the orientation of the magnetic field, and the regularly spaced fragmentation, we interpret this elongated structure as the densest part of a 1D filament fragmenting and forming high-mass stars.

Key words. stars: formation - stars: massive - ISM: individual objects: G35.20-0.74 N - ISM: molecules ISM: jets and outflows

\section{Introduction}

High-mass stars (O- and B-type stars with masses $\gtrsim 8 M_{\odot}$ ) are crucial for the understanding of many physical phenomena in the Galaxy; however, their first stages of formation are still poorly

\footnotetext{
* Appendix is available in electronic form at http: //www . aanda.org

$\star \star$ Reduced FITS files (cube and continum) are only available at the CDS via anonymous ftp to cdsarc.u-strasbg. fr (130.79.128.5) or via

http://cdsarc.u-strasbg.fr/viz-bin/qcat? J/A+A/569/A11
}

understood and represent a challenge from both a theoretical and observational point of view. A number of different theoretical scenarios have been proposed to explain the formation of OB-type stars (see Zinnecker \& Yorke 2007 and Tan et al. 2014 for a review). Even with their differences, the most accepted theories (monolithic collapse in a turbulence-dominated core - Krumholz et al. 2009; competitive accretion driven by a stellar cluster - Bonnell \& Bate 2006; Bondi-Hoyle accretion - Keto 2007) agree in the prediction of the formation of circumstellar disks around stars of all masses. However, while in the low-mass regime, circumstellar disks have been extensively 
identified and studied (e.g., Simon et al. 2000), there are only a few convincing disks (with sizes $\leq 1000 \mathrm{AU}$ ) found around B-type protostars (e.g., Schreyer et al. 2002; Cesaroni et al. 2005, 2007; Carrasco-González et al. 2012; Wang et al. 2012). Moreover, convincing evidence of centrifugally supported disks around O-type protostars still remains elusive. Infrared interferometric observations of the $\mathrm{CO}$ overtone bands suggest that disks might be associated with O-type stars (e.g., Kraus et al. 2010; de Wit et al. 2011; Boley et al. 2013), but (sub)millimeter images have only revealed the existence of huge $(\sim 0.1 \mathrm{pc})$ and massive $\left(\sim 100 M_{\odot}\right)$ cores undergoing solid-body rotation, the so-called "toroids" (e.g., Beltrán et al. 2011). The detection of circumstellar disks, with expected sizes $\leq 1000$ AU (e.g., Cesaroni et al. 2007), requires angular resolutions < 0.'1 (assuming a typical distance of $5 \mathrm{kpc}$ ). Thus, the lack of observed disks is expected to dramatically change with the advent of the Atacama Large Millimeter Array (ALMA), with which resolutions 0.' 1 will be easily obtained.

In a progressive effort to confront the theoretical scenario of the formation of massive stars through disk-mediated accretion, and to set the stage for a follow-up quest for the more challenging disks around O-type stars, we carried out ALMA Cycle 0 observations towards two IR disk candidates around B-type protostars in G35.20-0.74N and G35.03+0.35. Results on $\mathrm{G} 35.03+0.35$ are presented in a parallel paper by Beltrán et al. (2014), while first results on G35.20-0.74 N have been already published in Sánchez-Monge et al. (2013b). In this work, we present the ALMA results on G35.20-0.74 N in a more extensive and detailed way.

G35.20-0.74 is a star forming complex located at a distance of $2.19_{-0.20}^{+0.24} \mathrm{kpc}$ (Zhang et al. 2009). The main site of highmass star formation activity, known as G35.20-0.74 N (hereafter G35.20N), is associated with the IRAS source $18556+0136$ and has a bolometric luminosity $\sim 1-10 \times 10^{4} L_{\odot}$ (e.g., Gibb et al. 2003; Sánchez-Monge et al. 2013b; Zhang et al. 2013). In the infrared, the region is dominated by the presence of a butterflyshaped nebula oriented NE-SW (see Fig. 1a). This direction is coincident with the orientation $\left(\mathrm{PA} \approx 58^{\circ}\right.$ ) of a bipolar outflow observed in ${ }^{12} \mathrm{CO}$ (e.g., Dent et al. 1985a; Gibb et al. 2003; Birks et al. 2006; López-Sepulcre et al. 2009; Qiu et al. 2013). The ${ }^{12} \mathrm{CO}(1-0)$ line emission appears to trace also a N-S collimated flow (see Birks et al. 2006), coinciding with a thermal radio jet (e.g., Heaton \& Little 1988; Gibb et al. 2003) seen also at IR wavelengths (e.g., Dent et al. 1985b; Walther et al. 1990; Fuller et al. 2001; De Buizer 2006; Zhang et al. 2013). The different orientations between the poorly collimated NE-SW outflow and the $\mathrm{N}-\mathrm{S}$ jet have been interpreted as manifestations of the same flow undergoing precession (e.g., Little et al. 1998; Gibb et al. 2003), or as emission coming from a number of flows driven by the different sources forming in the G35.20N complex (e.g., Gibb et al. 2003; Birks et al. 2006).

Emission from dense gas tracers (e.g., $\mathrm{NH}_{3}, \mathrm{C}^{18} \mathrm{O}, \mathrm{CS}, \mathrm{HCN}$, $\mathrm{H}^{13} \mathrm{CO}^{+}$), as well as continuum dust emission at millimeter wavelengths (e.g., Little et al. 1985; Brebner et al. 1987; Gibb et al. 2003; López-Sepulcre et al. 2009), have revealed the presence of a dense clump elongated perpendicular to the NE-SW outflow, with a velocity gradient from NW $\left(\sim 32 \mathrm{~km} \mathrm{~s}^{-1}\right)$ to SE $\left(\sim 36 \mathrm{~km} \mathrm{~s}^{-1}\right)$. This velocity gradient was first thought to originate from a large $(\sim 0.6 \mathrm{pc})$ flattened structure rotating about the axis of the NE-SW outflow. Subsequently, on the basis of their $\mathrm{H}^{13} \mathrm{CO}^{+}$and $\mathrm{H}^{13} \mathrm{CN}$ observations, Gibb et al. (2003) propose that this is actually a fragmented rotating envelope containing multiple young stellar objects. The high angular resolution map at $870 \mu \mathrm{m}$ obtained with ALMA (Sánchez-Monge et al. 2013b) reveals an elongated structure containing a chain of compact cores (see Fig. 1b), and confirms a significant level of clumpiness. The two strongest cores (named core A and core B in Sánchez-Monge et al. 2013b) are found close to the center of the elongated structure (core A to the NW and core B to the $\mathrm{SE}$, separated by $\sim 2^{\prime \prime}$ ). These cores, detected in several complex organic molecules, show velocity gradients roughly aligned with the elongated structure, i.e., perpendicular to the large-scale NE-SW outflow, but opposite to each other. The kinematics of core $\mathrm{B}$, which is located at the center of symmetry of the outflow/jet, have been modeled as a Keplerian disk rotating about a central mass of $\sim 18 M_{\odot}$ (Sánchez-Monge et al. 2013b).

Finally, emission from different maser species $\left(\mathrm{H}_{2} \mathrm{O}\right.$, $\left.\mathrm{OH}, \mathrm{CH}_{3} \mathrm{OH}\right)$ has been detected toward the $\mathrm{G} 35.20 \mathrm{~N}$ region (e.g., Brebner et al. 1987; Forster \& Caswell 1989, 1999; Hutawarakorn \& Cohen 1999; Vlemmings 2008; Sugiyama et al. 2008; Surcis et al. 2012). The highest angular-resolution observations show two main regions for the maser emission, separated by about 2.'3 ( 5000 AU), and oriented SE-NW. Hutawarakorn \& Cohen (1999) measured magnetic field strengths (through $1665 \mathrm{MHz} \mathrm{OH}$ Zeeman-splitting observations) between $-2.5 \mathrm{mG}$ to the $\mathrm{NW}$, and $+5.2 \mathrm{mG}$ to the SE. Polarimetric observations of $6.7 \mathrm{GHz} \mathrm{CH}_{3} \mathrm{OH}$ masers (Surcis et al. 2012), indicate strong (5-12\%) linear polarization, with the magnetic field oriented almost E-W, PA $\approx 80$ $110^{\circ}$. Recent polarimetric dust observations carried out with the SMA (Qiu et al. 2013), revealed that the magnetic field is aligned with the long axis of the elongated dust structure to the $\mathrm{NW}$ roughly following the large-scale field $\left(\mathrm{PA} \approx 56^{\circ}\right.$; Vallée \& Bastien 2000), but changes its direction drastically by approximately $90^{\circ}$ when moving to the south of the filament (at a position between cores B and C; see Fig. 2 in Qiu et al. 2013). The field strength is estimated to be $\sim 1 \mathrm{mG}$, i.e., consistent with the strength measured via Zeeman-splitting observations (Hutawarakorn \& Cohen 1999).

Given the complexity of this region and the (sometimes contradictory) findings of previous observations, the main goal of our ALMA observations was to address some fundamental questions such as the fragmentation level of the elongated structure, the kinematics of the dense gas at different scales and the number of outflows and powering sources. This paper is organized as follows. In Sect. 2, we describe our ALMA observations. In Sect. 3, we present the main results and reconstruct the spectral energy distribution of $\mathrm{G} 35.20 \mathrm{~N}$ from the millimeter to the near IR. In Sect. 4, we analyze and discuss our findings focusing on the kinematical properties of the structures detected. Finally, in Sect. 5 we summarize the main results and draw the conclusions.

\section{ALMA observations}

G35.20N was observed with ALMA in Cycle 0 between May and June 2012. The source was observed in Band 7 $(\sim 350 \mathrm{GHz})$ with the 16 antennas of the array in the extended configuration, i.e., baselines in the range $36-400 \mathrm{~m}$, providing sensitivity to structures $\lesssim 2$ ". The digital correlator was configured in four spectral windows (with dual polarization) of $1875 \mathrm{MHz}$ and 3840 channels each, providing a resolution of $\sim 0.4 \mathrm{~km} \mathrm{~s}^{-1}$. The four spectral windows covered the frequency ranges [336849.57-338723.83] $\mathrm{MHz}$, [334 965.73-336839.99] MHz, [348 843.78-350718.05] MHz, and [346891.29-348765.56] MHz. The phase center of the observations is $\alpha(\mathrm{J} 2000)=18^{\mathrm{h}} 58^{\mathrm{m}} 13^{\mathrm{s}} .03, \delta(\mathrm{J} 2000)=01^{\circ} 40^{\prime} 36^{\prime \prime} 0$. Flux, gain, and bandpass calibrations were obtained through observations of Neptune and J1751+096. The data were calibrated 


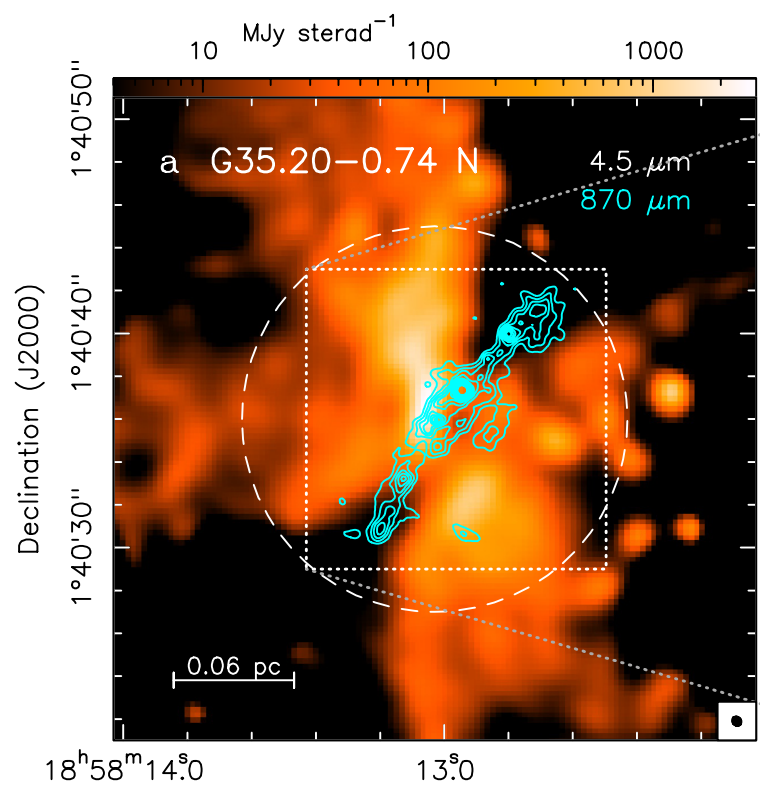

Right Ascension (J2000)

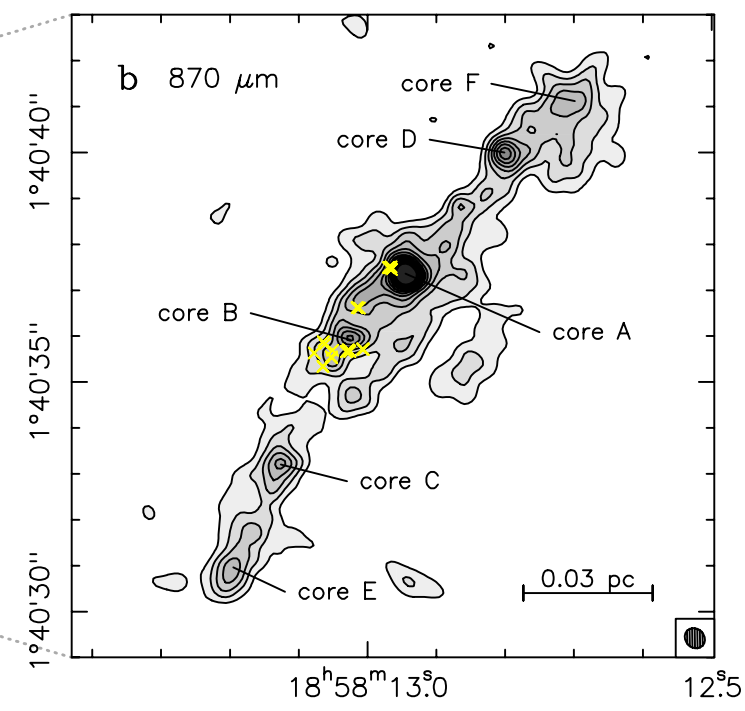

Fig. 1. a) Spitzer $4.5 \mu \mathrm{m}$ image (in logarithmic scale) of the star forming region G35.20-0.74 N, overlaid with a contour map of the $870 \mu \mathrm{m}$ (350 GHz) continuum emission obtained with ALMA. The IR image has been obtained by applying HiRes deconvolution (Velusamy et al. 2008) to the Spitzer/IRAC data. The submillimeter map has been corrected for primary beam attenuation. Contours start at $5 \sigma$, increasing in steps of $6 \sigma$ (with $\sigma=1.8 \mathrm{mJy}_{\text {beam }}^{-1}$ ), up to a maximum level of $201.7 \mathrm{mJy} \mathrm{beam}^{-1}$. The dashed white circle denotes the primary beam of the ALMA $12 \mathrm{~m}$ antennas $\left(\sim 9^{\prime \prime}\right)$, while the ALMA synthesized beam $\left(0 !^{\prime} 474 \times 00^{\prime} 411, \mathrm{PA}=46^{\circ}\right)$ is shown in the bottom-right corner of the panel. b) Close-up of the central region that shows the $870 \mu \mathrm{m}$ continuum emission map obtained with ALMA. Six cores (from A to F) have been identified and marked in the image (see Table 2). Yellow crosses mark the position of OH masers (Hutawarakorn \& Cohen 1999). The ALMA synthesized beam is shown in the bottom-right corner of the panel. The spatial scale is also indicated in both panels.

and imaged using $\mathrm{CASA}^{1}$ (McMullin et al. 2007). A continuum map was obtained from line-free channels and subtracted from the data, directly in the $(u, v)$-domain. Channel maps were created for some selected molecular transitions (see Table 1 and Sect. 3). Continuum and channel maps were created with the robust parameter of Brigg (1995) set equal to 0.5, as a compromise between resolution and sensitivity to extended sources. The resulting continuum image has a synthesized CLEANed beam of $0.47 \times 0.0^{\prime} 41, \mathrm{PA}=46^{\circ}$ and a rms noise of $1.8 \mathrm{mJy} \mathrm{beam}^{-1}$. The synthesized CLEANed beams of the spectral line maps vary from $0.50 \times 0 .{ }^{\prime} 42$ to $0 . .48 \times 0 .{ }^{\prime} 41$ with increasing frequency, with $\mathrm{PA}=40-45^{\circ}$. The rms noise of each spectral channel (of $0.6 \mathrm{~km} \mathrm{~s}^{-1}$ ) varies between 5 and $20 \mathrm{mJy} \mathrm{beam}^{-1}$, with the largest values measured in channels with strong line emission. The spectral line images were analyzed using the CLASS and GREG programs of the GILDAS ${ }^{2}$ software package developed by the IRAM and Observatoire de Grenoble. All the resulting spectra have been smoothed to a resolution of $0.6 \mathrm{~km} \mathrm{~s}^{-1}$.

\section{Results}

\subsection{Continuum emission}

In Fig. 1a, we show the map of the ALMA $870 \mu \mathrm{m}(350 \mathrm{GHz})$ continuum emission towards G35.20N overlaid on the Spitzer $4.5 \mu \mathrm{m}$ image, whose resolution has been enhanced with HiRes (Velusamy et al. 2008). The submillimeter continuum emission appears to trace an elongated structure in the SE-NW direction, perpendicular to the $4.5 \mu \mathrm{m}$ extended emission, and located

\footnotetext{
1 The Common Astronomy Software Applications (CASA) software can be downloaded at http: //casa.nrao.edu

2 http://wWw.iram.fr/IRAMFR/GILDAS
}

across the waist of the butterfly-shaped nebula seen in the infrared. With an extension of $\sim 0.15 \mathrm{pc}$ and a mean width of $\sim 0.013 \mathrm{pc}$ (full width at half maximum), this is probably the densest part of the elongated structure observed at larger scales (0.21 pc long and $0.13 \mathrm{pc}$ wide; Little et al. 1985; Brebner et al. 1987; Gibb et al. 2003). This structure is not smooth but fragmented in several smaller condensations. The 2D CLUMPFIND algorithm (Williams et al. 1994) identifies six of these, which we name cores A-F (see Fig. 1b), although additional substructure is seen in the map. The strongest of these, cores A and B, are located at the center of the elongated structure, and were already identified by Sánchez-Monge et al. (2013b). These two cores are associated with faint centimeter continuum emission likely tracing young H II regions or radiojets (see Gibb et al. 2003; Sánchez-Monge et al. 2013b), with a number of 1665MHz OH maser features (Brebner et al. 1987; Hutawarakorn \& Cohen 1999), and with probably ${ }^{3} 6.7 \mathrm{GHz} \mathrm{CH}_{3} \mathrm{OH}$ maser emission. Core $\mathrm{C}$, located $\sim 3^{\prime \prime}$ to the $\mathrm{SE}$ of core $\mathrm{B}$, coincides with the millimeter source G35MM2 reported by Gibb et al. (2003). The ALMA $870 \mu \mathrm{m}$ dust continuum emission shown in Fig. 1a is consistent with the Submillimeter Array (SMA) $880 \mu \mathrm{m}$ image obtained by Qiu et al. (2013, see their Fig. 1c). There are some morphological differences to the southeast of the elongated

3 The $6.7 \mathrm{GHz} \mathrm{CH}_{3} \mathrm{OH}$ maser observations carried out with the Japanese VLBI Network (JVN; Sugiyama et al. 2008) and the European VLBI Neatwork (EVN; Surcis et al. 2012) were performed without phase-referencing and it is thus impossible to establish the absolute position of the masers. However, the $\mathrm{CH}_{3} \mathrm{OH}$ maser distribution seems to match the OH maser distribution (Brebner et al. 1987; Hutawarakorn \& Cohen 1999); since the latter are associated with cores A and B, it seems likely that the methanol masers are also associated with these two cores. 
Table 1. Molecular transitions studied in this paper.

\begin{tabular}{|c|c|c|}
\hline Observation & $\begin{array}{c}\text { Frequency } \\
(\mathrm{MHz})\end{array}$ & $\begin{array}{c}E_{\text {upper }} / k^{a} \\
(\mathrm{~K})\end{array}$ \\
\hline $\mathrm{CH}_{3} \mathrm{OCHO}\left(27_{7,21}-26_{7,20}\right) \mathrm{A}$ & 335015.90 & 443.5 \\
\hline $\mathrm{CH}_{3} \mathrm{OH}\left(2_{2,1}-3_{1,2}\right)$ & 335133.57 & 44.7 \\
\hline${ }^{13} \mathrm{CH}_{3} \mathrm{OH}\left(12_{1,11}-12_{0,12}\right)$ & 335560.21 & 192.7 \\
\hline $\mathrm{CH}_{3} \mathrm{OH}\left(7_{1,7}-6_{1,6}\right)$ & 335582.02 & 79.0 \\
\hline $\mathrm{CH}_{3} \mathrm{OCHO}\left(27_{9,18}-26_{9,17}\right) \mathrm{A}$ & 336111.32 & 277.9 \\
\hline $\mathrm{CH}_{3} \mathrm{OH}\left(14_{7,8}-15_{6,9}\right)$ & 336438.21 & 488.2 \\
\hline $\mathrm{HC}_{3} \mathrm{~N}(37-36)$ & 336520.08 & 306.9 \\
\hline $\mathrm{CH}_{3} \mathrm{OH}\left(12_{1,11}-12_{0,12}\right)$ & 336865.15 & 197.1 \\
\hline $\mathrm{CH}_{3} \mathrm{OCHO}\left(26_{6,20}-25_{6,19}\right) \mathrm{E}$ & 336889.20 & 235.5 \\
\hline $\mathrm{CH}_{3} \mathrm{OCHO}\left(26_{6,20}-25_{6,19}\right) \mathrm{A}$ & 336918.18 & 235.5 \\
\hline $\mathrm{C}^{17} \mathrm{O}(3-2)$ & 337061.05 & 32.4 \\
\hline$C^{34} S(7-6)$ & 337396.69 & 50.2 \\
\hline $\mathrm{CH}_{3} \mathrm{OH}\left(7_{0,7}-6_{0,6}\right) v_{\mathrm{t}}=1$ & 337748.83 & 488.5 \\
\hline $\mathrm{CH}_{3} \mathrm{OH}\left(7_{1,6}-6_{1,5}\right) v_{\mathrm{t}}=1$ & 337969.44 & 390.1 \\
\hline $\mathrm{H}_{2} \mathrm{CS}\left(10_{1,10}-9_{1,9}\right)$ & 338083.20 & 102.4 \\
\hline $\mathrm{SO}_{2}\left(18_{4,14}-18_{3,15}\right)$ & 338305.99 & 196.8 \\
\hline $\mathrm{CH}_{3} \mathrm{OH}\left(7_{3,4}-6_{3,3}\right)$ & 338583.22 & 112.7 \\
\hline $\mathrm{CH}_{3} \mathrm{OH}\left(7_{2,5}-6_{2,4}\right)$ & 338639.80 & 102.7 \\
\hline $\mathrm{H}^{13} \mathrm{CO}^{+}(4-3)$ & 346998.34 & 41.6 \\
\hline${ }^{13} \mathrm{CH}_{3} \mathrm{OH}\left(14_{1,13}-14_{0,14}\right)$ & 347188.28 & 254.3 \\
\hline $\mathrm{SiO}(8-7)$ & 347330.63 & 75.0 \\
\hline $\mathrm{CH}_{3} \mathrm{OCHO}\left(28_{10,19}-27_{10,18}\right) \mathrm{E}$ & 347628.34 & 306.8 \\
\hline $\mathrm{CH}_{3} \mathrm{OCHO}\left(28_{6,23}-27_{6,2}\right) \mathrm{E}$ & 348049.89 & 266.1 \\
\hline $\mathrm{CH}_{3} \mathrm{OCHO}\left(28_{6,23}-27_{6,2}\right) \mathrm{A}$ & 348065.97 & 266.1 \\
\hline $\mathrm{H}_{2} \mathrm{CS}\left(10_{1,9}-9_{1,8}\right)$ & 348534.36 & 105.2 \\
\hline $\mathrm{CH}_{3} \mathrm{OH}\left(14_{1,13}-14_{0,14}\right)$ & 349107.00 & 260.2 \\
\hline $\mathrm{CH}_{3}{ }^{13} \mathrm{CN}\left(19_{K}-18_{K}\right)$ & $349280.77^{b}$ & $\ldots$ \\
\hline $\mathrm{CH}_{3} \mathrm{CN}\left(19_{K}-18_{K}\right)$ & $349453.70^{b}$ & \\
\hline $\operatorname{HNCO}\left(16_{1,16}-15_{1,15}\right)$ & 350333.35 & 186.2 \\
\hline $\mathrm{CH}_{3} \mathrm{CN}(K, l) v_{8}=1$ & $350552.44^{c}$ & $\ldots$ \\
\hline
\end{tabular}

Notes. ${ }^{(a)}$ The frequencies are obtained from the Cologne Database for Molecular Spectroscopy (CDMS; Müller et al. 2001) and Jet Propulsion Laboratory (JPL; Pickett et al. 1998) catalogs. $E_{\text {upper }}$ is the upper level energy, and $k$ the Boltzmann constant. ${ }^{(b)}$ The frequency is that of the $K=0$ component. ${ }^{(c)}$ The frequency is that of the $K, l=2,+1$ component.

structure, probably due to the vicinity of the primary beam edge in our ALMA observations.

In Table 2, we list for all the cores the coordinates of the peak position, the primary beam corrected fluxes, and the deconvolved sizes. The fluxes have been computed by integrating the emission within the $5 \sigma$ contour level. The peak intensities range from $40 \mathrm{mJy}^{\text {beam }}{ }^{-1}$ to $200 \mathrm{mJy}^{\text {beam }}{ }^{-1}$, while the integrated fluxes are in the range $80-700 \mathrm{mJy}$. These fluxes are slightly lower (a factor of 2) than those measured by Qiu et al. (2013) in their SMA observations. However, we note that Qiu et al. determine the flux densities for larger dust entities containing at least two cores (e.g., their object MM1, with a total flux of $3 \mathrm{Jy}$, is the sum of cores A and B). We have convolved the ALMA image to the beam of the SCUBA $850 \mu \mathrm{m}$ image (15"; Qiu et al. 2013), and compared the peak fluxes of both images $\left(\sim 2.5 \mathrm{Jy}\right.$ beam $^{-1}$ versus $\left.\sim 8.5 \mathrm{Jy} \mathrm{beam}^{-1}\right)$ to conclude that we are recovering around $30 \%$ of the flux measured with SCUBA. The deconvolved diameters of the dust cores (see Table 2) have been computed from the $50 \%$ contour level applying Gaussian deconvolution (see procedure described in Table 3 of Sánchez-Monge et al. 2013a). The mean deconvolved diameter is $\sim 1600 \mathrm{AU}$, with core A being the most compact (900 AU), and core $\mathrm{F}$ the most extended (2600 AU). The different cores appear regularly spaced, with a mean (projected) separation of $\sim 2$ '. 1 or $\sim 0.023$ pc.

\subsection{Line emission}

In Fig. 2, we show the spectra obtained by averaging the line emission over the cores in G35.20N (lower sideband: 334 965.73-338723.83 MHz, and upper sideband: 346891.29 $350718.05 \mathrm{MHz})$. Cores $\mathrm{A}$ and $\mathrm{B}$ show rich spectra, with emission of several complex organic molecules (e.g., $\mathrm{CH}_{3} \mathrm{CN}$, $\mathrm{CH}_{3} \mathrm{OH}, \mathrm{CH}_{3} \mathrm{OCHO}, \mathrm{NH}_{2} \mathrm{CHO}, \mathrm{HC}_{3} \mathrm{~N}, \mathrm{C}_{2} \mathrm{H}_{5} \mathrm{OH}, \mathrm{CH}_{3} \mathrm{OCHO}$ ). Out of the rest of the cores, only core $\mathrm{C}$ is likely associated with emission in complex molecules such as $\mathrm{CH}_{3} \mathrm{CN}$ and $\mathrm{CH}_{3} \mathrm{OH}$. Core $\mathrm{C}$ was found to be associated with molecular emission at millimeter wavelengths in previous studies (e.g., Gibb et al. 2003; Birks et al. 2006). The rest of the cores are only associated with faint emission of more abundant species such as $\mathrm{C}^{17} \mathrm{O}$, $\mathrm{C}^{34} \mathrm{~S}, \mathrm{H}^{13} \mathrm{CO}^{+}$(see Fig. 3) and weak $\mathrm{CH}_{3} \mathrm{OH}$, suggesting that these cores could either host only low-mass star formation, or be in an evolutionary phase prior to the hot core stage.

\subsubsection{Large-scale molecular emission}

Out of all the species detected in the region (mainly associated with cores $\mathrm{A}$ and $\mathrm{B}$; see Sect. 3.2.2), there are four molecules $\left(\mathrm{C}^{17} \mathrm{O}, \mathrm{C}^{34} \mathrm{~S}, \mathrm{H}^{13} \mathrm{CO}^{+}\right.$and $\mathrm{SiO}$ ) that show a complex (extended) spatial distribution. Their emission appears partially resolved out by the interferometer, which is sensitive to angular scales $\approx 2^{\prime \prime}$ (see Sect. 2). In order to improve the detection of the extended emission, we generated new CLEANed maps for these four species after tapering the data at $1^{\prime \prime}$ resolution. In Fig. 3, we show the corresponding integrated emission (zeroth-order moment) maps, over the velocity range 23 to $42 \mathrm{~km} \mathrm{~s}^{-1}$, and compare them with the dust continuum emission. For these molecules, the line emission coincides with the dust continuum emission, with $\mathrm{C}^{17} \mathrm{O}$ and $\mathrm{H}^{13} \mathrm{CO}^{+}$being detected toward all the cores and tracing the elongated structure, and $\mathrm{SiO}$ and $\mathrm{C}^{34} \mathrm{~S}$ mostly concentrated towards cores A and B, consistent with the SMA images reported by Qiu et al. (2013). We estimate a missing flux of $50 \%$ from the comparison of our ALMA $\mathrm{H}^{13} \mathrm{CO}^{+}$ image and the map obtained by Qiu et al. using compact configurations of the SMA. Such an elongated structure has also been revealed at larger scales $\left(\sim 40^{\prime \prime}\right)$ in the $\mathrm{H}^{13} \mathrm{CO}^{+}(1-0)$ and $\mathrm{H}^{13} \mathrm{CN}$ (1-0) lines (e.g., Gibb et al. 2003). In Sect. 4.5, we will analyze the kinematics of this extended molecular structure.

\subsubsection{Compact molecular emission: hot cores}

As shown in Fig. 2, cores A and B show a very rich chemistry with strong emission of many species typical of hot cores. Core $\mathrm{C}$ also shows emission in complex molecules, although with an intensity several times fainter than the two cores located at the center of the elongated structure. A detailed analysis of the molecular content towards these three cores will be the subject of a forthcoming paper. In the present study, we will only focus on a few hot core tracers, basically those that are clearly detected, and less blended with other species: $\mathrm{CH}_{3} \mathrm{CN}, \mathrm{CH}_{3}{ }^{13} \mathrm{CN}$, and vibrationally excited $\mathrm{CH}_{3} \mathrm{CN}$, as well as $\mathrm{CH}_{3} \mathrm{OH}, \mathrm{CH}_{3} \mathrm{OCHO}$, $\mathrm{HC}_{3} \mathrm{~N}, \mathrm{HNCO}, \mathrm{H}_{2} \mathrm{CS}$, and $\mathrm{SO}_{2}$. Similar to the large-scale molecular and continuum emission, we have estimated the missing flux in our maps of hot-core tracers by comparing the ALMA $\mathrm{CH}_{3} \mathrm{CN} \mathrm{K}=0,1$ lines with the same lines observed with the SMA in compact configurations (Qiu et al. 2013) and with the 
Table 2. Parameters of the cores detected in the $870 \mu \mathrm{m}$ ALMA continuum map (see Fig. 1).

\begin{tabular}{|c|c|c|c|c|c|c|}
\hline \multirow[b]{2}{*}{ Core } & \multicolumn{2}{|c|}{ Peak position } & \multirow[b]{2}{*}{$\begin{array}{c}I_{\text {peak }}{ }^{a} \\
\left(\text { Jy beam }^{-1}\right)\end{array}$} & \multirow[b]{2}{*}{$\begin{array}{l}S_{v}{ }^{a} \\
(\mathrm{Jy})\end{array}$} & \multicolumn{2}{|c|}{ Source diameter ${ }^{b}$} \\
\hline & $\begin{array}{c}\alpha(J 2000) \\
\mathrm{h} \mathrm{m} \mathrm{s}\end{array}$ & $\begin{array}{c}\delta(J 2000) \\
\circ, 1,\end{array}$ & & & $\begin{array}{c}\theta_{\mathrm{S}} \\
(\operatorname{arcsec})\end{array}$ & $\begin{array}{c}D_{\mathrm{S}} \\
(\mathrm{AU})\end{array}$ \\
\hline $\mathrm{A}$ & 185812.945 & 014037.36 & 0.208 & $0.692 \pm 0.023$ & 0.414 & 900 \\
\hline B & 185813.025 & 014035 . & 0.0 & $0.333 \pm 0.0$ & 0.838 & 1800 \\
\hline $\mathrm{C}$ & 185813.126 & 014033.20 & 0.056 & $0.190 \pm 0.006$ & 0.717 & 1600 \\
\hline $\mathrm{D}$ & 185812.801 & 014040.00 & 0.083 & $0.229 \pm 0.007$ & 0.571 & 1300 \\
\hline $\mathrm{E}$ & 185813.195 & 014030.96 & 0.048 & $0.162 \pm 0.005$ & 0.842 & 1900 \\
\hline $\mathrm{F}$ & 185812.699 & 014041.12 & 0.049 & $0.276 \pm 0.006$ & 1.181 & 2600 \\
\hline
\end{tabular}

Notes. ${ }^{(a)}$ Primary beam corrected peak intensity $\left(I_{\text {peak }}\right)$ and flux density $\left(S_{v}\right)$ integrated within the $5 \sigma$ contour level. ${ }^{(b)}$ Deconvolved average diameter of the 50\% contour level (following Sánchez-Monge et al. 2013a).

Atacama Submillimeter Telescope Experiment (ASTE; Furuya, priv. comm.). The ALMA observations recover around $75 \%$ of the flux measured with ASTE. The fluxes between the SMA and ALMA images (after convolution to the same beam) are equal within a $8 \%$.

Figures B.1-B.3 show a close-up view of the $\mathrm{CH}_{3} \mathrm{CN}$ and $\mathrm{CH}_{3} \mathrm{OH}$ transitions detected towards cores $\mathrm{A}, \mathrm{B}$ and $\mathrm{C}$, respectively. The observational setup (see Sect. 2) permits to cover up to the $K=9$ transition of $\mathrm{CH}_{3} \mathrm{CN}\left(19_{K}-18_{K}\right)$, and up to the $K=5$ line of $\mathrm{CH}_{3}{ }^{13} \mathrm{CN}\left(19_{K}-18_{K}\right)$, with upper level energies of $745 \mathrm{~K}$ and $517 \mathrm{~K}$, respectively. All of these $\mathrm{CH}_{3} \mathrm{CN}$ lines have been detected in the three cores. Regarding the $\mathrm{CH}_{3}{ }^{13} \mathrm{CN}$, only a few lines are not blended (e.g., $K=2, K=4$ ), while others clearly overlap with methyl cyanide or methanol lines (e.g., $K=3, K=5$ ). Several vibrationally excited $\mathrm{CH}_{3} \mathrm{CN}$ transitions, with upper level energies ranging from $\sim 600 \mathrm{~K}$ to $\sim 1100 \mathrm{~K}$, have been detected. The intensity of the methyl cyanide lines in core $\mathrm{A}$ is almost twice the intensity of the lines in core B, and ten times stronger than in core $\mathrm{C}$. A large number of methanol $\left(\mathrm{CH}_{3} \mathrm{OH}\right)$ and methyl formate $\left(\mathrm{CH}_{3} \mathrm{OCHO}\right)$ lines have been detected in these three cores, but in the following, we have only considered those transitions that are not blended with other (strong) lines. The selected lines are listed in Table 1, and cover a range of upper level energies from $45 \mathrm{~K}$ to $488 \mathrm{~K}$ in the case of methanol, and from $236 \mathrm{~K}$ to $444 \mathrm{~K}$ for methyl formate. Finally, we have also selected some strong and isolated lines of different species such as $\mathrm{H}_{2} \mathrm{CS}, \mathrm{HC}_{3} \mathrm{~N}, \mathrm{SO}_{2}$ or $\mathrm{HNCO}$.

In Figs. 4, A.1 and A.2, we present the zeroth (integrated intensity) and first (velocity field) moment maps of different hot core species detected towards cores $\mathrm{A}$ and $\mathrm{B}: \mathrm{CH}_{3} \mathrm{CN} K=2$ and $8, \mathrm{CH}_{3}{ }^{13} \mathrm{CN} K=2$, and $\mathrm{CH}_{3} \mathrm{CN} v_{8}=1 K, l=3,1$, as well as different transitions of $\mathrm{CH}_{3} \mathrm{OH}, \mathrm{CH}_{3} \mathrm{OCHO}, \mathrm{H}_{2} \mathrm{CS}, \mathrm{HNCO}$, $\mathrm{SO}_{2}$, and $\mathrm{HC}_{3} \mathrm{~N}$. The moment maps have been computed following the same procedure as in Sánchez-Monge et al. (2013b), using the velocity interval $25.9-38.0 \mathrm{~km} \mathrm{~s}^{-1}$ for core A, and 25.9-33.0 $\mathrm{km} \mathrm{s}^{-1}$ for core B. As seen in the maps, the line emission peak in core A is perfectly coincident with the position of the dust emission peak. In both the molecular and continuum images, the emission of core $\mathrm{A}$ is barely resolved with a mean deconvolved diameter of $\sim 1100$ AU ( 0.'52), obtained from a 2D Gaussian fit to the zero-order moment maps, and applying Gaussian deconvolution. In core $\mathrm{B}$, the strongest peak of the molecular emission is typically located to the SE of the dust peak. For most of the transitions, the molecular emission of core B is clearly resolved (deconvolved size $\sim 0.95 ; \sim 2000$ AU) in an elongated structure oriented in the SE-NW direction, with the dust peak located close to the center of the extended line emission. For both cores, the emission is more compact in the maps of high excitation energy transitions (e.g., $\mathrm{CH}_{3} \mathrm{CN} v_{8}=1$, or $\mathrm{CH}_{3} \mathrm{OCHO}$ at $335.015 \mathrm{GHz}$ ) or less abundant species (e.g., $\mathrm{CH}_{3}{ }^{13} \mathrm{CN} K=2$ ), suggesting the existence of temperature and/or molecular abundance gradients within the cores. This is better seen in Fig. 6, where we plot the deconvolved size for different transitions against the upper level energy of the transition. For both cores, the trend is such that the higher the excitation energy of the transition is, the more compact the emission is. From the velocity field maps, we see a clear velocity gradient in both cores for all the species. The velocity gradient of core B is oriented in the $(\mathrm{S}) \mathrm{SE}-(\mathrm{N}) \mathrm{NW}$ direction, while the velocity gradient in core $\mathrm{A}$ is closer to the $\mathrm{N}-\mathrm{S}$ direction. We also note that the velocity gradient in both cores is reversed, with redshifted emission to the north for core B and to the south for core A. A more detailed analysis of the kinematics of these two cores is presented in Sect. 4.3.

Finally, in Fig. 5, we present the integrated intensity and velocity field maps of four transitions of hot-core species towards core $\mathrm{C}$ : $\mathrm{CH}_{3} \mathrm{CN}\left(19_{2}-18_{2}\right), \mathrm{CH}_{3} \mathrm{OH}\left(2_{2,1}-3_{1,2}\right), \mathrm{H}_{2} \mathrm{CS}\left(10_{1,9}-\right.$ $9_{1,8}$ ), and $\mathrm{HC}_{3} \mathrm{~N}(37-36)$. The moments were computed using the velocity interval $26.1-41.0 \mathrm{~km} \mathrm{~s}^{-1}$. Similar to core A, the molecular emission in core $\mathrm{C}$ is compact and coincident with the dust emission. Differently from cores A and B, we do not find a clear velocity gradient in any of the molecules.

\subsubsection{High-velocity SiO outflow emission}

We have analyzed the $\mathrm{SiO}(8-7)$ line emission to search for outflow signatures, since this molecule is known to be one of the best jet/outflow tracers in star forming regions (e.g., Gusdorf et al. 2008a,b; López-Sepulcre et al. 2011; Sánchez-Monge et al. 2013d). Channel maps of the $\mathrm{SiO}(8-7)$ emission are displayed in Fig. 7, with the $870 \mu \mathrm{m}$ continuum emission and hot core $\left(\mathrm{CH}_{3} \mathrm{CN}\right)$ line emission overlaid. The $\mathrm{SiO}$ emission appears to span a wide range of velocities, however, line blending in hot cores A and B hinders the identification of high-velocity emission at the position of the two cores.

To overcome this problem, we identified three different regions to search for high-velocity $\mathrm{SiO}$ emission. These regions (named "out-S", "out-SW" and "out-NE") are located far from the cores identified in Fig. 1, thus avoiding contamination from the chemically-rich hot cores (see Fig. 7). The spectra of the emission integrated over the three regions are shown in Fig. 8, together with the spectra of the $\mathrm{C}^{17} \mathrm{O}(3-2)$ emission as a reference of the dense gas emission. The $\mathrm{SiO}$ emission at the "out-NE" position, spanning a range from 32 up to $60 \mathrm{~km} \mathrm{~s}^{-1}$, is clearly redshifted with respect to the velocities of cores A and B (31.9 and $29.8 \mathrm{~km} \mathrm{~s}^{-1}$, respectively), and to the $\mathrm{C}^{17} \mathrm{O}$ line emission (with 

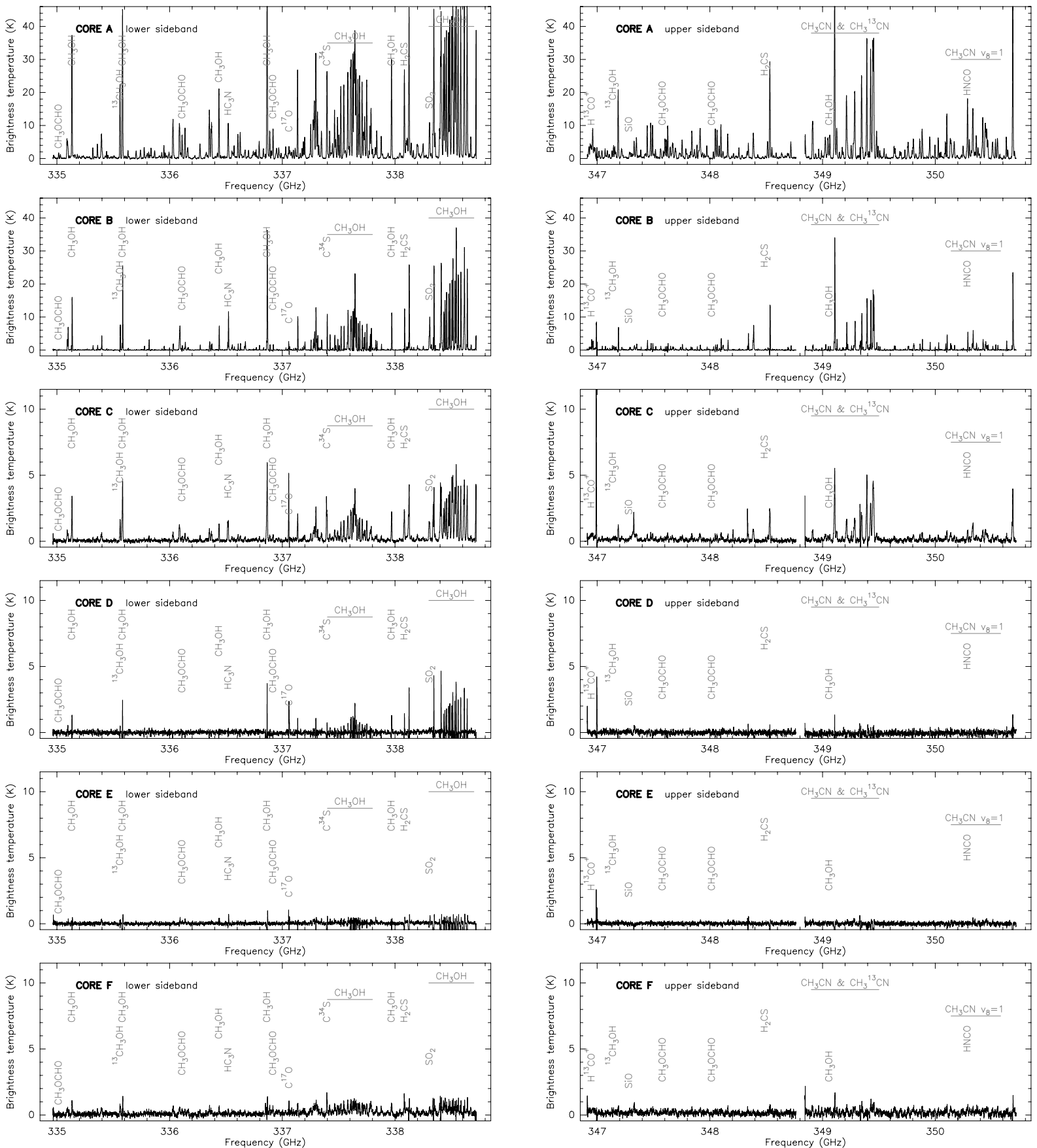

Fig. 2. Spectra of the full frequency range surveyed by the ALMA observations, toward the six cores identified in the continuum image (see Fig. 1). The spectra are extracted by averaging the intensity over an area of 1.37, 2.35, 1.04, 0.55, 0.75, $0.44 \operatorname{arcsec}^{2}$, yielding conversion factors of 8.9, $5.0,11.3,21.7,15.7,27.1 \mathrm{~K} \mathrm{Jy}^{-1}$, for cores A to F respectively. The brightness temperature scale of cores A and B is different from that of the rest of the cores. The absorption seen in some spectra is likely produced by the filtering of extended emission.

the peak at $\sim 32.3 \mathrm{~km} \mathrm{~s}^{-1}$ ). In the "out-S" position, the $\mathrm{SiO}$ emission spans a range of velocities from 0 up to $32 \mathrm{~km} \mathrm{~s}^{-1}$, i.e., blue-shifted with respect to the $\mathrm{C}^{17} \mathrm{O}$ line emission. Finally, at the "out-SW" position, the $\mathrm{SiO}$ line has a narrow profile similar to the $\mathrm{C}^{17} \mathrm{O}$ line, both in velocity and linewidth. This suggests that the $\mathrm{SiO}$ emission at this position is associated with to the large-scale dense gas emission, rather than with high-velocity outflows.

In Fig. 9, we show the zeroth-order moment maps of the $\mathrm{SiO}$ emission integrated over two different velocity ranges: (1) blue-shifted velocities in the range $25.2-28.6 \mathrm{~km} \mathrm{~s}^{-1}$; and
(2) red-shifted velocities in the range $40.8-57.7 \mathrm{~km} \mathrm{~s}^{-1}$. These velocity ranges have been chosen to avoid contamination of the hot core species associated with cores A and B (see middle panel in Fig. 8). The blue-shifted emission is located in between cores $\mathrm{A}$ and $\mathrm{B}$, and thus it is difficult to determine the powering source. On the other hand, $\mathrm{SiO}$ red-shifted emission seems to be clearly emanating from core A. While the configuration of the $\mathrm{SiO}$ high-velocity emission does not resemble a bipolar outflow, it is worth comparing the $\mathrm{SiO}$ map with that of the free-free $3.6 \mathrm{~cm}$ continuum obtained by Gibb et al. (2003) with the Very Large Array (see black contours in Fig. 9). One sees two radio 

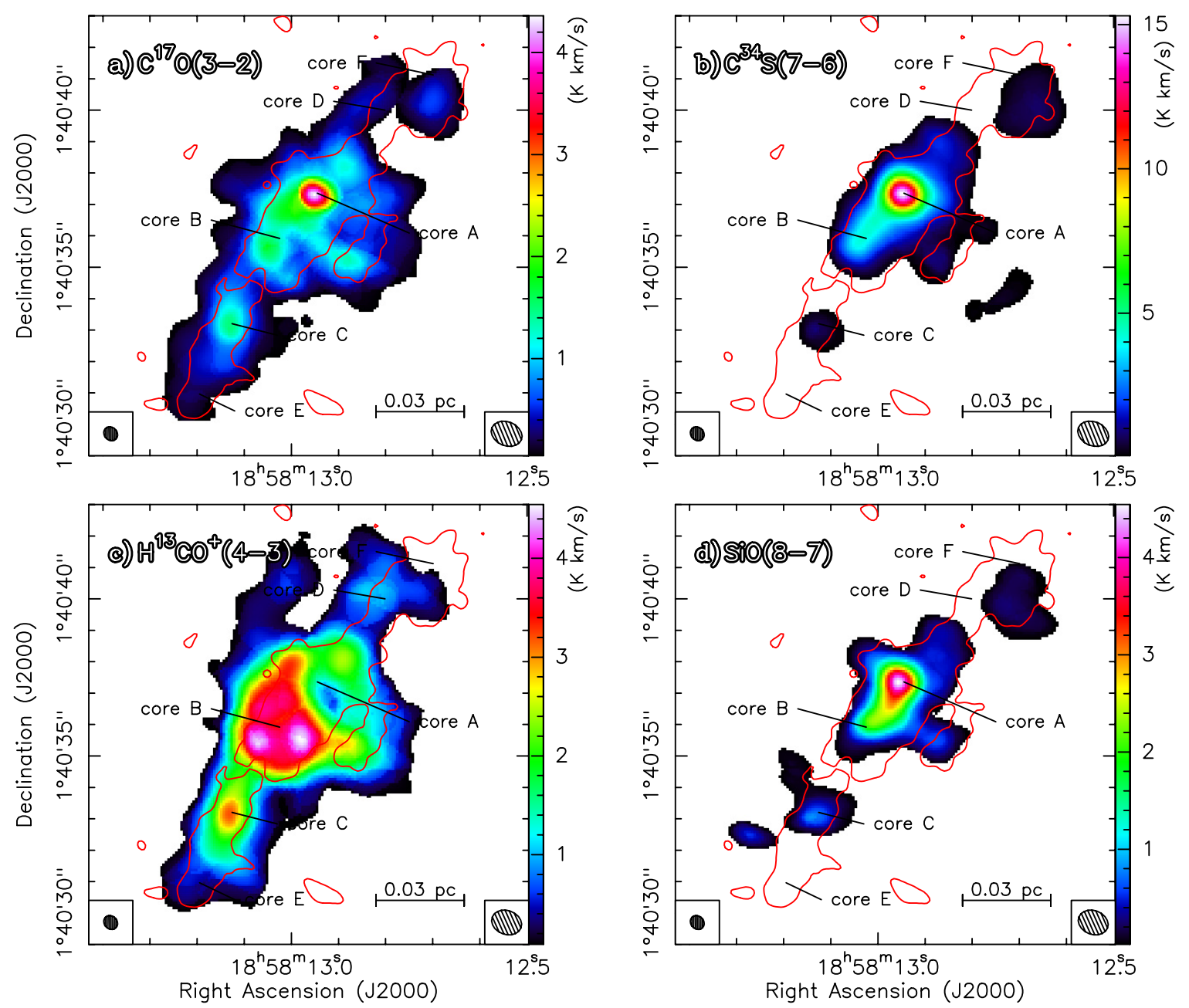

Fig. 3. Overlay of the $870 \mu \mathrm{m}$ continuum emission (red contour) on the integrated intensity maps (colors) of $\mathrm{C}^{17} \mathrm{O}, \mathrm{C}^{34} \mathrm{~S}, \mathrm{H}^{13} \mathrm{CO}^{+}$, and $\mathrm{SiO}$. The contour level of the continuum emission corresponds to the $9.0 \mathrm{mJy}^{\mathrm{beam}}{ }^{-1}$ intensity (see Fig. 1). The intensity scale for the integrated emission for each molecule is shown in the color bar at the right of each panel, in $\mathrm{K} \mathrm{km} \mathrm{s}^{-1}$. The synthesized beam of the continuum emission $\left(0 . ' 474 \times 0 \prime^{\prime} 411, \mathrm{PA}=46^{\circ}\right)$ is shown in the bottom-left corner of each panel. For each molecule, we show the synthesized beam of the tapered

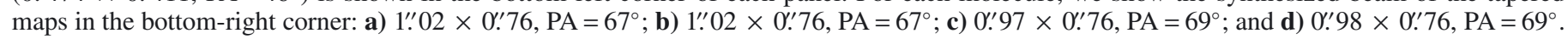

peaks that are lined with core $\mathrm{A}$ and the red-shifted $\mathrm{SiO}$ emission, suggesting that both the radio continuum and $\mathrm{SiO}$ emission might be tracing a jet from the core, oriented in the SW-NE direction.

In the following we test if the $\mathrm{SiO}$ and centimeter continuum emission could be arising from the same outflow. Anglada (1996) found an observational relation between the outflow momentum rate, $\dot{P}$, and the radio continuum luminosity, $S_{v} d^{2}$, of the jet associated with the outflow. This observational relation is in agreement with the shock induced ionization mechanism proposed by Curiel et al. (1989) to explain the radio emission of the jets. The relation reads as

$\left[\frac{S_{v} d^{2}}{\mathrm{mJy} \mathrm{kpc}^{2}}\right]=10^{3.5}\left[\frac{\Omega}{4 \pi}\right]\left[\frac{\dot{P}}{M_{\odot} \mathrm{yr}^{-1} \mathrm{~km} \mathrm{~s}^{-1}}\right]$,

where $\Omega / 4 \pi$ is an efficiency factor that indicates the fraction of the solid angle that is shocked $(\sim 0.1$ for low-mass radiojets/outflows, Anglada 1996), $S_{v}$ is the flux density at $5 \mathrm{GHz}$, and $d$ the heliocentric distance. The flux density of the $3.6 \mathrm{~cm}$ continuum emission of the radiojet is $\sim 0.25-0.40 \mathrm{mJy}$ (depending on the sources/knots considered), which corresponds to a flux density of $\sim 0.18-0.30 \mathrm{mJy}$ at $5 \mathrm{GHz}$, assuming a typical spectral index of 0.6 . This results in an outflow momentum rate of
2.7-4.5 $\times 10^{-3} M_{\odot} \mathrm{yr}^{-1} \mathrm{~km} \mathrm{~s}^{-1}$. We compared this number with the outflow momentum rate that can be computed from the $\mathrm{SiO}$ high-velocity emission. We followed the same procedure described in López-Sepulcre et al. (2009), and derived an outflow momentum rate of $1.5 \times 10^{-3}$ and $0.4 \times 10^{-3} M_{\odot} \mathrm{yr}^{-1} \mathrm{~km} \mathrm{~s}^{-1}$, for the red and blue-shifted emission lobes shown in Fig. 9, assuming a $\mathrm{SiO}$ abundance of $3 \times 10^{-9}$ (Sánchez-Monge et al. 2013d). The derived outflow momentum rate for the red-shifted lobe agrees, within a factor of $2-3$, with that obtained from the radio continuum emission. The small discrepancy may be related to the uncertainties involved in these calculations (e.g., $\mathrm{SiO}$ abundance, $\Omega / 4 \pi$ efficiency factor). Observations, sensitive to scales $\sim 2$, , of different outflow tracers should be performed to confirm the scenario in which core $\mathrm{A}$ is powering an outflow-jet directed approximately NE-SW.

\subsection{Spectral energy distribution}

Figure 10 shows the spectral energy distribution (SED) of G35.20N, obtained from the Herschel infrared Galactic Plane Survey (Hi-GAL; Molinari et al. 2010a,b) images complemented with literature and archival data at wavelengths ranging from mid-infrared to millimeter. In particular, we used 

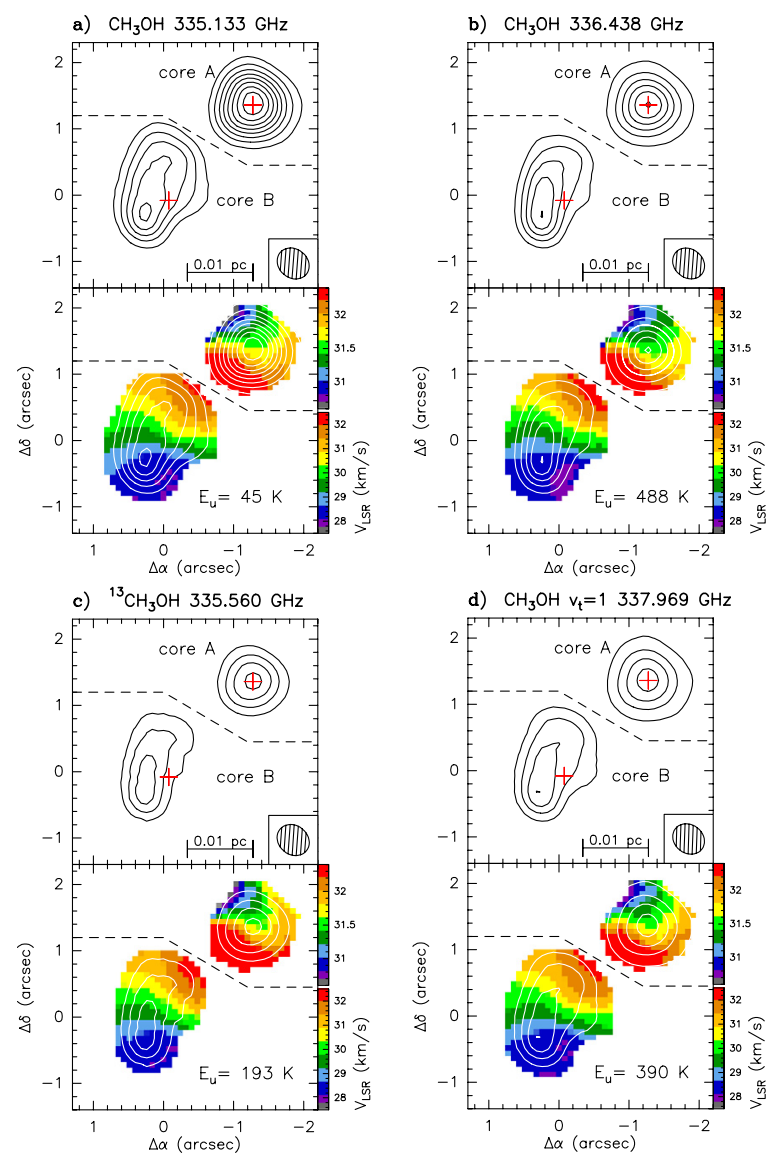

Fig. 4. Maps of the integrated intensity (contours) and velocity field (color maps in the bottom panels) of a) $\mathrm{CH}_{3} \mathrm{OH}\left(2_{2,1}-3_{1,2}\right)$, b) $\left.\mathrm{CH}_{3} \mathrm{OH}\left(14_{7,8}-15_{6,9}\right), \mathbf{c}\right){ }^{13} \mathrm{CH}_{3} \mathrm{OH}\left(12_{1,11}-12_{0,12}\right)$, and d) $\mathrm{CH}_{3} \mathrm{OH} v_{\mathrm{t}}=1$ $\left(7_{1,6}-6_{1,5}\right)$ towards cores $\mathrm{A}$ and $\mathrm{B}$. For the different panels, the starting (and increasing contour levels in terms of $\sigma$, in Jy beam ${ }^{-1} \mathrm{~km} \mathrm{~s}^{-1}$ ) are $5 \sigma(5 \sigma$, with $\sigma=0.18), 5 \sigma(10 \sigma$, with $\sigma=0.11), 5 \sigma$ ( $5 \sigma$, with $\sigma=0.25)$, and $5 \sigma(10 \sigma$, with $\sigma=0.19)$ for core A; and $5 \sigma(5 \sigma$, with $\sigma=0.11), 5 \sigma(5 \sigma$, with $\sigma=0.06), 3 \sigma$ (3o, with $\sigma=0.14)$, and $5 \sigma$ ( $5 \sigma$, with $\sigma=0.11$ ) for core B. Offsets are measured with respect to the phase center (see Sect. 2). The red crosses indicate the position of the dust emission peaks of cores A and B (see Table 2). The synthesized beam and spatial scale are shown in the bottom-right corner (upper panels). The energy of the upper level of each transition is indicated in the bottom-right corner (lower panels). The dashed line is intended to stress that the velocity intervals used to compute the moment maps are different for cores A and B.

the following data: Galactic Legacy Infrared Mid-Plane Survey Extraordinaire (GLIMPSE; Benjamin et al. 2003); Midcourse Space Experiment (MSX; Price et al. 1999); MIPSGAL/Spitzer (Carey et al. 2009); Wide-field Infrared Survey Explorer (WISE; Wright et al. 2010); APEX Telescope Large Area Survey of the Galaxy (ATLASGAL; Schuller et al. 2009); Mooney et al. (1995); De Buizer (2006); Zhang et al. (2013). We stress that inclusion of the Hi-GAL data at 70 and $160 \mu \mathrm{m}$, represents an important improvement with respect to previous estimates, as it allows us to sample the SED around the peak, i.e., the spectral region responsible for most of the bolometric luminosity.

The bolometric luminosity has been derived by integrating the SED over the full observed spectral distribution. We note that different spatial apertures have been used at each wavelength due to the different angular resolution of the data. We obtain $\sim 3 \times 10^{4} L_{\odot}$, thus confirming the value quoted in the rms
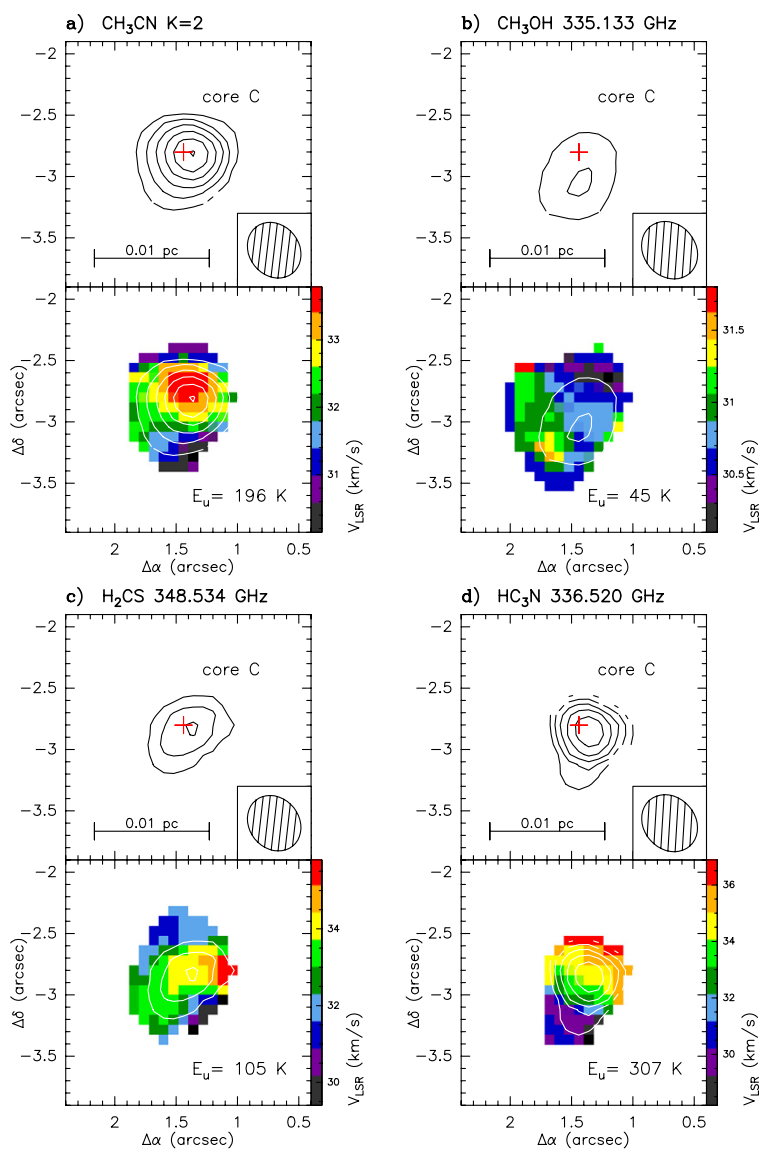

Fig. 5. Maps of the integrated intensity (upper panels) and velocity field (bottom panels) of a) $\mathrm{CH}_{3} \mathrm{CN} K=2$ at $349.426 \mathrm{GHz}$, b) $\mathrm{CH}_{3} \mathrm{OH}\left(2_{2,1}-\right.$ $\left.3_{1,2}\right)$, c) $\mathrm{H}_{2} \mathrm{CS}\left(10_{1,9}-9_{1,8}\right)$, and d) $\mathrm{HC}_{3} \mathrm{~N}(37-36)$ towards core C. The starting (and increasing) contour levels for the four panels are $1 \sigma(1 \sigma)$, with $\sigma$ equal to $0.18,0.22,0.20$ and $0.14 \mathrm{Jy} \mathrm{beam}^{-1} \mathrm{~km} \mathrm{~s}^{-1}$, for the four panels. Other symbols as in Fig. 4.

database ${ }^{4}$, but less than the luminosity $\left(\sim 0.7-2.2 \times 10^{5} L_{\odot}\right)$ derived by Zhang et al. (2013) from a model taking into account the photons which may escape through the outflow cavity (the so-called flashlight effect), but considering a single high-mass protostar forming within a massive core of radius $0.1 \mathrm{pc}$ (which seems to be inconsistent with the fragmentation seen in our highangular resolution observations).

\section{Analysis and discussion}

\subsection{Temperature and mass estimates}

The large number of methyl cyanide $\left(\mathrm{CH}_{3} \mathrm{CN}\right)$ transitions detected towards cores $\mathrm{A}, \mathrm{B}$ and $\mathrm{C}$, together with the methanol $\left(\mathrm{CH}_{3} \mathrm{OH}\right)$ transitions found in almost all the cores, can be used to obtain an estimate of the temperature of the cores in G35.20N. We fitted the observed methyl cyanide and methanol spectra for all the cores using the CASA interface of the XCLASS software called myXCLASS ${ }^{5}$ described in Schilke et al. (2001), Comito et al. (2005) and Zernickel et al. (2012). The myXCLASS software includes entries from the CDMS (Cologne Database

\footnotetext{
4 The rms database is available at http://www.ast. leeds.ac.uk/ cgi-bin/RMS/RMS_DATABASE.cgi

5 myXCLASS can be downloaded from https://www.astro. uni-koeln. de/projects/schilke/myXCLASSInterface
} 


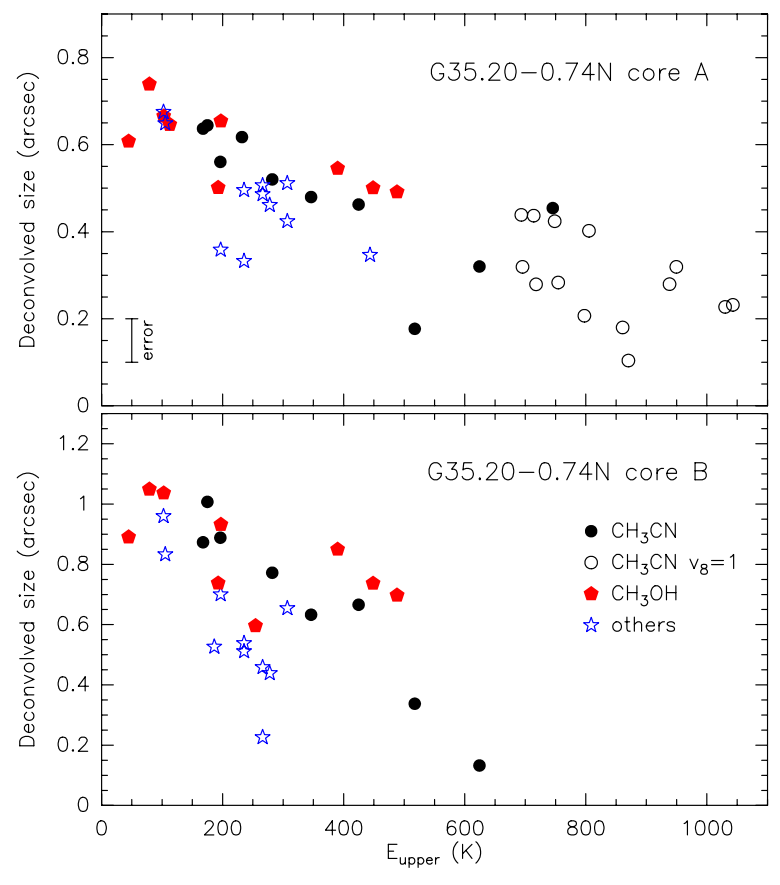

Fig. 6. Deconvolved size (in arcsec) for different molecular transitions (listed in Table 1) versus the upper level energy of the transition for cores A (top panel) and B (bottom panel). In both panels, black circles correspond to $\mathrm{CH}_{3} \mathrm{CN}$, open circles to $\mathrm{CH}_{3} \mathrm{CN}$ vibrationally excited, red pentagons to $\mathrm{CH}_{3} \mathrm{OH}$, and blue stars to transitions of other molecules. The typical error in size is shown in the top panel.

for Molecular Spectroscopy; Müller et al. 2001) and JPL (Jet Propulsion Laboratory; Pickett et al. 1998) catalogs. With this information, it is possible to simulate a spectrum of a molecule, by providing parameters such as the excitation temperature, abundance, size of the source, linewidth and velocity. Once an initial guess is set up, the parameters can be fitted using the program MAGIX (Modeling and Analysis Generic Interface for eXternal numerical codes; Bernst et al. 2011; Möller et al. 2013). Figures B.1 to B.4 show the observed and myXCLASS fitted $\mathrm{CH}_{3} \mathrm{CN}$ and $\mathrm{CH}_{3} \mathrm{OH}$ spectra towards the cores in $\mathrm{G} 35.20 \mathrm{~N}$. We fitted each molecule with a single component, i.e., the five free parameters are the same for all the lines of a given species. A more complex model, taking into account temperature and density gradients, might be necessary to explain the variations in size shown in Fig. 6. Finally, we note that the myXCLASS software takes the line opacity into account. The fits do not include only ground state transitions of the two species, but also vibrationally and torsionally excited as well as transitions from isotopologues such as $\mathrm{CH}_{3}^{13} \mathrm{CN}$ and ${ }^{13} \mathrm{CH}_{3} \mathrm{OH}$. We assumed the relative abundance $\left[{ }^{12} \mathrm{C} /{ }^{13} \mathrm{C}\right]$ to be 50 for a galactocentric distance of $6.5 \mathrm{kpc}$ (Wilson \& Rood 1994). The parameters of the best fits are listed in Table 3 . The temperature ranges typically between $100-300 \mathrm{~K}$ for cores A-D, in both methanol and methyl cyanide species. For cores $\mathrm{A}$ and $\mathrm{C}$, the estimated $\mathrm{CH}_{3} \mathrm{CN}$ temperature is large (close to $300 \mathrm{~K}$ ) but with a large uncertainty as shown in the $\chi^{2}$ plots (see Figs. B.1-B.4). For core D, the $\mathrm{CH}_{3} \mathrm{CN}$ emission is too weak and cannot be fitted, while from the $\mathrm{CH}_{3} \mathrm{OH}$ line fitting we measure a temperature of $\sim 120 \mathrm{~K}$ (cooler than the three strongest cores). The column densities range between $1-4 \times 10^{16} \mathrm{~cm}^{-2}$ for $\mathrm{CH}_{3} \mathrm{CN}$, and $1-6 \times 10^{18} \mathrm{~cm}^{-2}$ for $\mathrm{CH}_{3} \mathrm{OH}$.
The mass of each core has been computed from

$$
\left[\frac{M_{\text {core }}}{M_{\odot}}\right]=4.597\left[\frac{S_{870 \mu \mathrm{m}}}{\mathrm{Jy}}\right]\left[\frac{D}{\mathrm{kpc}}\right]^{2}\left(\exp \left(\frac{16.46}{[T / \mathrm{K}]}\right)-1\right)
$$

with $S_{870 \mu \mathrm{m}}$ the dust continuum flux density at $870 \mu \mathrm{m}$ (see Table 2), and $D$ the distance to the source. We have adopted a dust opacity of $1.75 \mathrm{~cm}^{2} \mathrm{~g}^{-1}$ at $870 \mu \mathrm{m}$ (Ossenkopf \& Henning 1994), with a gas-to-dust ratio of 100 . We assume $T=T_{\mathrm{ex}}$, where $T_{\text {ex }}$ is obtained from the myXCLASS analysis. Since $\mathrm{CH}_{3} \mathrm{CN}$ and $\mathrm{CH}_{3} \mathrm{OH}$ may be biased towards warm gas $(>100 \mathrm{~K}$ ), but the dust may also be tracing cooler material, we have set a lower limit for the temperature of $30 \mathrm{~K}$. In Table 4, we give the range of temperatures used and the derived masses. For cores $\mathrm{E}$ and $\mathrm{F}$, without detection of hot core molecules, we assume a temperature range of $30-50 \mathrm{~K}$. The masses of the cores in G35.20N are in the range $1-10 M_{\odot}$.

We have also calculated the source-averaged column density, $N_{\text {core }}$, and volume density, $n_{\text {core }}$, under the assumption that the cores are spherically symmetric and homogeneous:

$$
\begin{aligned}
& {\left[\frac{N_{\text {core }}}{\mathrm{cm}^{-2}}\right]=2.95 \times 10^{24}\left[\frac{M_{\text {core }}}{M_{\odot}}\right]\left[\frac{\mu}{2.3}\right]^{-1}\left[\frac{\theta_{\mathrm{S}}}{\operatorname{arcsec}}\right]^{-2}\left[\frac{D}{\mathrm{kpc}}\right]^{-2}} \\
& {\left[\frac{n_{\text {core }}}{\mathrm{cm}^{-3}}\right]=2.95 \times 10^{8}\left[\frac{M_{\text {core }}}{M_{\odot}}\right]\left[\frac{\mu}{2.3}\right]^{-1}\left[\frac{\theta_{\mathrm{S}}}{\operatorname{arcsec}}\right]^{-3}\left[\frac{D}{\mathrm{kpc}}\right]^{-3},}
\end{aligned}
$$

where $\mu=2.3$ is the mean molecular weight, and $\theta_{\mathrm{S}}$ is the deconvolved angular diameter (see Table 2). The results are given in Table 4 . The mean column and volume densities are $1-10 \times 10^{24} \mathrm{~cm}^{-2}$ and $2-9 \times 10^{8} \mathrm{~cm}^{-3}$, with core A being the densest. From these values and the $\mathrm{CH}_{3} \mathrm{CN}$ and $\mathrm{CH}_{3} \mathrm{OH}$ column densities (see Table 3), we derive a $\mathrm{CH}_{3} \mathrm{CN}$ fractional abundance of $0.10-1.1 \times 10^{-8}, 0.21-1.3 \times 10^{-8}$ and $0.27-2.0 \times 10^{-8}$, for cores $\mathrm{A}, \mathrm{B}$, and $\mathrm{C}$, respectively. For $\mathrm{CH}_{3} \mathrm{OH}$, we obtain $0.15-1.7 \times 10^{-6}, 0.45-2.6 \times 10^{-6}, 1.3-9.8 \times 10^{-6}$ and $0.11-$ $0.47 \times 10^{-6}$, for cores A, B, C, and D. These abundances ${ }^{6}$ are similar to the values found toward other hot cores associated with high-mass and intermediate-mass YSOs (e.g., Charnley et al. 1992; Fuente et al. 2005, 2009; Sánchez-Monge et al. $2010)$, and only slightly lower than the abundances $\left(\gtrsim 10^{-8}\right.$ for $\mathrm{CH}_{3} \mathrm{CN}$, and $\gtrsim 10^{-6}$ for $\mathrm{CH}_{3} \mathrm{OH}$ ) measured in the strongest hot cores (e.g., Remijan et al. 2004; Bisschop et al. 2007; Mookerjea et al. 2007; Wang et al. 2010). We also computed the sourceaveraged surface density as $\Sigma_{\text {core }}=4 M_{\text {core }} /\left(\pi D_{\mathrm{S}}^{2}\right)$, where the source diameter, $D_{\mathrm{S}}$, is taken from Table 2 .

The G35.20N region is believed to be forming high-mass stars, based on the large luminosity (see Sect. 3.3), association with maser emission, and measurements of the mass of the embedded stars (Sánchez-Monge et al. 2013b). However, from Table 4, one sees that the values of the core masses are typically small, so one may wonder whether such a limited amount of gas is consistent with the formation of a massive star. We note that, as a matter of fact, there are quite a few B-type protostars associated with cores of a few $M_{\odot}$, such as IRAS 20126+4104 (Cesaroni et al. 2005; Beltrán et al. 2011), W3 IRS5 (Wang et al. 2013) and G35.03+0.35 (Beltrán et al. 2014). It is possible that cores that "light" are only the remnants of the original envelopes enshrouding the protostars, while

\footnotetext{
6 We note that missing flux may affect differently the spatial distributions of gas and dust, introducing some uncertainties in the reported abundances.
} 


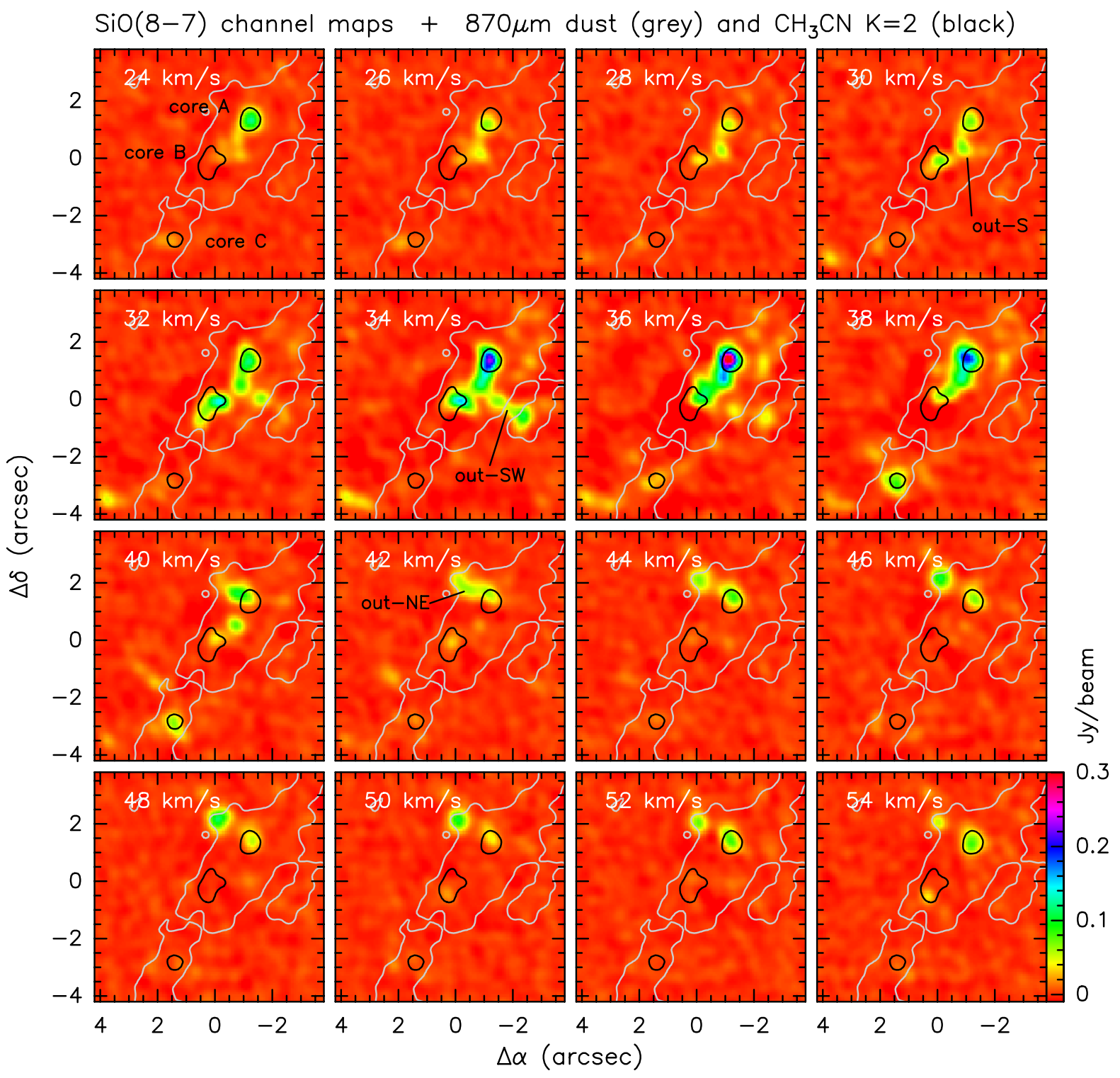

Fig. 7. $\mathrm{SiO}(8-7)$ channel map of the G35.20-0.74 N region, averaged over $2 \mathrm{~km} \mathrm{~s}^{-1}$ wide velocity intervals. The central velocity of each channel is indicated in the upper left corner, and the velocity to take as reference is $+32 \mathrm{~km} \mathrm{~s}^{-1}$. Gray contour: $5 \sigma$ level of the $870 \mu \mathrm{m}$ ALMA continuum emission shown in Fig. 1. Black contour: 50\% intensity level of the $\mathrm{CH}_{3} \mathrm{CN}(19-18) K=2$ integrated emission as shown in Figs. A.1 and 5. The structures named "out-S", "out-SW" and 'out-NE' are discussed in Sect. 3.2.3, while the spectra of these three regions are shown in Fig. 8.

Table 3. Best myXCLASS fit parameters for the $\mathrm{CH}_{3} \mathrm{CN}$ and $\mathrm{CH}_{3} \mathrm{OH}$ species (see Figs. B.1-B.4).

\begin{tabular}{|c|c|c|c|c|c|c|c|c|c|c|}
\hline \multirow[b]{2}{*}{ Core } & \multicolumn{5}{|c|}{$\mathrm{CH}_{3} \mathrm{CN}$} & \multicolumn{5}{|c|}{$\mathrm{CH}_{3} \mathrm{OH}$} \\
\hline & $\begin{array}{c}v \\
\left(\mathrm{~km} \mathrm{~s}^{-1}\right) \\
\end{array}$ & $\begin{array}{c}\Delta v \\
\left(\mathrm{~km} \mathrm{~s}^{-1}\right)\end{array}$ & $\begin{array}{c}\text { size } \\
(\operatorname{arcsec})\end{array}$ & $\begin{array}{c}T_{\mathrm{CH}_{3} \mathrm{CN}} \\
(\mathrm{K}) \\
\end{array}$ & $\begin{array}{c}N_{\mathrm{CH}_{3} \mathrm{CN}} \\
\left(\mathrm{cm}^{-2}\right) \\
\end{array}$ & $\begin{array}{c}v \\
\left(\mathrm{~km} \mathrm{~s}^{-1}\right) \\
\end{array}$ & $\begin{array}{c}\Delta v \\
\left(\mathrm{~km} \mathrm{~s}^{-1}\right)\end{array}$ & $\begin{array}{c}\text { size } \\
(\operatorname{arcsec})\end{array}$ & $\begin{array}{c}T_{\mathrm{CH}_{3} \mathrm{OH}} \\
(\mathrm{K}) \\
\end{array}$ & $\begin{array}{c}N_{\mathrm{CH}_{3} \mathrm{OH}} \\
\left(\mathrm{cm}^{-2}\right) \\
\end{array}$ \\
\hline $\mathrm{A}$ & 31.8 & 5.2 & 0.24 & 309 & $3.8 \times 10^{16}$ & 31.8 & 4.0 & 0.34 & 192 & $6.0 \times 10^{18}$ \\
\hline B & 29.6 & 2.8 & 0.31 & 148 & $1.0 \times 10^{16}$ & 29.9 & 2.9 & 0.36 & 177 & $2.1 \times 10^{18}$ \\
\hline $\mathrm{C}$ & 32.4 & 8.1 & 0.12 & 280 & $1.0 \times 10^{16}$ & 32.0 & 5.4 & 0.09 & 176 & $4.9 \times 10^{18}$ \\
\hline D & $\ldots$ & $\ldots$ & $\ldots$ & $\ldots$ & $\ldots$ & 31.2 & 1.5 & 0.07 & 120 & $0.8 \times 10^{18}$ \\
\hline
\end{tabular}

most of the material has already been accreted and/or dispersed. As suggested by Cesaroni (2005), the "light" cores might be forming only one or very few massive stars, unlike the "heavy" cores $>100 M_{\odot}$, which likely contain multiple high-mass stars. The surface densities measured toward the cores in G35.20N (see Table 4) are significantly higher than $0.15-1 \mathrm{~g} \mathrm{~cm}^{-2}$, which is the theoretical minimum value needed to form massive stars (Krumholz \& McKee 2008; Butler \& Tan 2012). We note, however, that the theoretical minimum surface density usually refers to clumps or clouds larger than the cores detected in G35.20N. We also compared the mass and size of the six cores identified in $\mathrm{G} 35.20 \mathrm{~N}$ with the empirical relation for a dense core to form 


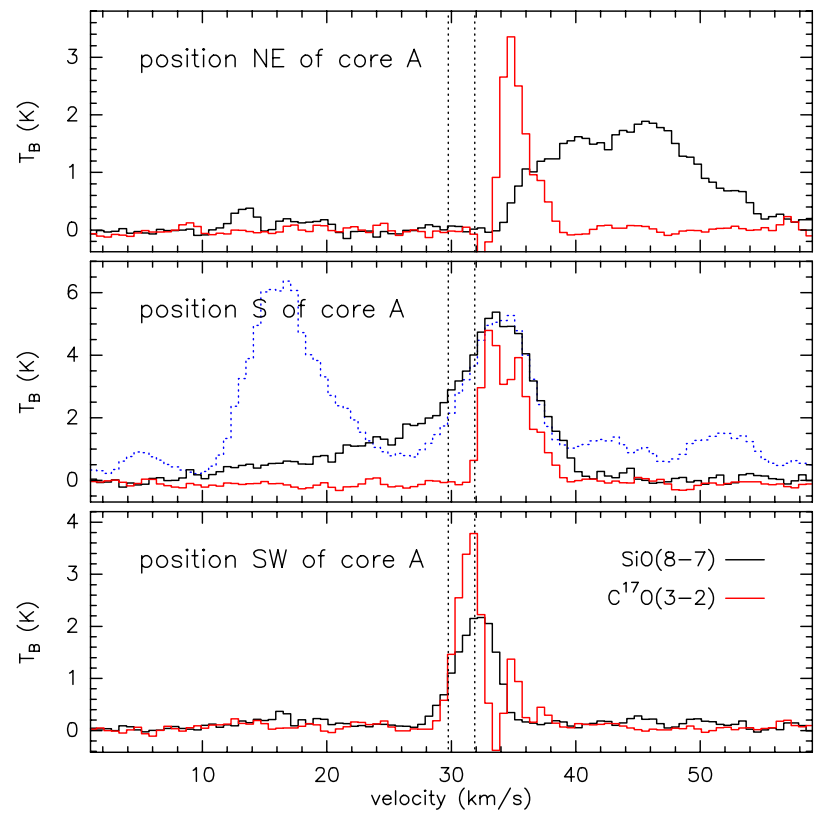

Fig. 8. Spectra of the $\mathrm{SiO}(8-7)$ emission (black line) toward different positions in the G35.20N star forming region: positions "out-NE" (top), "out-S" (middle) and "out-SW" (bottom) as indicated in Fig. 7. The emission has been obtained by integrating over areas of 1.34, 0.45 and $1.19 \operatorname{arcsec}^{2}$, which yield to conversion factors of 8.5, 25.3 and $9.6 \mathrm{~K} \mathrm{Jy}^{-1}$, respectively for the three panels. The red line corresponds to the $\mathrm{C}^{17} \mathrm{O}(3-2)$ line emission toward the three positions. In the middle panel, the blue dotted line corresponds to the $\mathrm{SiO}(8-7)$ line emission at the position of core A (over an area of $1.37 \mathrm{arcsec}^{2}$, which yields to a conversion factor of $8.9 \mathrm{~K} \mathrm{Jy}^{-1}$ ). The velocity component at $\sim 16 \mathrm{~km} \mathrm{~s}^{-1}$ corresponds to two blended $\mathrm{CH}_{3} \mathrm{CHO}$ transitions. The two vertical dotted lines indicate the velocities of core A $\left(31.8 \mathrm{~km} \mathrm{~s}^{-1}\right)$ and core B $\left(29.6 \mathrm{~km} \mathrm{~s}^{-1}\right)$.

a massive star as derived by Kauffmann \& Pillai (2010, see also Elia et al. 2013). In Fig. 11, we see that the six cores as well as the whole elongated structure ${ }^{7}$ lay above the mass-size relation. However, we note that this relation is derived for clumps/clouds with sizes $0.1-10 \mathrm{pc}$, and it might be no longer a valid description for cores with sizes $\sim 0.01 \mathrm{pc}$. Further observations are thus required to confirm if cores D to F may form high-mass stars, or only intermediate/low-mass counterparts.

Finally, we note that the mass of the whole elongated structure derived by us is several times less than that of $\sim 130 \mathrm{M}_{\odot}$ estimated by Gibb et al. (2003) and Birks et al. (2006) at lower angular resolution, assuming a dust temperature of $33 \mathrm{~K}$ and the opacities of Ossenkopf \& Henning (1994). This indicates that our ALMA observations resolve out part of the large-scale, lower-density envelope, and are only sensitive to more compact and dense material, as suggested from the missing flux estimation done in Sect. 3.1. We also note that a fraction of the mass available in the elongated structure might be eventually accreted onto the different dense cores, and increase the final mass of the stars forming there.

\footnotetext{
7 The mass of the whole elongated structure imaged with ALMA has been computed by adding the masses of the cores to the mass of the extended emission. The flux density of the whole structure is $\sim 3.3 \mathrm{Jy}$, with $\sim 2$ Jy coming from the cores (see Table 2). We assume that the remaining flux $(\sim 1.3 \mathrm{Jy})$ comes from gas with a temperature of 30 $50 \mathrm{~K}$ to derive a total mass of the structure (extended emission plus dense cores) of $21-37 M_{\odot}$.
}

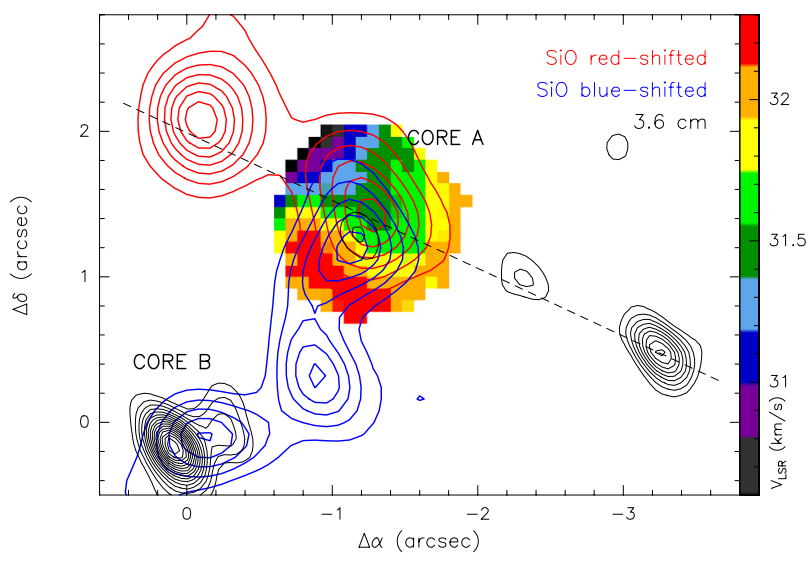

Fig. 9. Maps of the blue- (blue contours) and red-shifted (red contours) $\mathrm{SiO}(8-7)$ emission overlaid on the $\mathrm{CH}_{3} \mathrm{CN}(19-18) K=2$ velocity field (color scale) towards core A. The maps have been obtained by averaging the emission over the velocity intervals $25.2-28.6 \mathrm{~km} \mathrm{~s}^{-1}$ and $40.8-57.7 \mathrm{~km} \mathrm{~s}^{-1}$. Contour levels range from 0.05 to 0.27 in steps of $0.05 \mathrm{Jy} \mathrm{beam}^{-1} \mathrm{~km} \mathrm{~s}^{-1}$ and from 0.15 to 1.19 in steps of $0.15 \mathrm{Jy} \mathrm{beam}^{-1} \mathrm{~km} \mathrm{~s}^{-1}$, for blue and red-shifted emission, respectively. Black contours are a map of the $3.6 \mathrm{~cm}$ continuum emission (Gibb et al. 2003), with levels from 0.039 to 0.533 in steps of $0.026 \mathrm{mJy} \mathrm{beam}^{-1}$. The dashed line shows the direction of a possible jet associated with core A.

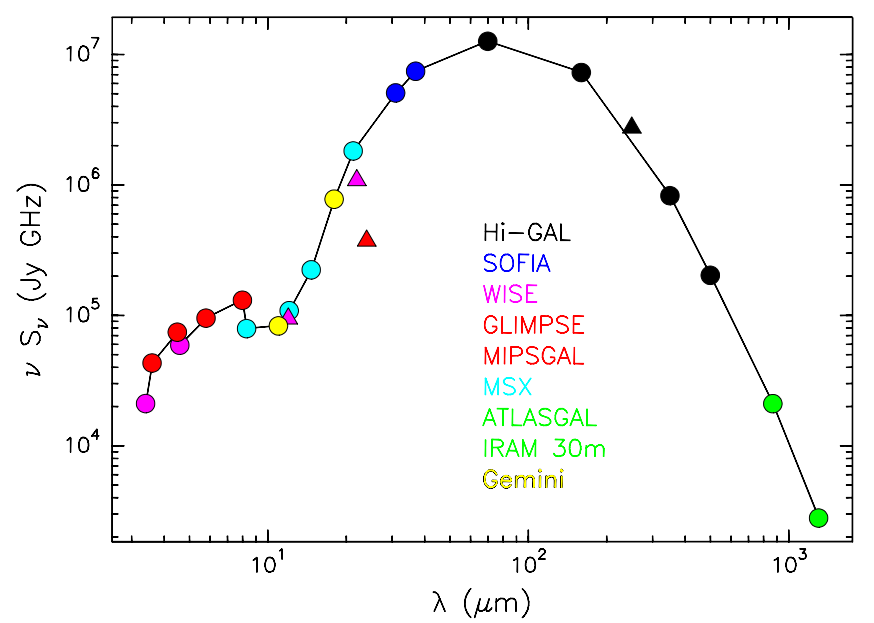

Fig. 10. Continuum spectrum of the G35.20N star forming region. Triangles denote lower limits. Different colors correspond to different surveys and instruments, as indicated in the figure.

\subsection{Dynamical status of the cores}

We estimated the virial masses of the cores identified in Fig. 1 as $M_{\text {vir }}=105\left[D_{\mathrm{S}} / \mathrm{pc}\right]\left[\Delta v / \mathrm{km} \mathrm{s}^{-1}\right]^{2} M_{\odot}$ (e.g., MacLaren et al. 1988; Bertoldi \& McKee 1992), where $D_{\mathrm{S}}$ is the diameter of the core from Table $2, \Delta v$ is the line full width at half maximum (FWHM), and the numerical value accounts for a uniform density distribution across the core. Different numerical factors have to be considered for non-uniform density distributions, e.g., 95 for $n \propto r^{-1}$, and 63 for $n \propto r^{-2}$ (MacLaren et al. 1988). This results in a virial mass smaller by a factor 1.1 and 1.7 , respectively. The value of $\Delta v$ is obtained from an average of the $\mathrm{CH}_{3} \mathrm{CN}$ and $\mathrm{CH}_{3} \mathrm{OH}$ spectral line fits (see Table 3 ). The virial mass derived from these hot core tracers is listed in Col. 8 of Table 4 . An estimate of the virial mass is also obtained by using the $\mathrm{H}^{13} \mathrm{CO}^{+}$ 
Table 4. Physical parameters of the cores detected at $870 \mu \mathrm{m}$ (see Fig. 1).

\begin{tabular}{cccccccccc}
\hline \hline & $\begin{array}{c}T_{\text {rot }}{ }^{2} \\
\text { Core }\end{array}$ & $\begin{array}{c}M_{\text {core }}{ }^{b} \\
\left(M_{\odot}\right)\end{array}$ & $\begin{array}{c}N_{\text {core }}^{b} \\
\left(10^{24} \mathrm{~cm}^{-2}\right)\end{array}$ & $\begin{array}{c}n_{\text {core }}{ }^{b} \\
\left(10^{8} \mathrm{~cm}^{-3}\right)\end{array}$ & $\begin{array}{c}\sum_{\text {core }}^{b} \\
\left(\mathrm{~g} \mathrm{~cm}^{-2}\right)\end{array}$ & $\begin{array}{c}\Delta v^{\text {hot-core }} \\
\left(\mathrm{km} \mathrm{s}^{-1}\right)\end{array}$ & $\begin{array}{c}M_{\text {virial }}^{\text {hote }} \\
\left(M_{\odot}\right)\end{array}$ & $\begin{array}{c}\Delta v^{\mathrm{H}^{13} \mathrm{CO}^{+}} \\
\left(\mathrm{km} \mathrm{s}^{-1}\right)\end{array}$ & $\begin{array}{c}M_{\text {virial }}^{\mathrm{H}^{13} \mathrm{CO}^{+}} \\
\left(M_{\odot}\right)\end{array}$ \\
\hline A & $30-250$ & $11.2-1.0$ & $40.2-3.6$ & $44.3-4.0$ & $156.0-14.0$ & 4.6 & 9.7 & $>3.2$ & $>4.7$ \\
B & $30-150$ & $5.4-0.9$ & $4.7-0.8$ & $2.6-0.4$ & $18.9-3.1$ & 2.9 & 7.7 & $>2.9$ & $>7.7$ \\
C & $30-200$ & $3.1-0.4$ & $3.7-0.5$ & $2.4-0.3$ & $13.7-1.8$ & 6.8 & 37.7 & $>2.6$ & $>5.5$ \\
D & $30-100$ & $3.7-0.9$ & $7.0-1.7$ & $5.6-1.4$ & $24.8-6.0$ & 1.5 & 1.5 & $>2.9$ & $>5.6$ \\
E & $30-50$ & $2.6-1.4$ & $2.3-1.2$ & $1.2-0.7$ & $8.2-4.4$ & $\ldots$ & $\ldots$ & $>1.8$ & $>3.1$ \\
F & $30-50$ & $4.5-2.4$ & $2.0-1.1$ & $0.8-0.4$ & $7.5-4.0$ & $\ldots$ & $\ldots$ & $>1.0$ & $>1.3$ \\
\hline
\end{tabular}

Notes. ${ }^{(a)}$ Temperature derived from the rotational diagram method when possible (see Sect. 4.1). ${ }^{(b)}$ Mass, $M_{\text {core }}$, column density, $N_{\text {core }}$, volume density, $n_{\text {core }}$, and surface density, $\Sigma_{\text {core }}$, estimated using a dust temperature equal to $T_{\text {rot }}$, and a dust opacity of $1.75 \mathrm{~cm}^{2} \mathrm{~g}^{-1}$ at $870 \mu \mathrm{m}$ (see Sect. 4.1).

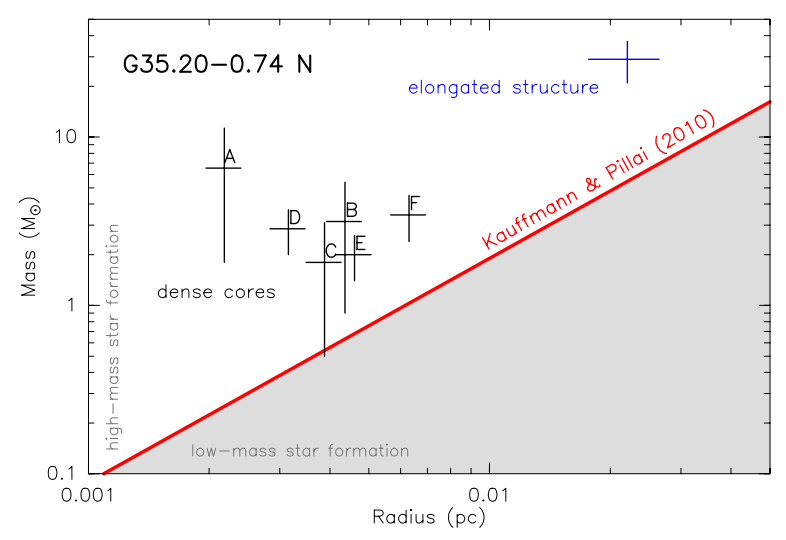

Fig. 11. Mass-radius relation for the cores (black crosses) and the elongated structure (blue cross) in G35.20N. The vertical length of the crosses denotes the uncertainty in the mass (see Table 4), while the horizontal length denotes a $10 \%$ error in the radius of the core (Table 2). The red solid line indicate the criteria for massive star formation by Kauffmann \& Pillai (2010), with the gray area corresponding to the low-mass star formation region.

line, which is detected throughout the whole elongated structure (see Fig. 3) and hence towards all the cores. We note, however, that extended structures are heavily resolved out in our observations. This results in fake absorption features in the spectra of the cores, affecting our estimates of the line widths, which might be underestimated. In Cols. 9 and 10 of Table 4, we list the lower limits obtained for the $\mathrm{H}^{13} \mathrm{CO}^{+}$line FWHM and the corresponding virial masses.

From Table 4 one sees that the virial masses are typically greater than (or similar to) the core masses. Since the former are lower limits (as explained above), we conclude that the virial parameter is $M_{\text {virial }} / M_{\text {core }} \geq 1$, with the cores possibly confined by the external pressure. It is worth noting, that other studies (e.g., Sánchez-Monge et al. 2013c; Kauffmann et al. 2013) have found similar results for the virial parameters when studying cores at scales $\sim 0.5 \mathrm{pc}$. There is a global trend in which virial parameters close to unity are found at scales $\sim 1-10 \mathrm{pc}$, whereas greater values of the virial parameter are obtained on smaller scales ( $\sim 0.01-0.5 \mathrm{pc})$, suggesting that external pressure effects might be important mainly at small scales. These results provide some evidence in favor of the theoretical idea that on small scales, feedback from e.g., protostellar winds is sufficiently effective to keep regions out of virial equilibrium at scales below $0.5 \mathrm{pc}$.

\subsection{Velocity field of cores $A$ and $B$}

As shown in Figs. 4, A.1 and A.2, the emission of almost all molecular species reveals a velocity gradient in cores $\mathrm{A}$ and
$\mathrm{B}$, oriented $\mathrm{N}-\mathrm{S}$ in core $\mathrm{A}$ and $\mathrm{SE}-\mathrm{NW}$ (i.e., along the major axis of the hot core) in core B. The velocity shift is approximately the same $\left(\sim 3-4 \mathrm{~km} \mathrm{~s}^{-1}\right)$ for all species and transitions. In Figs. 12 and 13, we present position-velocity (PV) cuts along the direction where the velocity gradient is maximum: $\mathrm{PA}=157^{\circ}$ for core $\mathrm{B}$ and $\mathrm{PA}=10^{\circ}$ for core $\mathrm{A}$. In the following we analyze these plots separately for the two cores.

\subsubsection{Core B}

Sánchez-Monge et al. (2013b) studied the velocity pattern of core B. The authors fitted the emission of several molecules in each velocity channel with a 2D Gaussian. The positions of the Gaussian peaks, which provide a picture of the velocity field (see Fig. 4 of Sánchez-Monge et al. 2013b), were fitted with a simple Keplerian disk model. From the best fit, the authors concluded that the velocity gradient in core B traces a Keplerian disk rotating about a central mass of $18 M_{\odot}$, and inclined $19^{\circ}$ with respect to the plane of the sky. The position angle of the disk is $157^{\circ}$. Comparing the molecular disk detected with ALMA with the centimeter continuum emission (Gibb et al. 2003) and the $4.5 \mu \mathrm{m}$ Spitzer/IRAC emission (see Fig. 1a), the authors concluded that the disk in core B rotates around a central object which is the powering source of the NE-SW outflow/jet seen in the centimeter (Gibb et al. 2003), in the infrared (Dent et al. 1985b; Fuller et al. 2001; De Buizer 2006; Zhang et al. 2013) and in large-scale molecular outflow tracers (Dent et al. 1985a; Gibb et al. 2003; Birks et al. 2006).

The PV-plots towards core B (see Fig. 12) are consistent with the interpretation of Sánchez-Monge et al. (2013b), as they present a "butterfly" pattern typical of Keplerian rotation (see e.g., Cesaroni et al. 2005), with the emission extending to high velocities $\left( \pm 5 \mathrm{~km} \mathrm{~s}^{-1}\right.$ with respect to the systemic velocity of $\sim 30 \mathrm{~km} \mathrm{~s}^{-1}$ ) at small offsets from the dust continuum peak (expected to trace the star position), and small velocities $\left( \pm 1 \mathrm{~km} \mathrm{~s}^{-1}\right)$ at large offsets. The white pattern shown in the figure, encompasses the region where emission is expected for an edge-on Keplerian disk of radius 1'.2 (or $2600 \mathrm{AU}$ ), rotating about an $18 M_{\odot}$ central mass. This pattern is roughly consistent with the data, taking into account that it is obtained for zero line width and infinite angular resolution.

The PV-plots appear to change depending on the molecule. Species such as $\mathrm{CH}_{3} \mathrm{CN}, \mathrm{HC}_{3} \mathrm{~N}$ or $\mathrm{SO}_{2}$ have the emission peak to the southeast at blue-shifted velocities $\left(\sim 28 \mathrm{~km} \mathrm{~s}^{-1}\right)$, whereas species such as $\mathrm{CH}_{3} \mathrm{OH}, \mathrm{CH}_{3} \mathrm{OCHO}$, and $\mathrm{H}_{2} \mathrm{CS}$ peak to the northwest at red-shifted velocities $\left(\sim 31 \mathrm{~km} \mathrm{~s}^{-1}\right)$. We find that $\mathrm{N}$-bearing molecules are typically stronger to the southeast of the continuum peak, while O-bearing molecules are stronger to the northwest. These differences could be a consequence of 

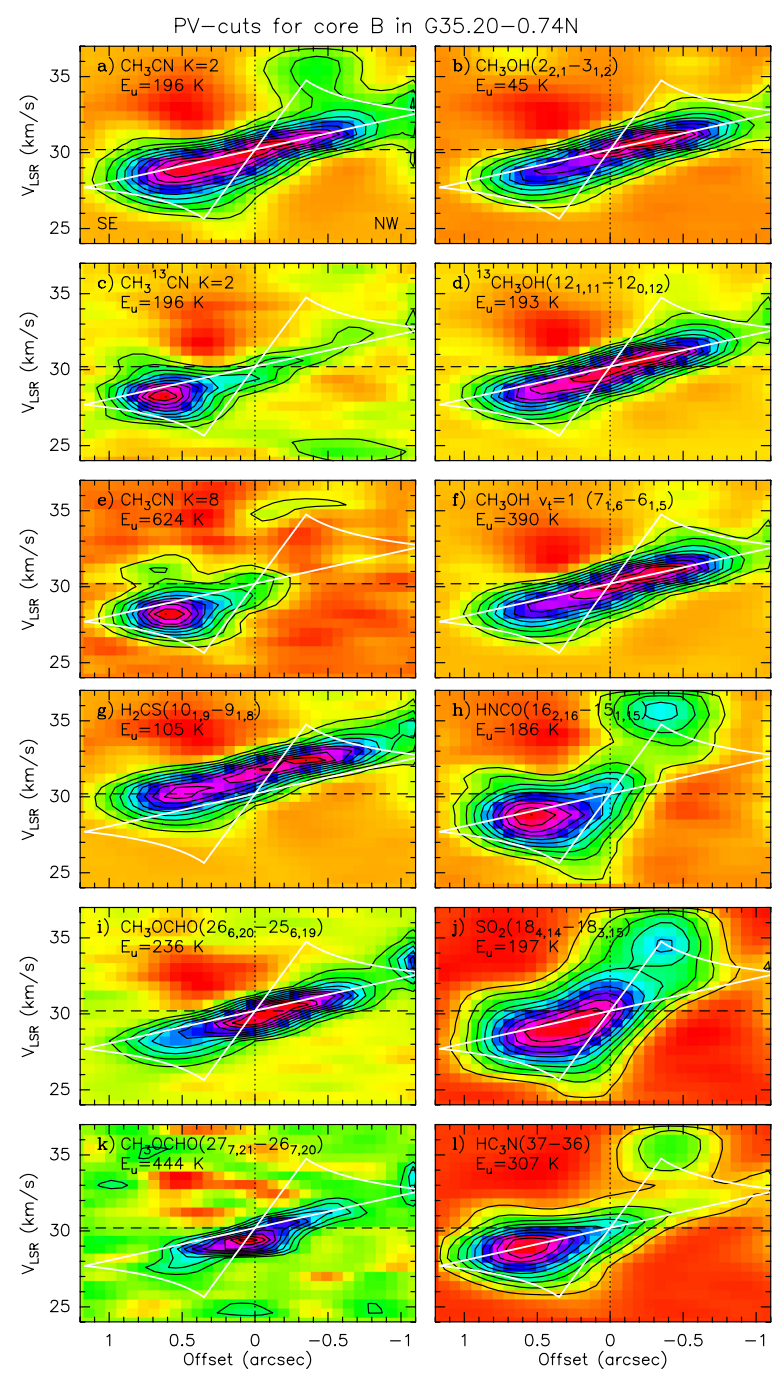

Fig. 12. Position-velocity plots along the direction with $\mathrm{PA}=157^{\circ}$ towards core B for different species and transitions. The offsets are measured from the dust peak position of core B (see Table 2), positive towards southeast. Contour levels start at $10 \%$, increasing in steps of $10 \%$ of the peak: a) 0.53, b) 0.72 , c) 0.08 , d) 0.33 , e) 0.10 , f) 0.51 , g) 0.56 , h) 0.27 , i) 0.09 , j) 0.37 , k) 0.02 and l) $0.52 \mathrm{Jy} \mathrm{km} \mathrm{s}^{-1}$. The white solid line marks the border of the region where emission is expected for an edge-on Keplerian disk of radius 1".2 (2600 AU) rotating about a $18 M_{\odot}$ central mass.

chemical variations across the disk, perhaps due to the interaction with nearby sources. An asymmetry was also observable in the Keplerian disk model fit presented in Sánchez-Monge et al. (2013b).

The Keplerian pattern is less evident in transitions like $\mathrm{CH}_{3} \mathrm{CN} \mathrm{K}=8, \mathrm{CH}_{3}{ }^{13} \mathrm{CN} K=2$ or $\mathrm{CH}_{3} \mathrm{OCHO}\left(27_{7,21}-26_{7,20}\right)$ (see Figs. 12c, e, and k), because these are detected only on one side of the disk. This suggests that not only the molecular abundance, but also the density distribution is not axially symmetric, similar to the case of the disk around the B-type protostar IRAS 20126+4104 (e.g., Cesaroni et al. 2014).

\subsubsection{Core A}

Core A is less resolved than core B, which hinders an analysis like that carried out by Sánchez-Monge et al. (2013b). The velocity gradient in this case is almost N-S. If this is tracing a rotating disk, one should see a jet/outflow perpendicular to it,
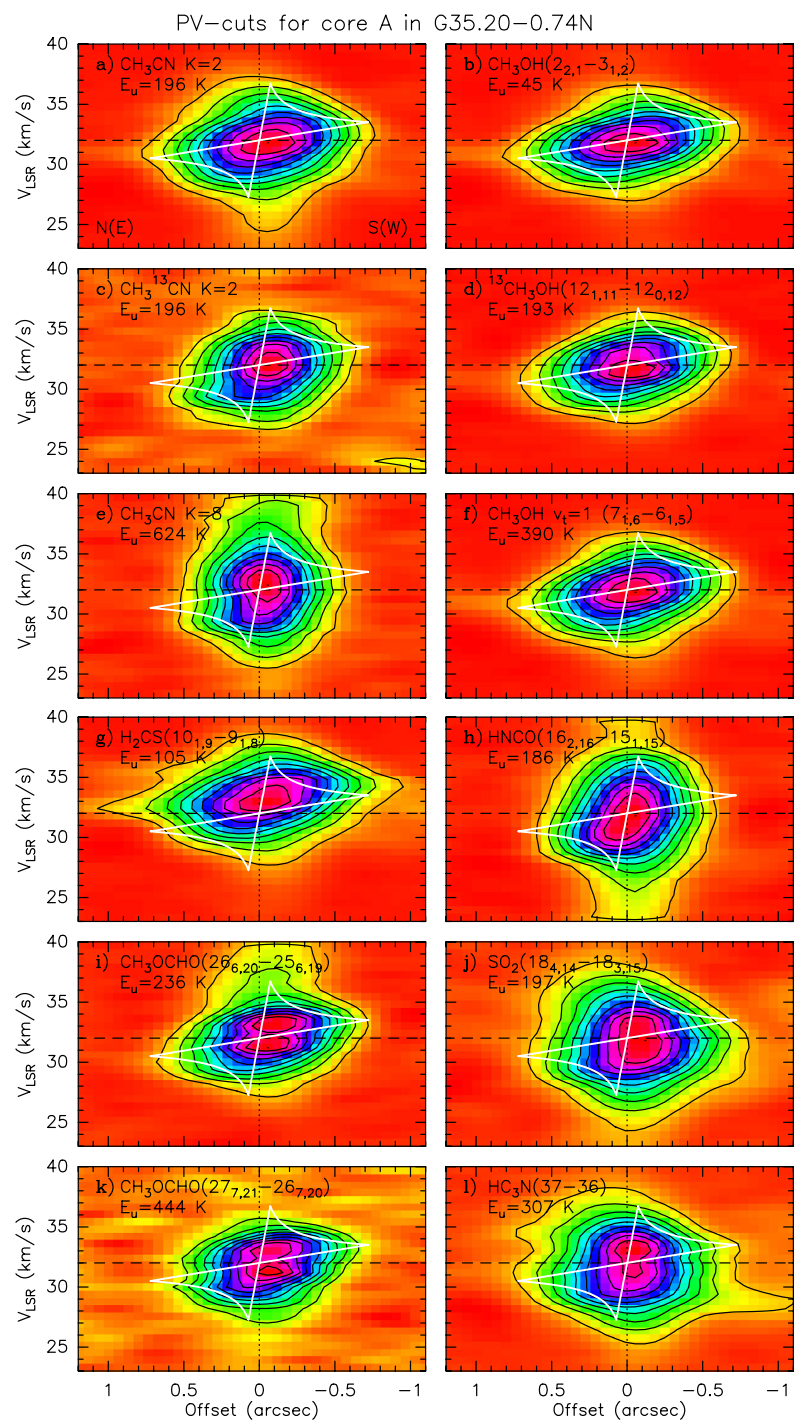

Fig. 13. Position-velocity plots along the direction with $\mathrm{PA}=10^{\circ}$ towards core A for different species and transitions. The offsets are measured from the dust peak position of core A (see Table 2), positive towards northeast. Contour levels start at $10 \%$, increasing in steps of $10 \%$ of the peak: a) 0.87 ; b) 0.99 ; c) 0.13 ; d) 0.64 ; e) 0.23 ; f) $0.81 ;$ g) 0.76 ; h) 0.47 ; i) 0.27 ; j) 0.30 ; k) 0.06 and l) $0.34 \mathrm{Jy} \mathrm{km} \mathrm{s}^{-1}$. The white solid line marks the border of the region where emission is expected for an edge-on Keplerian disk of radius 0!7 (1500 AU) rotating about a $4 M_{\odot}$ central mass.

i.e., in the E-W direction. The only clearly confirmed outflow with a similar orientation, is the one in the NE-SW direction observed with an angular resolution $\gtrsim 5^{\prime \prime}$ (e.g., Gibb et al. 2003; Birks et al. 2006). As discussed later in Sect. 4.4, this outflow is likely powered by a precessing jet arising from core B (see also Sánchez-Monge et al. 2013b). In Sect. 3.2.3, we analyzed the $\mathrm{SiO}(8-7)$ transition covered in our ALMA observations. The combination of the $\mathrm{SiO}$ emission together with the radio continuum emission (see Fig. 9) suggests the presence of a jet/outflow centered in core A and oriented approximately ENE-WSW, i.e., roughly perpendicular to the velocity gradient in the core. This lends support to the hypothesis that core A might also be a rotating disk.

The PV-plots of core A shown in Fig. 13, reveal a compact source, with a small velocity gradient. Although the evidence of Keplerian rotation for core A is less clear than that in core B, 


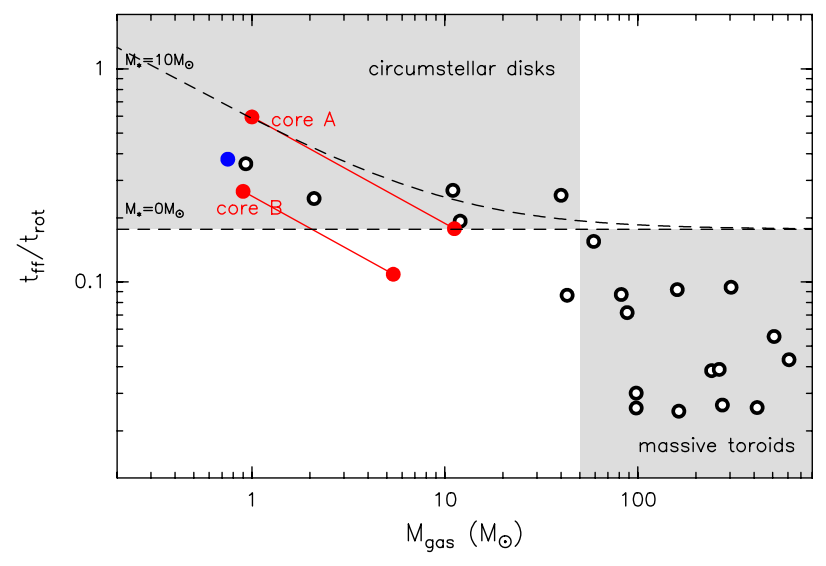

Fig. 14. Free-fall timescale to rotational period ratio $\left(t_{\mathrm{ff}} / t_{\text {rot }}\right)$ versus gas mass $\left(M_{\text {gas }}\right)$ of known rotating disks or toroids. Open circles correspond to the objects studied by Beltrán et al. (2011), blue dot corresponds to source G35.03+0.35 (Beltrán et al. 2014), and the red dots correspond to cores A and B of G35.20N (this work). The connecting line corresponds to the different gas mass estimates for different temperatures (see Table 4). The gray areas show the regions where circumstellar disks and toroids are found. The dashed lines correspond to spheres of mass $M_{\text {gas }}$, containing a star of mass $M_{*}$ (e.g., $0 M_{\odot}$ and $10 M_{\odot}$; see Sect. 4.3.3).

in a few species the pattern looks slightly elongated (see panels $\mathrm{a}, \mathrm{b}, \mathrm{f}$, and $\mathrm{g}$ ), with the emission close to the center extending up to velocities as high as $\pm 5 \mathrm{~km} \mathrm{~s}^{-1}$ (with respect to the systemic velocity of $\sim 32 \mathrm{~km} \mathrm{~s}^{-1}$ ) and the low-velocity emission $\left( \pm 1.5 \mathrm{~km} \mathrm{~s}^{-1}\right)$ detected at relatively large offsets $( \pm 0.7$ with respect to the continuum peak). Assuming that one is indeed observing a Keplerian disk with a rotation velocity of $1.5 \mathrm{~km} \mathrm{~s}^{-1}$ at a radius of 0 !' 7 ( or $1500 \mathrm{AU}$ ), we derive a mass of $\sim 4 M_{\odot}$ for the putative central star. Higher angular resolution data are needed to confirm whether core A is undergoing Keplerian rotation and derive a reliable estimate of the central mass.

\subsubsection{Comparison with other disk candidates}

In Fig. 14, we follow the approach of Beltrán et al. (2011) to compare the rotating structures found in G35.20N with those found around B-type and O-type stars. These authors plot the ratio of the free-fall timescale to the rotational period $\left(t_{\mathrm{ff}} / t_{\mathrm{rot}}\right)$ versus the gas mass $\left(M_{\mathrm{gas}}\right)$, for a sample of 20 high-mass YSOs. In this expression, $t_{\mathrm{ff}}$ is the free-fall timescale of the gas mass calculated from (sub)mm continuum emission, given by the expression $t_{\mathrm{ff}}=\sqrt{3 \pi /(32 G \rho)}$ $\left(=1.83 \times 10^{7}\left[M_{\text {gas }} / M_{\odot}\right]^{-0.5}\left[R_{\mathrm{S}} / \mathrm{pc}\right]^{1.5} \mathrm{yr}\right)$. This quantity is an estimate of the timescale of collapse of the gas under its own gravity. The rotational period, $t_{\text {rot }}=2 \pi R_{\mathrm{S}} / V_{\text {rot }}(=6.15 \times$ $\left.10^{6}\left[R_{\mathrm{S}} / \mathrm{pc}\right]\left[V_{\mathrm{rot}} / \mathrm{km} \mathrm{s}^{-1}\right]^{-1} \mathrm{yr}\right)$, is instead obtained from the observed velocity gradient and is basically the timescale needed for the disk to re-adjust its internal structure to the newly accreted material. Beltrán et al. (2011) find that rotating structures around B-type stars likely tracing circumstellar disks have higher $t_{\mathrm{ff}} / t_{\text {rot }}$ values than the massive toroids. In Fig. 14, we have added the results of cores A and B in G35.20N to the plot of Beltrán et al. (2011). In our calculations we consider a rotation velocity $V_{\text {rot }}$ of $2 \mathrm{~km} \mathrm{~s}^{-1}$ and $1.2 \mathrm{~km} \mathrm{~s}^{-1}$, and a radius of $0.01 \mathrm{pc}$ and $0.005 \mathrm{pc}$ for cores $\mathrm{B}$ and $\mathrm{A}$, respectively. The two cores of G35.20N are located in the region of Keplerian disks, which lends additional support to our hypotheses. In Fig. 14, we also show the theoretical curves corresponding to the value of $t_{\mathrm{ff}} / t_{\text {rot }}$ for a disk of mass $M_{\text {gas }}$ rotating about a star of mass $M_{*}$. This can be obtained from the previous expressions of $t_{\mathrm{ff}}$ and $t_{\mathrm{rot}}$, by substituting the rotation velocity with $V_{\text {rot }}=\sqrt{G\left(M_{\text {gas }}+M_{*}\right) / R_{\mathrm{S}}}$ :

$\frac{t_{\mathrm{ff}}}{t_{\mathrm{rot}}}=\sqrt{\frac{M_{\mathrm{gas}}+M_{*}}{32 M_{\mathrm{gas}}}}$.

The dashed line shown in the figure correspond to $M_{*}=0 M_{\odot}$ and $M_{*}=10 M_{\odot}$. From these it is clear that the objects lying in the "circumstellar disks" region are characterized by dynamically important levels of rotation, and have rotation timescales of the order (or shorter) than the dynamical timescale of collapse of the gas under its own self-gravity. Conversely, objects in the region labeled "massive toroids" are gas masses possibly supported by a combination of thermal pressure, turbulence, and magnetic fields with a little amount of rotation that however plays no role in their support. Thus, for these objects, the ratio $t_{\text {ff }} / t_{\text {rot }} t$ can take arbitrarily small values below the horizontal dashed line that corresponds to a homogeneous gas sphere in rotational equilibrium.

Finally, we estimated the velocity gradients of cores A and $\mathrm{B}$, from the velocity shifts measured for the best resolved transitions $\left(\mathrm{CH}_{3} \mathrm{CN} K=2, \mathrm{CH}_{3} \mathrm{OH}\left(7_{1,6}-6_{1,5}\right) \mathrm{H}_{2} \mathrm{CS}\left(10_{1,9}-9_{1,8}\right)\right.$ and $\left.\mathrm{HC}_{3} \mathrm{~N}(37-36)\right)$. The shifts are $\sim 4 \mathrm{~km} \mathrm{~s}^{-1}$ over an extent of $\sim 0.02 \mathrm{pc}$ for core $\mathrm{B}$, and $\sim 2.5 \mathrm{~km} \mathrm{~s}^{-1}$ over $\sim 0.01 \mathrm{pc}$ for core A (see Figs. 4-A.2 and Figs. 13 and 12), which correspond to a velocity gradient at scales $\sim 0.01 \mathrm{pc}$ of about $250 \mathrm{~km} \mathrm{~s}^{-1} \mathrm{pc}^{-1}$ and $200 \mathrm{~km} \mathrm{~s}^{-1} \mathrm{pc}^{-1}$ for cores $\mathrm{A}$ and $\mathrm{B}$, respectively. These values are consistent with the expected Keplerian velocity at a radius of $0.01 \mathrm{pc}(0.005 \mathrm{pc})$ and around a central mass of $16 M_{\odot}\left(4 M_{\odot}\right)$, for core B (core A): $\sim 2.5 \mathrm{~km} \mathrm{~s}^{-1}\left(\sim 1.8 \mathrm{~km} \mathrm{~s}^{-1}\right)$.

\subsection{Precessing jet}

As previously discussed, the velocity fields of cores A and B are suggestive of rotation about an axis oriented E-W and NE-SW, respectively. The latter direction is consistent with the axis of the bipolar nebula in Fig. 1a and corresponding outflow (see Sect. 1). This seems to confirm that the velocity gradient in core B is indeed due to rotation about a NE-SW axis. However, we know that several starforming cores are present in G35.20N, and consequently the butterfly-shaped nebula in Fig. 1a might be the result of the superposition of multiple outflows arising from these cores. Indeed, the morphology of both the mid-IR emission (see Fig. 2 of Sánchez-Monge et al. 2013b; Zhang et al. 2013) and $3.6 \mathrm{~cm}$ free-free continuum emission (Gibb et al. 2003) appears to indicate the existence of a jet from core $\mathrm{B}$ directed $\mathrm{N}-\mathrm{S}$, and hence not perpendicular to the velocity gradient in core B. Here, we argue that the non orthogonality between this jet and the core velocity gradient can be explained if the jet is precessing about an axis directed NE-SW. Little et al. (1998) were the first to suggest that such a bipolar structure could be created by a precessing jet, and Sánchez-Monge et al. (2013b) have proposed that a binary system at the center of the Keplerian disk in core B could be causing the precession.

To confirm this scenario, we have inspected the morphology of the $\mathrm{H}_{2}$ emission (a typical jet tracer) at different scales: from the scale of the core to that of the bipolar nebula, and beyond. For this purpose, we retrieved the UWISH2 image of the $\mathrm{H}_{2} 2.12 \mu \mathrm{m}$ line (Froebrich et al. 2011), shown in Fig. 15. One clearly sees that the line emission outlines a bipolar structure (dotted curves) that is the ideal extrapolation to a larger scale, of the butterflyshaped nebula seen in the IRAC $4.5 \mu \mathrm{m}$ image (white contours). 


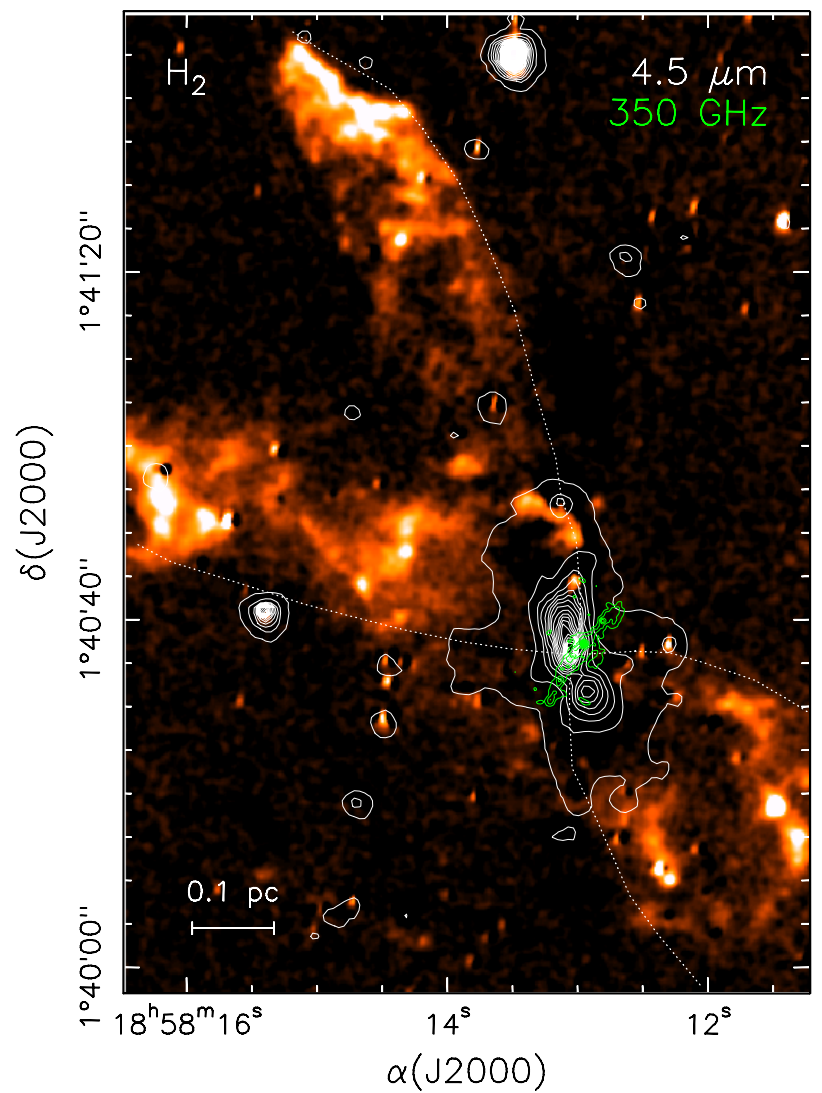

Fig. 15. Image of the $\mathrm{H}_{2} 2.12 \mu \mathrm{m}$ line emission towards G35.20. The white and green contours are, respectively, maps of the $4.5 \mu \mathrm{m}$ emission from the Spitzer/GLIMPSE survey (Benjamin et al. 2003) and $350 \mathrm{GHz}$ continuum emission from our observations. The dotted curves outline the bipolar pattern.

Even more instructive is the small-scale structure of the $\mathrm{H}_{2}$ jet illustrated in Fig. 16. Putting together the information provided by the free-free $3.6 \mathrm{~cm}$ continuum emission (cyan contours), and the $2.12 \mu \mathrm{m}$ and $4.5 \mu \mathrm{m}$ images, one sees that the jet from core B is directed $\mathrm{N}-\mathrm{S}$, up to a distance of $8^{\prime \prime}(0.085 \mathrm{pc})$ from the origin, but at that point it sharply bends to the east (see dashed curve). This behavior is strongly suggestive of precession about an axis significantly inclined with respect to the $\mathrm{N}-\mathrm{S}$ direction.

From the previous evidence we conclude that the morphology of the jet/outflow from core B is compatible with precession of the jet itself about an axis roughly perpendicular to the plane of the circumbinary disk in core B, as hypothesized by SánchezMonge et al. (2013b). We note that this interpretation does not rule out the possibility that other outflows are also present in this star forming region, as suggested by the complex structure of the $\mathrm{SiO}$ emission (see Sect. 3.2.3). Moreover, the morphology of the large-scale $\mathrm{H}_{2}$ emission seen in Fig. 15 is difficult to explain with a single precessing outflow. In particular, the branch of $\mathrm{H}_{2}$ emission extending to the east might be associated with the red lobe of the putative jet arising from core A (see Fig. 9).

\subsection{Nature of the elongated structure}

The shape and orientation of the elongated structure in Fig. 1 suggest that this might be a $2 \mathrm{D}$ flattened structure seen edgeon, lying along the waist of the bipolar nebula observed at IR wavelengths. The existence of a large-scale, rotating disk-like

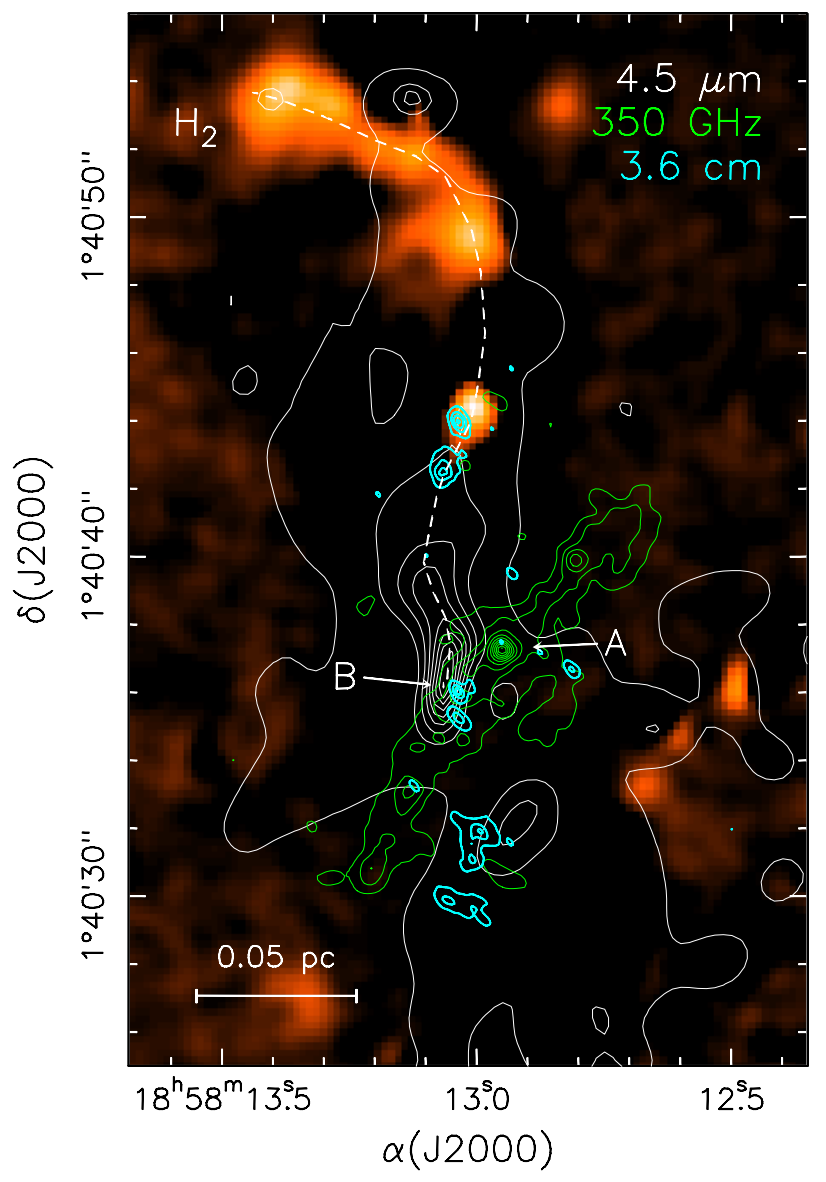

Fig. 16. Same as Fig. 15, with overlaid also the $3.6 \mathrm{~cm}$ continuum map of Gibb et al. (2003) (cyan contours). The resolution of the IRAC image (white contours) has been enhanced by HiRes deconvolution (Velusamy et al. 2008). The dashed curve outlines the shape of the jet, which is bending by almost $\sim 90^{\circ}$ at an offset of $\sim 11^{\prime \prime}$ to the north of core B.

structure has been indeed proposed by several authors. In particular, Little et al. (1985) and Brebner et al. (1987) revealed a large $\left(\sim 1^{\prime}\right.$ or $\left.0.6 \mathrm{pc}\right)$ elongated structure in $\mathrm{NH}_{3}$ and $\mathrm{CS}$, oriented SE-NW, i.e., perpendicular to the large-scale outflow, and with a velocity gradient, which they interpret as a large rotating disk/toroid. More recently, Gibb et al. (2003) studied the G35.20N star forming complex with the BIMA interferometer in the $\mathrm{H}^{13} \mathrm{CO}^{+}(1-0)$ and $\mathrm{H}^{13} \mathrm{CN}(1-0)$ transitions, with an angular resolution of $\sim 10^{\prime \prime}$. These authors find evidence of fragmentation across the same structure that they interpret as a fragmented, rotating envelope containing multiple young stellar objects, rather than a smooth rotating disk.

An alternative to the previous hypothesis, one should also consider the trivial possibility that what looks like a filament on the plane of the sky, is indeed a filament also in space. Recent continuum Herschel/Hi-GAL and spectral line observations of the interstellar medium have revealed that molecular clouds are highly filamentary (e.g., André et al. 2010; Molinari et al. 2010b; Busquet et al. 2013), which makes the filament hypothesis very plausible.

In the following we will discuss the nature of the elongated structure, confronting the two scenarios depicted above: 2D disk-like "pancake" seen edge-on, versus 1D filament.

First of all, it is important to determine the velocity field of the elongated structure, and verify whether this could be 

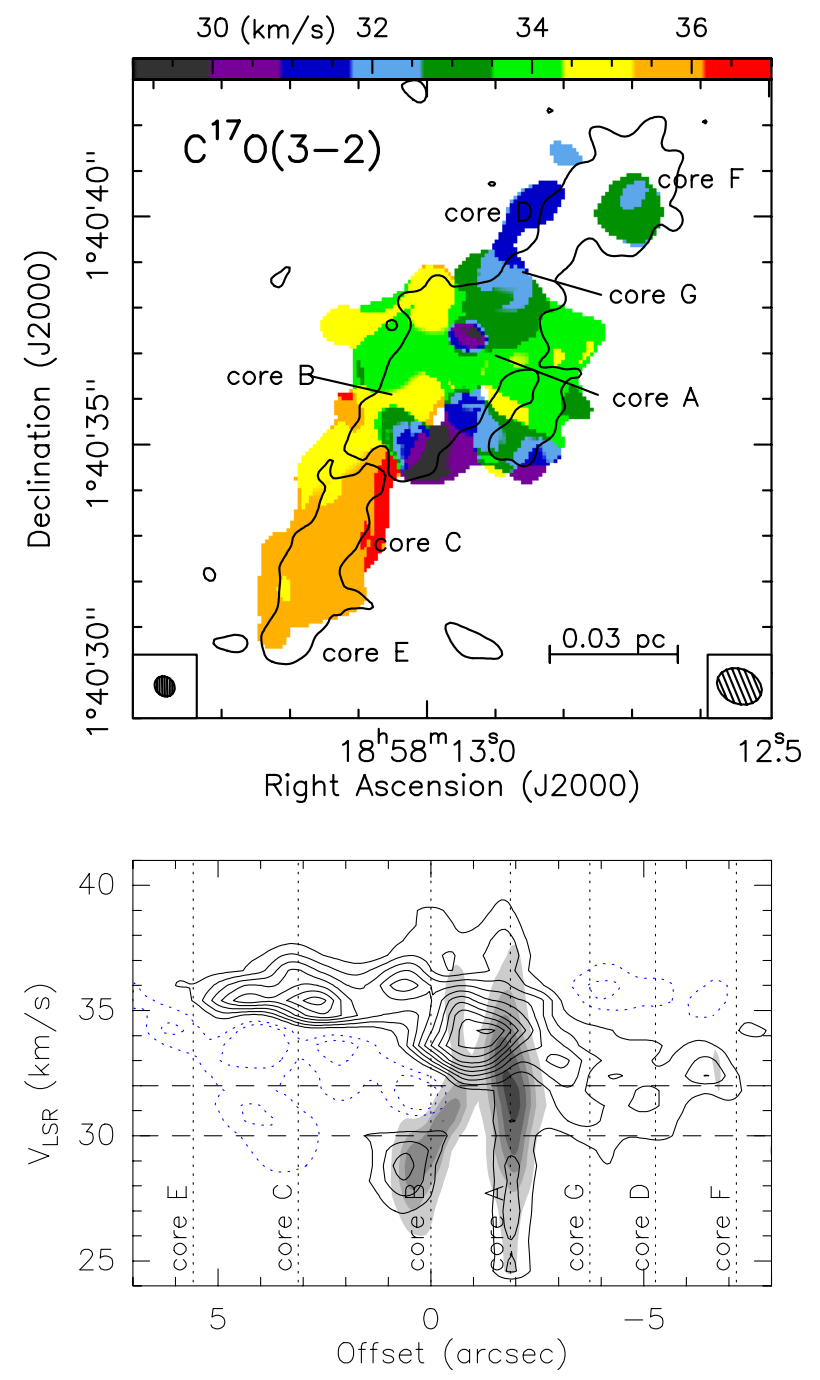

Fig. 17. Top: overlay of the $870 \mu \mathrm{m}$ continuum emission (black contour) on the velocity field (colors) of the $\mathrm{C}^{17} \mathrm{O}(3-2)$ line, after tapering the line emission at $1^{\prime \prime}$. The contour level of the continuum emission is the $9.0 \mathrm{mJy}^{\text {beam }}{ }^{-1}$ level (see Fig. 1). The velocity scale is shown in the color bar at the top of the panel, in $\mathrm{km} \mathrm{s}^{-1}$. Other symbols as in Fig. 3. Bottom: position-velocity plot along the major axis of the elongated structure $\left(\mathrm{PA}=-60^{\circ}\right)$, centered at the position of core $\mathrm{B}$, for $\mathrm{C}^{17} \mathrm{O}$ (contours) and $\mathrm{CH}_{3} \mathrm{CN}$ (gray scale). Vertical dotted lines mark the position of the cores identified in the continuum. The two horizontal dashed lines mark the velocities $30 \mathrm{~km} \mathrm{~s}^{-1}$ and $32 \mathrm{~km} \mathrm{~s}^{-1}$, associated with the hot core emission of cores $\mathrm{B}$ and $\mathrm{A}$, respectively.

rotating about the outflow axis. For this purpose we need a suitable tracer, detected all over the structure. As already shown in Fig. 3 and discussed in Sect. 3.2.1, a few molecules show extended emission which is spatially coincident with the dust $870 \mu \mathrm{m}$ continuum emission in Fig. 1. Despite the limited sensitivity of our observations to angular scales $\gtrsim 2^{\prime \prime}$, the $\mathrm{C}^{17} \mathrm{O}(3-$ 2) and $\mathrm{H}^{13} \mathrm{CO}^{+}$(4-3) transitions can be imaged toward most of the elongated structure, and may hence be used to characterize its kinematics. In the top panels of Figs. 17 and 18, we present the $870 \mu \mathrm{m}$ continuum emission map overlaid on the first order moment (velocity field) maps of the two lines. The southeastern part of the filament is associated with red-shifted emission $\left(\sim 36-37 \mathrm{~km} \mathrm{~s}^{-1}\right)$, while the central and northwestern regions have emission at velocities $\sim 31-32 \mathrm{~km} \mathrm{~s}^{-1}$. This velocity shift is more visible in the bottom panels of the two figures, which show the PV-plots of the two species along the major axis of the
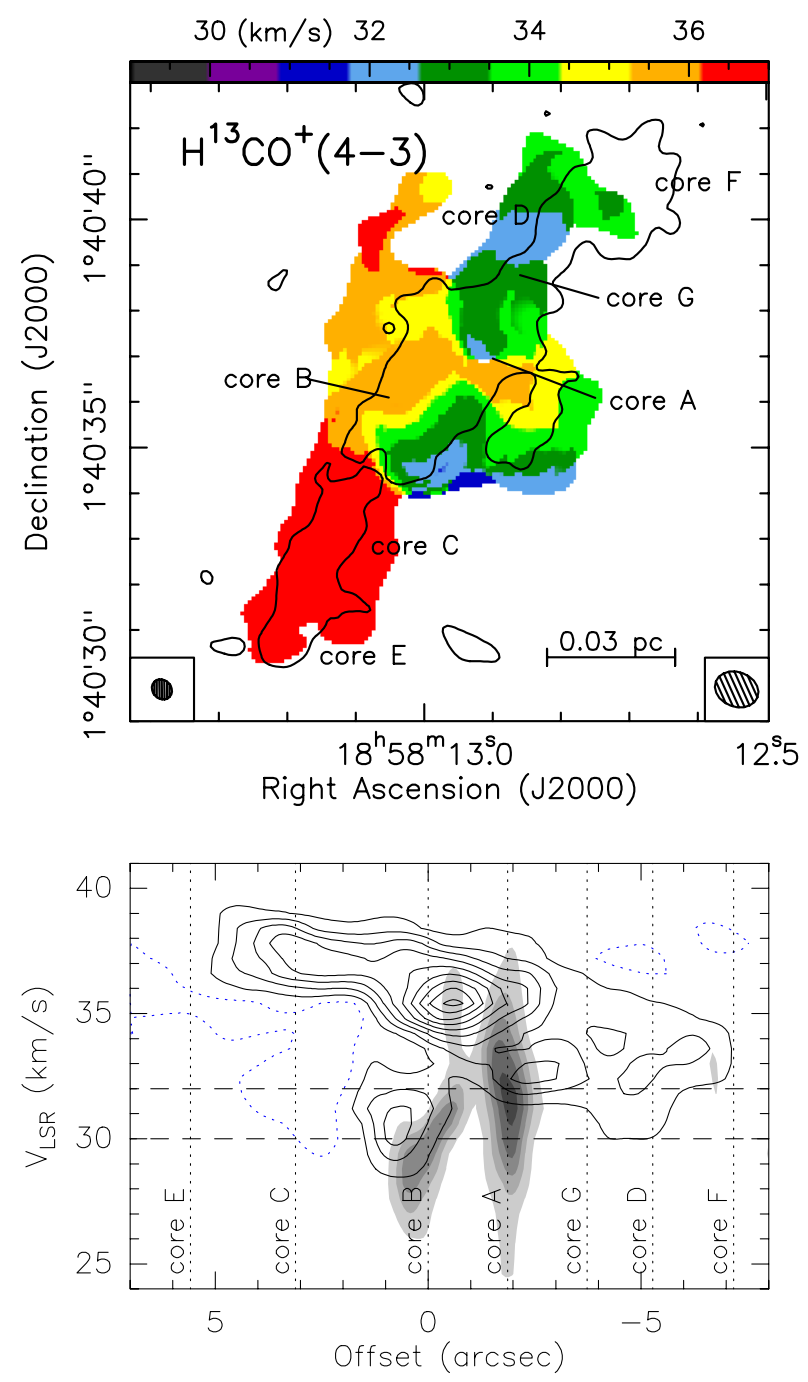

Fig. 18. As Fig. 17, but for $\mathrm{H}^{13} \mathrm{CO}^{+}(4-3)$ line.

elongated structure $\left(\mathrm{PA}=-60^{\circ}\right)$. In these plots, the velocity appears to shift gradually from $\sim 37 \mathrm{~km} \mathrm{~s}^{-1}$ close to cores $\mathrm{C}$ and $\mathrm{E}$ (southeast), to $\sim 31 \mathrm{~km} \mathrm{~s}^{-1}$ close to cores D and $\mathrm{F}$ (northwest).

The existence of a smooth velocity gradient seems to suggest that we are observing an edge-on, large-scale, rotating "pancake". Under this hypothesis one can plot the specific angular momentum as a function of distance from the center (assumed to be the position of core B), complementing our data with data from the literature (Little et al. 1985; Gibb et al. 2003; López-Sepulcre et al. 2009) that allow us to sample the putative rotating "pancake" on scales as large as $\sim 2$ pc. In Fig. 19 we show this plot, which clearly indicates that angular momentum is dissipated from the large to the small scale. Magnetic braking might be a plausible explanation of this effect, but this requires a magnetic field (B) perpendicular to the disk, whereas recent polarimetric observations (Qiu et al. 2013) suggest that the $B$ field is mainly aligned with the elongated structure. We will come back to the role of the $B$ field later on.

Another problem with the "pancake" scenario is that core B would be counter-rotating with respect to the large-scale velocity field. Even more striking is that cores A and B appear to be kinematically inconsistent with respect to the putative "pancake", as one can see by comparing the gray-scale map (hot-core tracers) to the contour map (large-scale molecular emission) in Figs. 17 
and 18. A discrepancy between the velocities of the cores and that of the structure enshrouding them is difficult to justify in a rotating "pancake", but can be explained if the two cores are created by the dynamical interaction of different filaments. Is there any evidence of such a dynamical environment around G35.20?

Figure 20 presents a large-scale $\left(\sim 5^{\prime}\right.$ or $\left.3 \mathrm{pc}\right)$ view of the G35.20N star forming region. The Spitzer/IRAC $8.0 \mu \mathrm{m}$ image (Benjamin et al. 2003) reveals a series of elongated dark features converging towards the position of G35.20N, e.g., the darkest feature, likely associated with the densest filament, towards the northwest, a curved arc-like structure towards the northeast, and two elongated structures to the south perpendicular to the main dark feature. The dust emission at $250 \mu \mathrm{m}$ (from Hi-GAL; Molinari et al. 2010a) matches the infrared dark features quite well, with the strongest emission associated with G35.20N, where the $8.0 \mu \mathrm{m}$ emission also peaks. The overall structure (panel a) resembles that of the SDC335.579-0.272 infrared dark cloud (Peretto et al. 2013), with multiple filaments converging and accreting matter towards the center, where a number of massive stars are forming. Our ALMA observations might be imaging the most prominent filament, where the accreting material is converging.

As previously noted, to discriminate an edge-on "pancake" from a filament, one could use the direction of the magnetic field, which is expected to be perpendicular to the disk plane and parallel to the filament. Recent SMA polarization measurements of the submillimeter continuum emission from G35.20N (Qiu et al. 2013) have found that the magnetic field on scales of $\sim 15^{\prime \prime}$ $(0.16 \mathrm{pc})$ is almost parallel to the elongated structure, a result that supports the filament hypothesis. However, in this case the rotation axes of cores $A$ and $B$ should also be parallel to the filament, whereas they are significantly inclined with respect to it. The direction of the rotation axes is instead consistent with the small-scale direction of the magnetic field, obtained from observations of $\mathrm{OH}$ and $\mathrm{CH}_{3} \mathrm{OH}$ masers (Hutawarakorn \& Cohen 1999; Surcis et al. 2012), which indicates that the $B$ field is oriented in the (N)E-(S)W direction (see Fig. 5 of Surcis et al. 2012) over $\sim 0.3$ (660 AU). The change of the direction of $B$ from the large to the small scale suggests that turbulence could play an important role in regulating the fragmentation of $\mathrm{G} 35.20 \mathrm{~N}$ at scales $\sim 0.1 \mathrm{pc}$. Turbulence might also explain the drastic change in the magnetic field orientation (by approximately $90^{\circ}$ ) toward the southeast of the elongated structure (see Fig. 2 by Qiu et al. 2013).

One should also establish whether a big "pancake" could be unstable against perturbations and hence produce the observed fragments. This can be investigated by estimating the Toomre stability parameter for disks, $Q$. Assuming solid-body rotation (consistent with the PV-plots in Figs. 17 and 18), this can be expressed as $Q=2 \Omega \Delta V /(\sqrt{8 \ln 2} \pi G \Sigma)$, with $\Omega$ angular velocity, $\Sigma$ surface density, and $\Delta V$ line FWHM. $\Omega \simeq 40 \mathrm{~km} \mathrm{~s}^{-1} \mathrm{pc}^{-1}$ is estimated from the slope of the PV-plots in Figs. 17 and 18, while $\Sigma$ is given by the ratio between the mass of the elongated structure $\left(30 M_{\odot}\right)$ and the disk surface $\left(\pi R^{2}\right.$, where $2 R=0.15 \mathrm{pc}$ is the length of the elongated structure). In order to obtain $Q \leq 1$, the condition for instability, the line width must be $\Delta V \leq 0.7 \mathrm{~km} \mathrm{~s}^{-1}$, a reasonable value for the initial conditions of the unperturbed gas, prior to star formation. We hence conclude that the "pancake" should break up into fragments.

The filament hypothesis may also explain the observed distribution of cores A-F. A filament (or isothermal gas cylinder) supported by "turbulence pressure" against its self-gravity has a

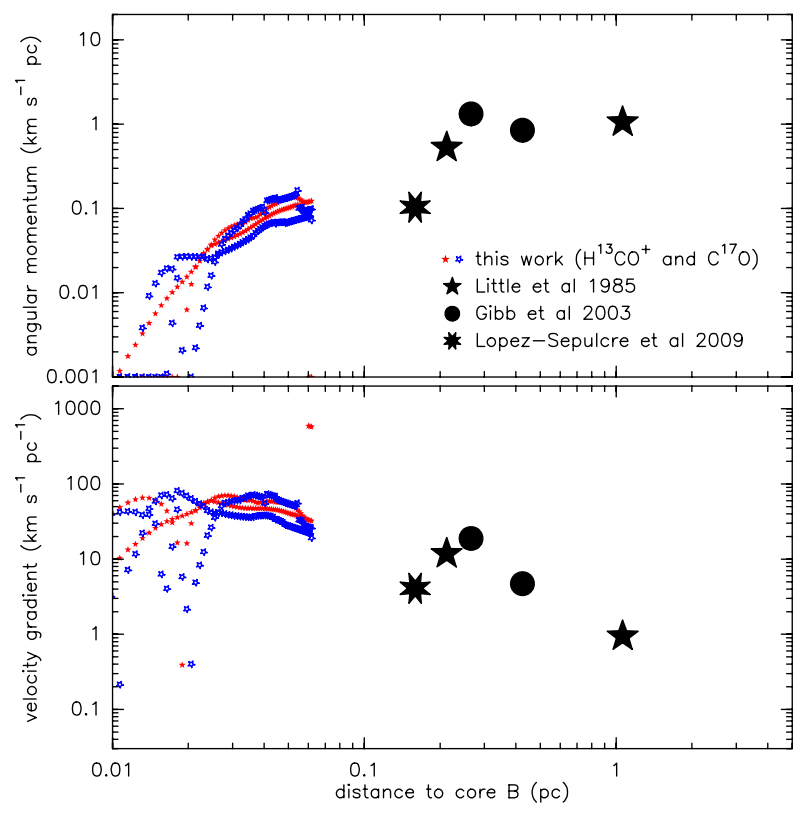

Fig. 19. Specific angular momentum (top panel) and velocity gradient (bottom panel) at different radii traced by the available molecular line data. Small red and blue stars correspond to the $\mathrm{H}^{13} \mathrm{CO}^{+}$and $\mathrm{C}^{17} \mathrm{O}$ ALMA line data presented in this work, respectively. Black fivepoint stars: $\mathrm{NH}_{3}(1,1)$ and $\mathrm{CS}(2-1)$ line data from Little et al. (1985). Black filled circles: $\mathrm{H}^{13} \mathrm{CN}(1-0)$ and $\mathrm{H}^{13} \mathrm{CO}^{+}(1-0)$ line data from Gibb et al. (2003). Black eight-point star: $\mathrm{C}^{18} \mathrm{O}(2-1)$ line data from López-Sepulcre et al. (2009).

maximum mass per unit length equal to

$(M / l)_{\text {crit }}=2 \sigma^{2} / G=465\left(\sigma / 1 \mathrm{~km} \mathrm{~s}^{-1}\right)^{2} M_{\odot} \mathrm{pc}^{-1}$

(Nagasawa 1987; Wang et al. 2014), over which it starts to fragment. The theoretical separation of the fragments is given by the expression

$\lambda=9.39 \frac{\Delta V}{\sqrt{4 \pi G \rho}}$

where $\Delta V$ is the line FWHM due to both turbulent at the thermal broadening, and relates to the velocity dispersion by $\Delta V=$ $\sigma \sqrt{8 \ln 2}$. The line width to be used in these equations should be the initial value, before fragmentation, and not the current value, measured by us, which is likely to be broadened by additional turbulence injected by the newly formed stars. In practice, one sees that a value of $\Delta V=0.7 \mathrm{~km} \mathrm{~s}^{-1}$ is required for the theoretical expression to match the observed spacing between the cores $(\sim 0.023 \mathrm{pc})$, after considering a filament with a mass $\sim 30 \mathrm{M}_{\odot}$, a size $0.15 \mathrm{pc} \times 0.013 \mathrm{pc}$, and a gas density of $\rho \simeq 10^{-16} \mathrm{~g} \mathrm{~cm}^{-3}$. In addition, this line width of $0.7 \mathrm{~km} \mathrm{~s}^{-1}$ results in a critical mass per unit length of $\sim 40 M_{\odot} \mathrm{pc}^{-1}$, while the measured mass per unit length in our filament is $\sim 200 M_{\odot} \mathrm{pc}^{-1}$. Such an initial value of $\Delta V$ is plausible and we conclude that the filament should undergo fragmentation. Similar structures undergoing fragmentation have been found in other star forming regions such as G11.11-0.12 and G28.34+0.06 (Wang et al. 2011, 2014).

Based on these results, both a rotating 'pancake' and a filament appear to be unstable and hence consistent with the observed clumpiness. However, one should take into account also an intriguing feature of the elongated structure, namely the regular spacing between the cores. The distance between two adjacent cores lies between $0.020 \mathrm{pc}$ and $0.036 \mathrm{pc}$, quite a narrow 


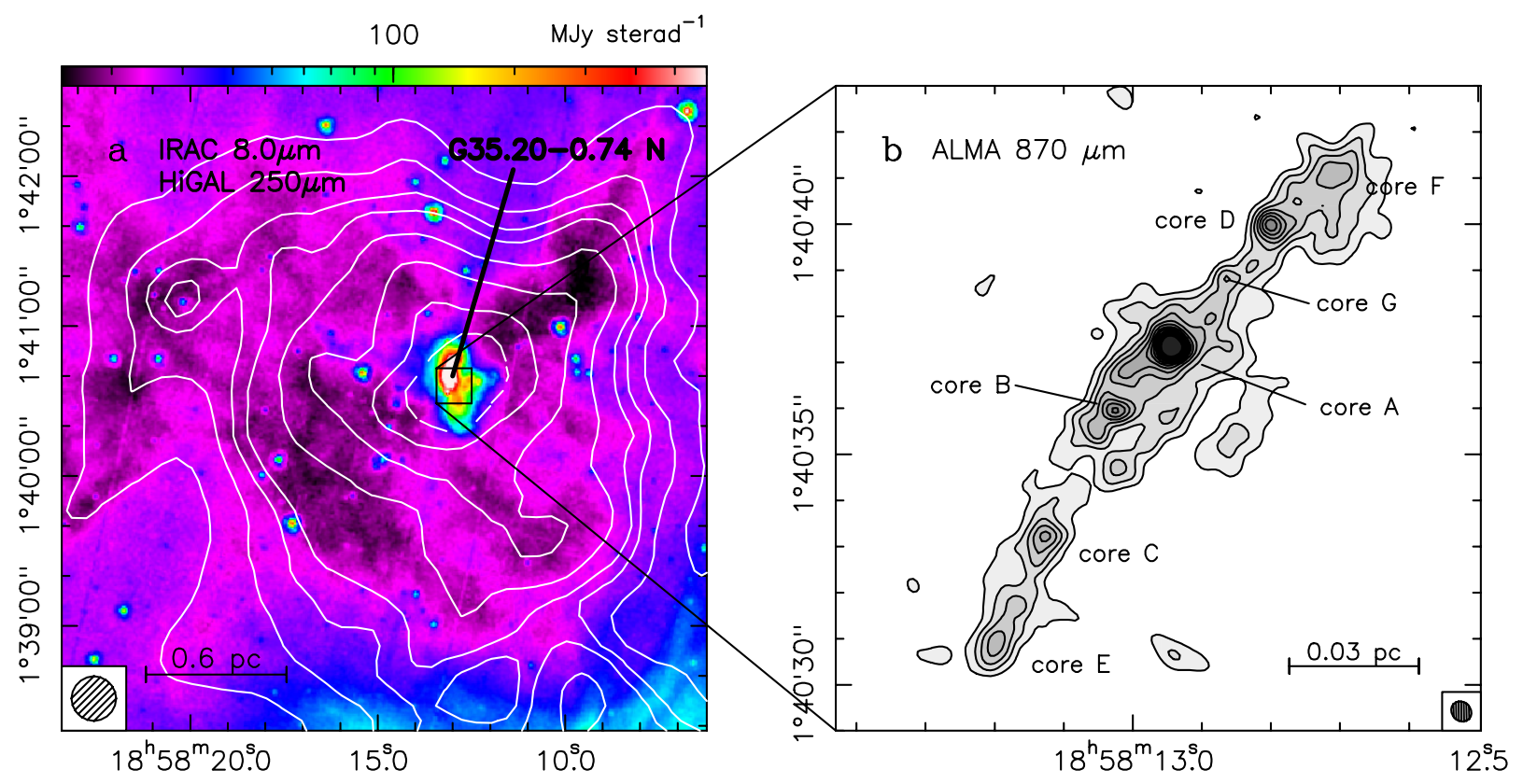

Fig. 20. a) Overlay of the Hi-GAL $250 \mu$ m continuum emission (white contours) on the Spitzer/IRAC $8.0 \mu$ m image (colors) of the G35.20-0.74 region. Contour levels start at $65 \mathrm{mJy} \mathrm{beam}^{-1}$, increasing in steps of $0.39 \mathrm{Jy}_{\text {beam }}^{-1}$ until $1.885 \mathrm{Jy} \mathrm{beam}^{-1}$, then levels increase in steps of $1.98 \mathrm{Jy}$ beam $^{-1}$ until the peak: $12 \mathrm{Jy} \mathrm{beam}^{-1}$. b) Same as Fig. $1 \mathrm{~b}$.

range of values. It seems unlikely that a random $2 \mathrm{D}$ distribution of fragments over a "pancake" can produce such a regular distribution, when seen in an edge-on projection on the plane of the sky. In conclusion, for this reason and the orientation of the magnetic field, we tend to favor the filament interpretation of the elongated structure.

\section{Summary}

We have observed the G35.20N star forming region with ALMA at $870 \mu \mathrm{m}(350 \mathrm{GHz})$, achieving an angular resolution $\sim 0$.' $4^{\prime}$ $(\sim 900 \mathrm{AU}$ at $2.19 \mathrm{kpc})$, and covering a broad frequency range including dense gas (e.g., $\mathrm{H}^{13} \mathrm{CO}^{+}, \mathrm{C}^{17} \mathrm{O}$ ), outflow (e.g., $\mathrm{SiO}$ ) and hot core (e.g., $\left.\mathrm{CH}_{3} \mathrm{CN}, \mathrm{CH}_{3} \mathrm{OH}\right)$ tracers. Our conclusions can be summarized as follows:

- The continuum emission shows an elongated dust structure (length $\sim 0.15 \mathrm{pc}$ and width $\sim 0.013 \mathrm{pc}$ ), likely tracing the densest part of the elongated structure observed at larger scales, and perpendicular to the infrared and molecular largescale outflow (e.g., Gibb et al. 2003). This structure is fragmented in six identified dense cores, although additional substructure is seen. The masses of fragments are between 1-10 $M_{\odot}$, and have sizes of around 1600 AU. The cores appear regularly spaced with a mean separation of $\sim 0.023 \mathrm{pc}$.

- Three out of the six dense cores show strong emission in complex organic molecules typical of hot cores. We fitted a large number of methyl cyanide $\left(\mathrm{CH}_{3} \mathrm{CN}\right)$ and methanol $\left(\mathrm{CH}_{3} \mathrm{OH}\right)$ spectral lines and derived temperatures of around $150-250 \mathrm{~K}$ for the three cores and relative abundances of $0.2-2 \times 10^{-8}$ for $\mathrm{CH}_{3} \mathrm{CN}$ and $0.6-5 \times 10^{-6}$ for $\mathrm{CH}_{3} \mathrm{OH}$.

- The two densest and most chemically-rich cores (cores A and B) show a coherent velocity field with a velocity gradient almost aligned with the dust elongated structure. Interestingly, the orientation of both velocity gradients is opposite, with red-shifted emission toward the north for core B and toward the south for core A. These velocity gradients are consistent with Keplerian disks rotating about central masses of $4 M_{\odot}$ for core A and $18 M_{\odot}$ for core B, i.e., intermediate/high-mass stars.

- Core B seems to power a precessing jet/outflow oriented in the NE-SW direction. The combination of the IRAC $4.5 \mu \mathrm{m}$, the UWISH2 $\mathrm{H}_{2} 2.12 \mu \mathrm{m}$ and the VLA $3.6 \mathrm{~cm}$ images together with our ALMA continuum and line maps suggests that the outflow/jet associated with core B is undergoing precession, in agreement with the binary system hypothesized by Sánchez-Monge et al. (2013b). Our ALMA high-velocity $\mathrm{SiO}(7-6)$ map together with the VLA $3.6 \mathrm{~cm}$ continuum map suggest that core A might be powering an outflow in the east-west direction. Further observations of outflow/shock tracers are required to confirm this scenario.

- The emission of dense gas tracers such as $\mathrm{H}^{13} \mathrm{CO}^{+}$and $\mathrm{C}^{17} \mathrm{O}$ is also extended and coincident with the dust emission. This large molecular structure is consistent, in morphology and velocity, with the SMA maps reported by Qiu et al. (2013). Based on the velocity field, the orientation of the magnetic field (as reported by Qiu et al. 2013), and the regularly spaced fragmentation $(\sim 0.023 \mathrm{pc})$, we interpret the elongated dust structure as the densest part of a 1D filament fragmenting and forming high-mass stars.

Acknowledgements. We are grateful to the Italian ARC node for the usage of their computer facilities during the cleaning and imaging process. We are also grateful to Ray Furuya for providing us the ASTE spectrum, and to Keping Qiu for providing the SMA spectra. Á.S.-M. is grateful to Peter Schilke, Thomas Möller and Alexander Zernickel for helping with the analysis of the myXCLASS software. This paper makes use of the following ALMA data: ADS/JAO.ALMA\#2011.0.00275.S. ALMA is a partnership of ESO (representing its member states), NSF (USA) and NINS (Japan), together with NRC (Canada) and NSC and ASIAA (Taiwan), in cooperation with the Republic of Chile. The Joint ALMA Observatory is operated by ESO, AUI/NRAO and NAOJ. This publication makes use of data products from the Widefield Infrared Survey Explorer, which is a joint project of the University of 
California, Los Angeles, and the Jet Propulsion Laboratory/California Institute of Technology, funded by the National Aeronautics and Space Administration.

\section{References}

André, P., Men'shchikov, A., Bontemps, S., et al. 2010, A\&A, 518, L102 Anglada, G. 1996, in Radio Emission from the Stars and the Sun, eds. A. R. Taylor, \& J. M. Paredes, ASP Conf. Ser., 93, 3

Beltrán, M. T., Cesaroni, R., Neri, R., \& Codella, C. 2011, A\&A, 525, A151 Beltrán, M.T., Sánchez-Monge, A., Cesaroni, R., et al. 2014, A\&A, submitted Benjamin, R. A., Churchwell, E., Babler, B. L., et al. 2003, PASP, 115, 953 Bernst, I., Schilke, P., Moeller, T., et al. 2011, in Astronomical Data Analysis Software and Systems XX, eds. I. N. Evans, A. Accomazzi, D. J. Mink, \& A. H. Rots, ASP Conf. Ser., 442, 505

Bertoldi, F., \& McKee, C. F. 1992, ApJ, 395, 140

Birks, J. R., Fuller, G. A., \& Gibb, A. G. 2006, A\&A, 458, 181

Bisschop, S. E., Jørgensen, J. K., van Dishoeck, E. F., \& de Wachter, E. B. M. 2007, A\&A, 465, 913

Boley, P. A., Linz, H., van Boekel, R., et al. 2013, A\&A, 558, A24

Bonnell, I. A., \& Bate, M. R. 2006, MNRAS, 370, 488

Brebner, G. C., Heaton, B., Cohen, R. J., \& Davies, S. R. 1987, MNRAS, 229, 679

Briggs, D. 2005, Ph.D. Thesis, New Mexico Inst. of Mining and Technology Busquet, G., Zhang, Q., Palau, A., et al. 2013, ApJ, 764, L26

Butler, M. J., \& Tan, J. C. 2012, ApJ, 754, 5

Carey, S. J., Noriega-Crespo, A., Mizuno, D. R., et al. 2009, PASP, 121, 76

Carrasco-González, C., Galván-Madrid, R., Anglada, G., et al. 2012, ApJ, 752, L29

Cesaroni, R. 2005, in Massive Star Birth: a Crossroads of Astrophysics, eds. R. Cesaroni, M. Felli, E. Churchwell, \& M. Walmsley (Cambridge University Press), IAU Symp. 227, 59

Cesaroni, R., Neri, R., Olmi, L., et al. 2005, A\&A, 434, 1039

Cesaroni, R., Galli, D., Lodato, G., Walmsley, C. M., \& Zhang, Q. 2007, in Protostars and Planets V, eds. B. Reipurth, D. Jewitt, \& K. Keil (University of Arizona Press), 197

Cesaroni, R., Galli, D., Neri, R., \& Walmsley, C.M. 2014, A\&A, 566, A73

Charnley, S. B., Tielens, A. G. G. M., \& Millar, T. J. 1992, ApJ, 399, L71

Comito, C., Schilke, P., Phillips, T. G., et al. 2005, ApJS, 156, 127

Curiel, S., Rodriguez, L. F., Canto, J., \& Torrelles, J. M. 1989, Rev. Mex. Astron. Astrofis., 17, 137

De Buizer, J. M. 2006, ApJ, 642, L57

de Wit, W. J., Hoare, M. G., Oudmaijer, R. D., et al. 2011, A\&A, 526, L5

Dent, W. R. F., Little, L. T., Kaifu, N., Ohishi, M., \& Suzuki, S. 1985a, A\&A, 146,375

Dent, W. R. F., Little, L. T., Sato, S., Ohishi, M., \& Yamashita, T. 1985b, MNRAS, 217, 217

Elia, D., Molinari, S., Fukui, Y., et al. 2013, ApJ, 772, 45

Forster, J. R., \& Caswell, J. L. 1989, A\&A, 213, 339

Forster, J. R., \& Caswell, J. L. 1999, A\&AS, 137, 43

Froebrich, D., Davis, C. J., Ioannidis, G., et al. 2011, MNRAS, 413, 480

Fuente, A., Neri, R., \& Caselli, P. 2005, A\&A, 444, 481

Fuente, A., Castro-Carrizo, A., Alonso-Albi, T., et al. 2009, A\&A, 507, 1475

Fuller, G. A., Zijlstra, A. A., \& Williams, S. J. 2001, ApJ, 555, L125

Gibb, A. G., Hoare, M. G., Little, L. T., \& Wright, M. C. H. 2003, MNRAS, 339, 1011

Gusdorf, A., Cabrit, S., Flower, D. R., \& Pineau Des Forêts, G. 2008a, A\&A, 482,809

Gusdorf, A., Pineau Des Forêts, G., Cabrit, S., \& Flower, D. R. 2008b, A\&A, 490, 695

Heaton, B. D., \& Little, L. T. 1988, A\&A, 195, 193

Hutawarakorn, B., \& Cohen, R. J. 1999, MNRAS, 303, 845

Kauffmann, J., \& Pillai, T. 2010, ApJ, 723, L7

Kauffmann, J., Pillai, T., \& Goldsmith, P. F. 2013, ApJ, 779, 185

Keto, E. 2007, ApJ, 666, 976
Kraus, S., Hofmann, K.-H., Menten, K. M., et al. 2010, Nature, 466, 339

Krumholz, M. R., \& McKee, C. F. 2008, Nature, 451, 1082

Krumholz, M. R., Klein, R. I., McKee, C. F., Offner, S. S. R., \& Cunningham, A. J. 2009, Science, 323, 754

Little, L. T., Dent, W. R. F., Heaton, B., Davies, S. R., \& White, G. J. 1985, MNRAS, 217, 227

Little, L. T., Kelly, M. L., \& Murphy, B. T. 1998, MNRAS, 294, 105

López-Sepulcre, A., Codella, C., Cesaroni, R., Marcelino, N., \& Walmsley, C. M. 2009, A\&A, 499, 811

López-Sepulcre, A., Walmsley, C. M., Cesaroni, R., et al. 2011, A\&A, 526, L2

MacLaren, I., Richardson, K. M., \& Wolfendale, A. W. 1988, ApJ, 333, 821

McMullin, J. P., Waters, B., Schiebel, D., Young, W., \& Golap, K. 2007, in Astronomical Data Analysis Software and Systems XVI, eds. R. A. Shaw, F. Hill, \& D. J. Bell, ASP Conf. Ser., 376, 127

Molinari, S., Swinyard, B., Bally, J., et al. 2010a, PASP, 122, 314

Molinari, S., Swinyard, B., Bally, J., et al. 2010b, A\&A, 518, L100

Möller, T., Bernst, I., Panoglou, D., et al. 2013, A\&A, 549, A21

Mookerjea, B., Casper, E., Mundy, L. G., \& Looney, L. W. 2007, ApJ, 659, 447

Mooney, T., Sievers, A., Mezger, P. G., et al. 1995, A\&A, 299, 869

Müller, H. S. P., Thorwirth, S., Roth, D. A., \& Winnewisser, G. 2001, A\&A, 370, L49

Nagasawa, M. 1987, Prog. Theor. Phys., 77, 635

Ossenkopf, V., \& Henning, T. 1994, A\&A, 291, 943

Peretto, N., Fuller, G. A., Duarte-Cabral, A., et al. 2013, A\&A, 555, A112

Pickett, H. M., Poynter, R. L., Cohen, E. A., et al. 1998, J. Quant. Spectr. Rad. Transf., 60, 883

Price, S. D., Egan, M. P., \& Shipman, R. F. 1999, in Astrophysics with Infrared Surveys: A Prelude to SIRTF, ASP Conf. Ser., 177, 394

Qiu, K., Zhang, Q., Menten, K. M., Liu, H. B., \& Tang, Y.-W. 2013, ApJ, 779, 182

Remijan, A., Sutton, E. C., Snyder, L. E., et al. 2004, ApJ, 606, 917

Sánchez-Monge, Á., Palau, A., Estalella, R., et al. 2010, ApJ, 721, L107

Sánchez-Monge, Á., Beltrán, M. T., Cesaroni, R., et al. 2013a, A\&A, 550, A21

Sánchez-Monge, Á., Cesaroni, R., Beltrán, M. T., et al. 2013b, A\&A, 552, L10

Sánchez-Monge, Á., Palau, A., Fontani, F., et al. 2013c, MNRAS, 432, 3288

Sánchez-Monge, Á., López-Sepulcre, A., Cesaroni, R., et al. 2013d, A\&A, 557, A94

Schilke, P., Benford, D. J., Hunter, T. R., Lis, D. C., \& Phillips, T. G. 2001, ApJS, 132,281

Schreyer, K., Henning, T., van der Tak, F. F. S., Boonman, A. M. S., \& van Dishoeck, E. F. 2002, A\&A, 394, 561

Schuller, F., Menten, K. M., Contreras, Y., et al. 2009, A\&A, 504, 415

Simon, M., Dutrey, A., \& Guilloteau, S. 2000, ApJ, 545, 1034

Sugiyama, K., Fujisawa, K., Doi, A., et al. 2008, PASJ, 60, 23

Surcis, G., Vlemmings, W. H. T., van Langevelde, H. J., \& Hutawarakorn Kramer, B. 2012, A\&A, 541, A47

Tan, J. C., Beltrán, M. T., Caselli, P., et al. 2014, Protostars and Planets VI (University of Arizona Press), accepted [arXiv: 1402 .0919]

Vallée, J. P., \& Bastien, P. 2000, ApJ, 530, 806

Velusamy, T., Marsh, K. A., Beichman, C. A., Backus, C. R., \& Thompson, T. J. 2008, AJ, 136, 197

Vlemmings, W. H. T. 2008, A\&A, 484, 773

Walther, D. M., Aspin, C., \& McLean, I. S. 1990, ApJ, 356, 544

Wang, K.-S., Kuan, Y.-J., Liu, S.-Y., \& Charnley, S. B. 2010, ApJ, 713, 1192

Wang, K., Zhang, Q., Wu, Y., \& Zhang, H. 2011, ApJ, 735, 64

Wang, K.-S., van der Tak, F. F. S., \& Hogerheijde, M. R. 2012, A\&A, 543, A 22

Wang, K.-S., Bourke, T. L., Hogerheijde, M. R., et al. 2013, A\&A, 558, A69

Wang, K., Zhang, Q., Testi, L., et al. 2014, MNRAS, 439, 3275

Williams, J. P., de Geus, E. J., \& Blitz, L. 1994, ApJ, 428, 693

Wilson, T. L., \& Rood, R. 1994, ARA\&A, 32, 191

Wright, E. L., Eisenhardt, P. R. M., Mainzer, A. K., et al. 2010, AJ, 140, 1868

Zernickel, A., Schilke, P., Schmiedeke, A., et al. 2012, A\&A, 546, A87

Zhang, B., Zheng, X. W., Reid, M. J., et al. 2009, ApJ, 693, 419

Zhang, Y., Tan, J. C., De Buizer, J. M., et al. 2013, ApJ, 767, 58

Zinnecker, H., \& Yorke, H. W. 2007, ARA\&A, 45, 481 

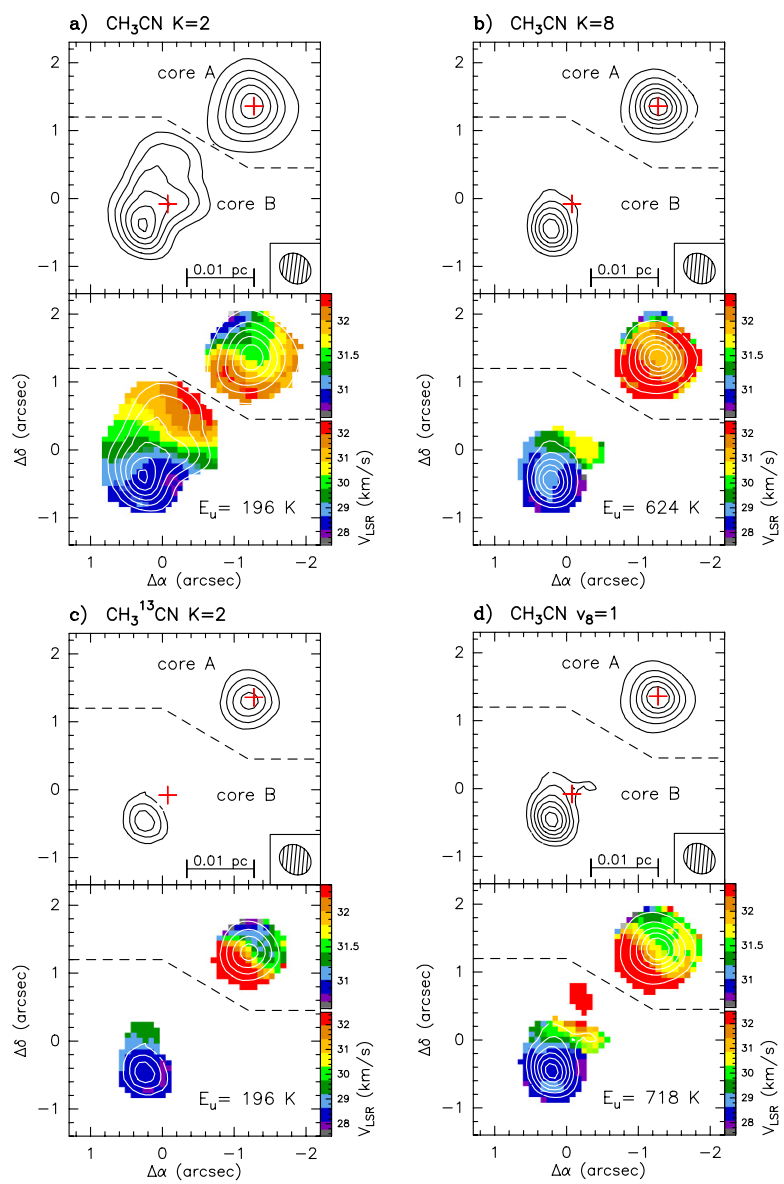

Fig. A.1. Maps of the integrated intensity (upper panels) and velocity field (bottom panels) of a) $\mathrm{CH}_{3} \mathrm{CN} K=2$ at $349.426 \mathrm{GHz}$; b) $\mathrm{CH}_{3} \mathrm{CN}$ $K=8$ at $349.024 \mathrm{GHz}$; c) $\mathrm{CH}_{3}^{13} \mathrm{CN} K=2$ at $349.254 \mathrm{GHz}$; and d) $\mathrm{CH}_{3} \mathrm{CN} v_{8}=1 \mathrm{~K}, l=3,1$ at $350.552 \mathrm{GHz}$ towards cores A and B. For the different panels, the starting (and increasing contour levels in terms of $\sigma$, in Jy beam $\left.{ }^{-1} \mathrm{~km} \mathrm{~s}^{-1}\right)$ are $5 \sigma(10 \sigma$, with $\sigma=0.15), 5 \sigma(10 \sigma$, with $\sigma=0.15), 3 \sigma(3 \sigma$, with $\sigma=0.10)$, and $5 \sigma(10 \sigma$, with $\sigma=0.10)$ for core A; and $5 \sigma(5 \sigma$, with $\sigma=0.09), 4 \sigma(4 \sigma$, with $\sigma=0.09), 2 \sigma(2 \sigma$, with $\sigma=0.06)$, and $3 \sigma(3 \sigma$, with $\sigma=0.06)$ for core B. See details and description of symbols in Fig. 4.

\section{Appendix A: Hot core intensity and velocity maps}

In Figs. A.1 and A.2, we present the zeroth (integrated intensity) and first (velocity field) moment maps of various hot-core tracers detected towards cores A and B. In Sect. 3.2.2, we describe how the moments have been obtained, while the symbols shown in these two figures are described in the caption of Fig. 4.

\section{Appendix $\mathrm{B}: \mathrm{CH}_{3} \mathrm{OH}$ and $\mathrm{CH}_{3} \mathrm{CN}$ myXCLASS fits}

In Figs. B.1-B.4 we show the spectra of the bulge of methanol $\left(\mathrm{CH}_{3} \mathrm{OH}\right.$, black line in the top panels) and methyl cyanide $\left(\mathrm{CH}_{3} \mathrm{CN}\right.$, black line in the bottom panels) lines observed toward cores A, B, C and D. For this last core, the methyl cyanide lines are too weak and cannot be fitted. The observed spectra have been fitted (red lines) using myXCLASS (see Sect. 4.1). The procedure searches, by minimizing the $\chi^{2}$, for the best fit of five parameters: size (in arcsec), temperature (in K), column density (in $\mathrm{cm}^{-2}$ ), linewidth (in $\mathrm{km} \mathrm{s}^{-1}$ ) and LSR velocity (in $\mathrm{km} \mathrm{s}^{-1}$ ). The blue lines in the small panels show the $\chi^{2}$ of the fits for different values of the parameters. In Table 3, we list the values of the parameters obtained in the best fits. We note that we have
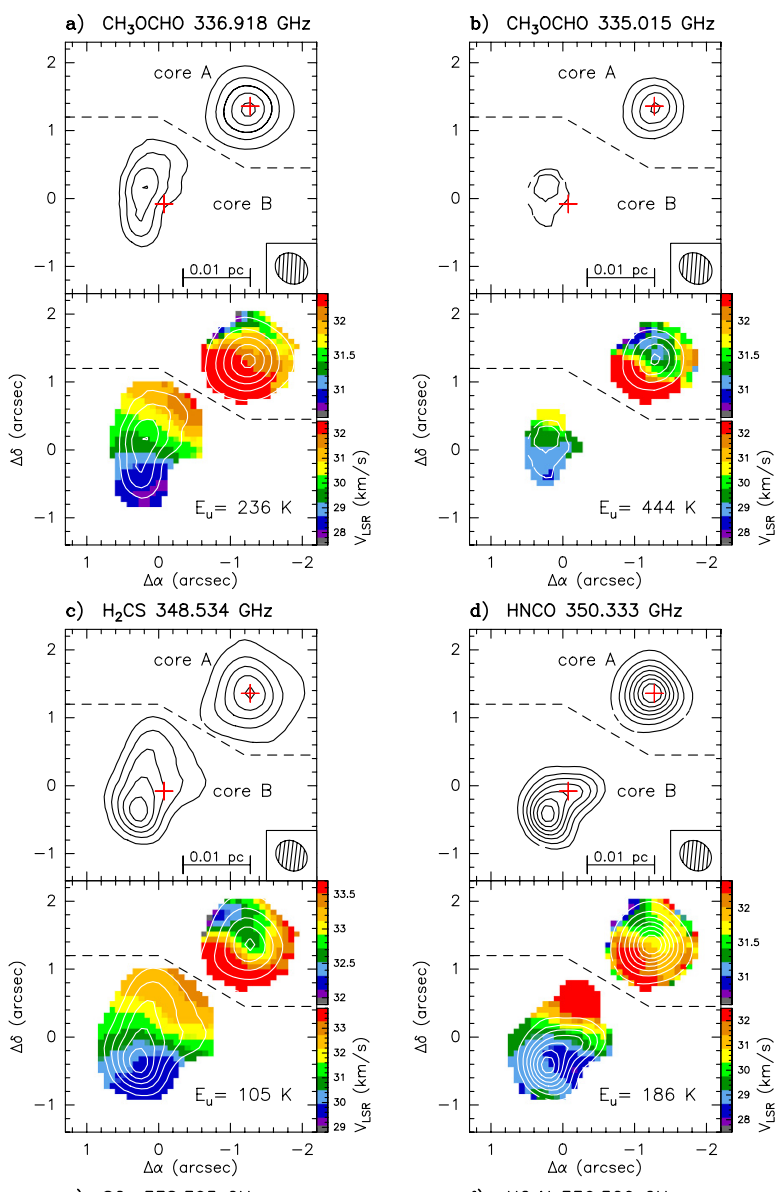

d) $\mathrm{HNCO} 350.333 \mathrm{GHz}$
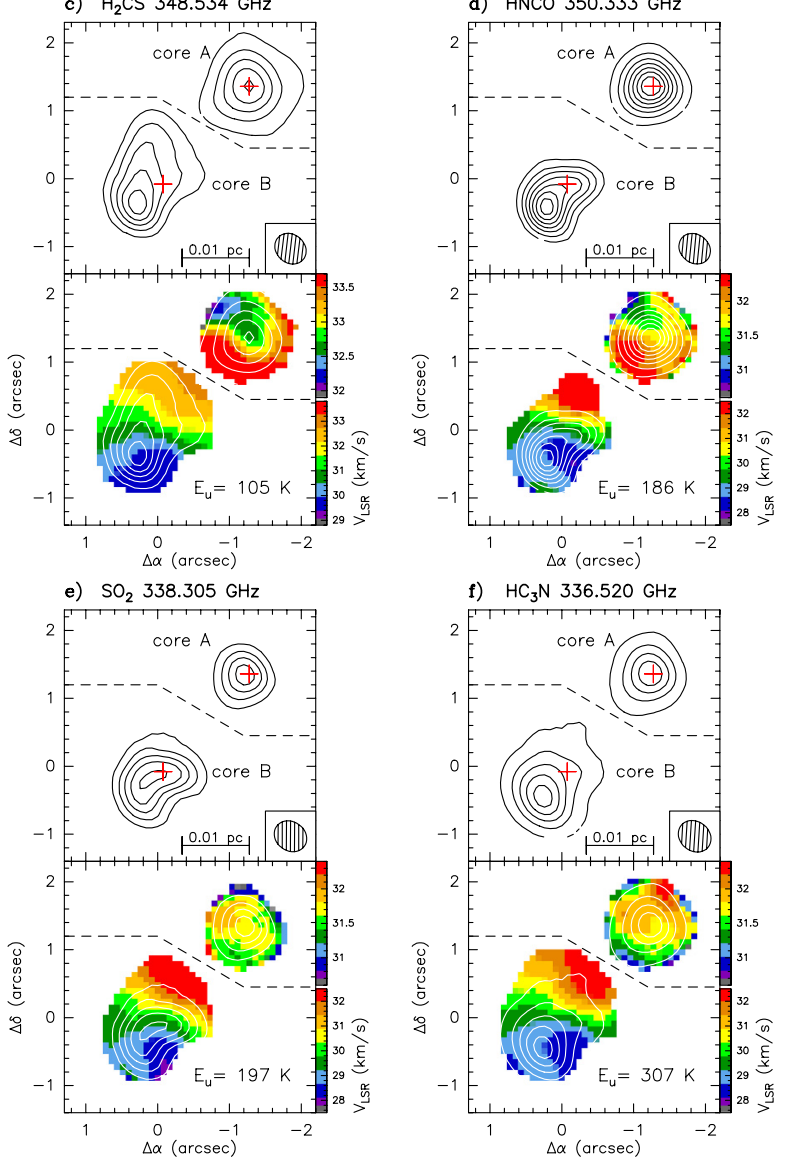

Fig. A.2. Maps of the integrated intensity (upper panels) and velocity field (bottom panels) of a) $\mathrm{CH}_{3} \mathrm{OCHO}\left(26_{6,20}-25_{6,19}\right)$; b) $\mathrm{CH}_{3} \mathrm{OCHO}\left(27_{7,21}-26_{7,20}\right)$; c) $\mathrm{H}_{2} \mathrm{CS}\left(10_{1,9}-9_{1,8}\right)$, d) $\mathrm{HNCO}\left(16_{1,16}\right.$ $\left.15_{1,15}\right)$; e) $\mathrm{SO}_{2}\left(18_{4,14}-18_{3,15}\right)$; and f) $\mathrm{HC}_{3} \mathrm{~N}(37-36)$ towards cores $\mathrm{A}$ and $\mathrm{B}$. For the different panels, the starting (and increasing contour levels in terms of $\sigma$, in Jy beam $\left.{ }^{-1} \mathrm{~km} \mathrm{~s}^{-1}\right)$ are $5 \sigma(10 \sigma$, with $\sigma=0.06), 3 \sigma(3 \sigma$, with $\sigma=0.04), 5 \sigma(10 \sigma$, with $\sigma=0.16), 5 \sigma(10 \sigma$, with $\sigma=0.10), 5 \sigma$ $(5 \sigma$, with $\sigma=0.20)$, and $5 \sigma(10 \sigma$, with $\sigma=0.11)$ for core A; and $3 \sigma$

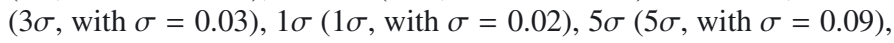
$5 \sigma(5 \sigma$, with $\sigma=0.06), 5 \sigma(5 \sigma$, with $\sigma=0.10)$, and $5 \sigma(10 \sigma$, with $\sigma=0.07$ ) for core B. See details and description of symbols in Fig. 4.

simultaneously fitted optically thick and thin transitions (e.g., ground state, vibrationally excited and isotopologues of $\mathrm{CH}_{3} \mathrm{CN}$ and $\mathrm{CH}_{3} \mathrm{OH}$ ), thus avoiding possible degeneracies (see Zernickel et al. 2012) between the parameters of the fit (e.g., column density and size). 

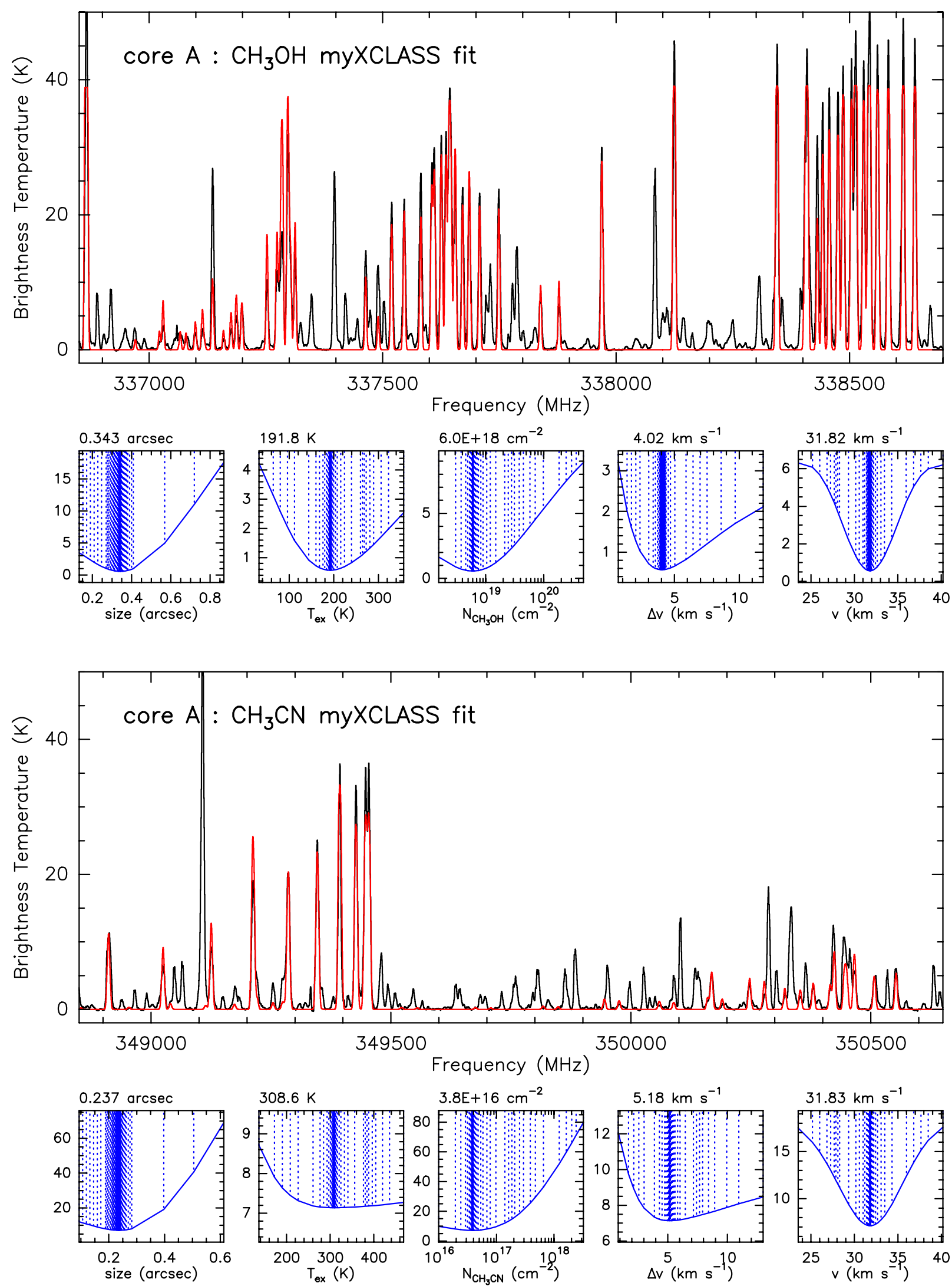

Fig. B.1. Large panels: observed $\mathrm{CH}_{3} \mathrm{OH}$ (top panel) and $\mathrm{CH}_{3} \mathrm{CN}$ (bottom panel) lines toward core A in G35.20-0.74 N. The red line shows the best fitted obtained by myXCLASS (see Sect. 4.1). The fit in the top panel includes ground state $\mathrm{CH}_{3} \mathrm{OH}$ and ${ }^{13} \mathrm{CH}_{3} \mathrm{OH}$ and torsionally excited $\mathrm{CH}_{3} \mathrm{OH}$ lines, while the fit in the bottom panel includes ground state $\mathrm{CH}_{3} \mathrm{CN}$ and $\mathrm{CH}_{3}^{13} \mathrm{CN}$ and vibrationally excited $\mathrm{CH}_{3} \mathrm{CN}$ lines in the frequency ranges shown in the panels. Small panels: $\chi^{2}$ values obtained from the fits minimization. From left to right the panels show the size, the temperature, the column density, the linewidth and the LSR velocity. 

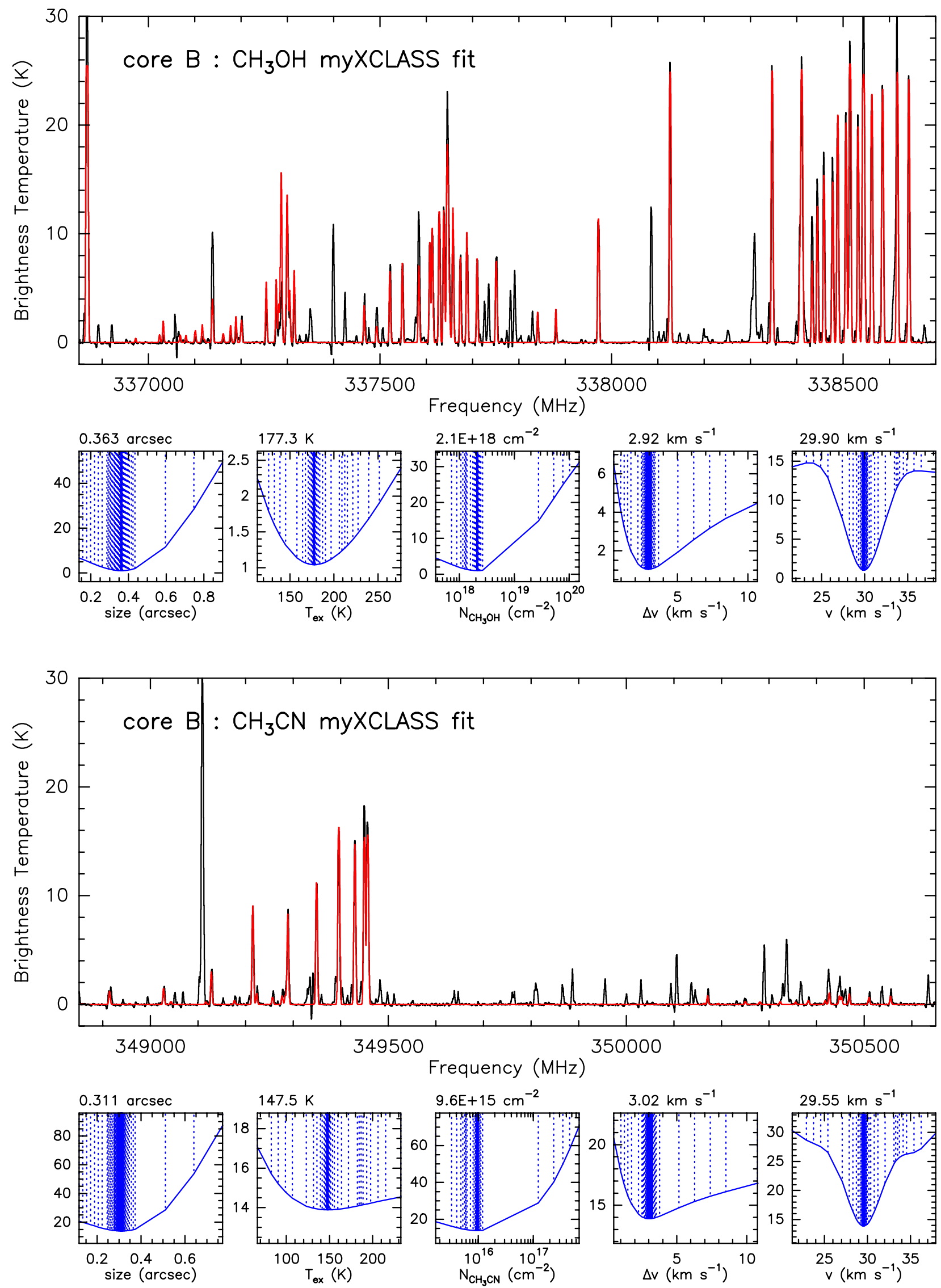

Fig. B.2. Same as Fig. B.1 for core B. 

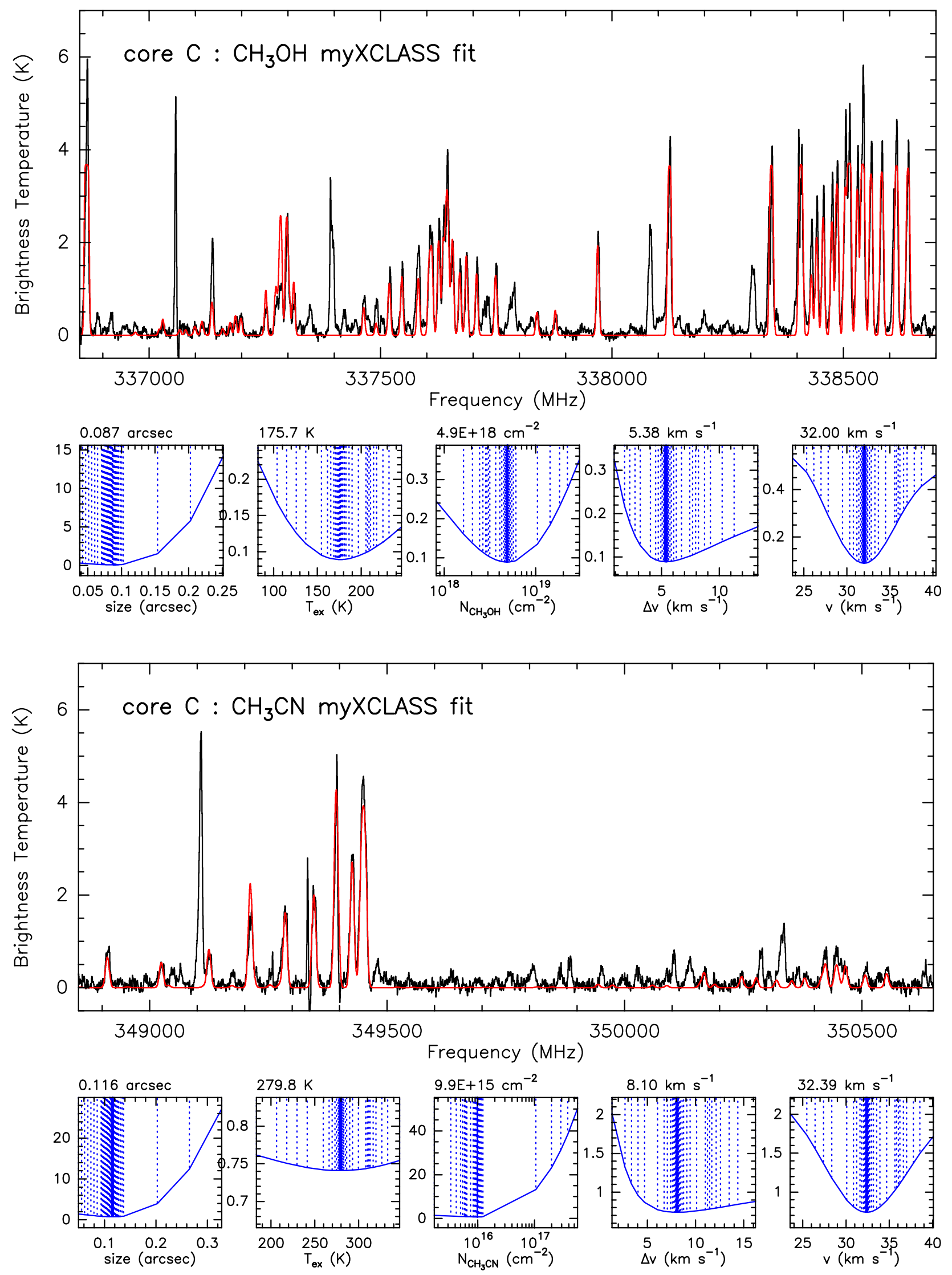

Fig. B.3. Same as Fig. B.1 for core C. 
A\&A 569, A11 (2014)
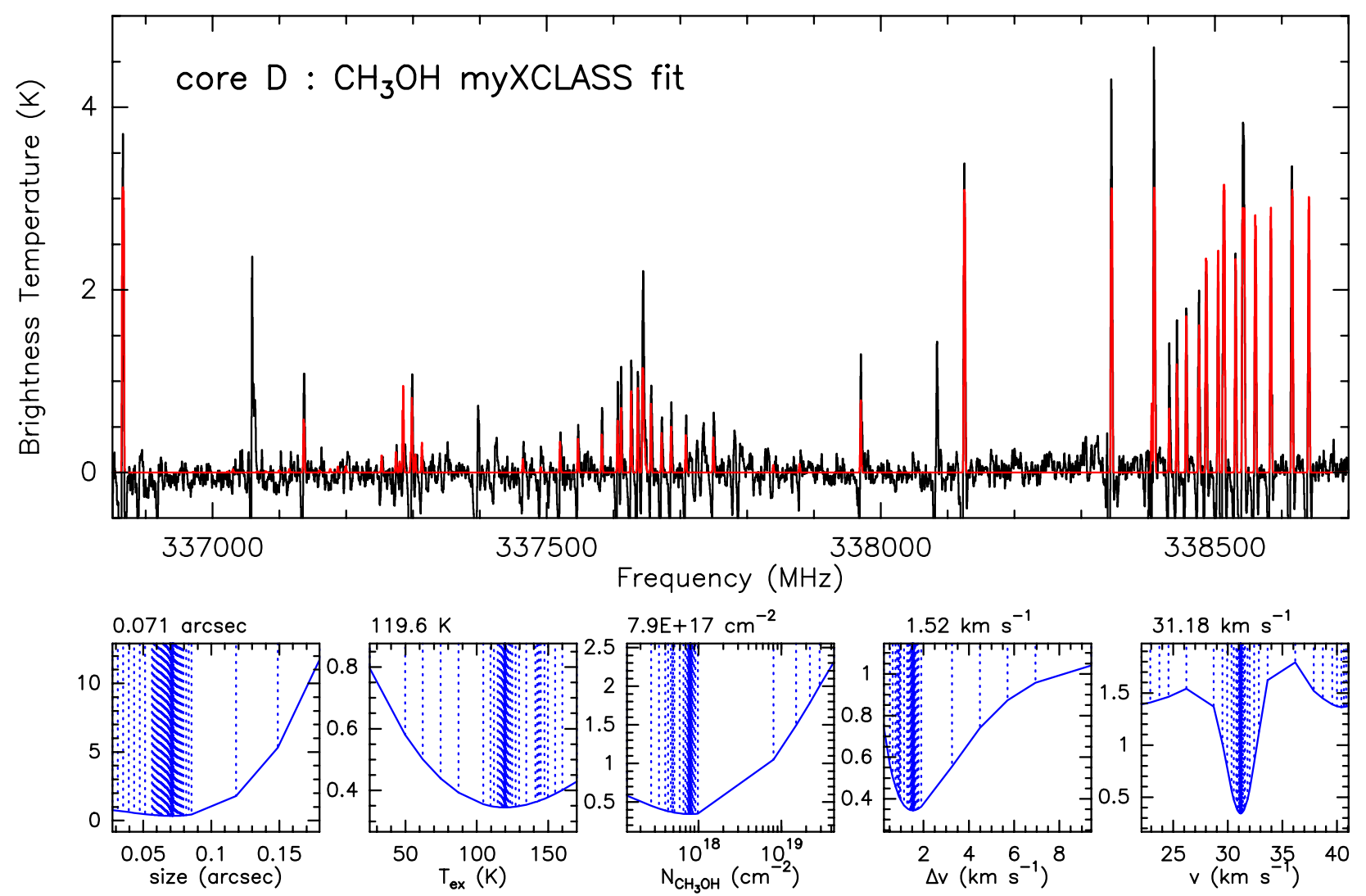

Fig. B.4. Same as Fig. B.1 for core D, and only for $\mathrm{CH}_{3} \mathrm{OH}$ lines. 